\title{
TECHNIQUES TO CHARACTERIZE VAPOR CELL PERFORMANCE FOR A NUCLEAR-MAGNETIC-RESONANCE GYROSCOPE
}

\author{
A Thesis \\ presented to \\ the Faculty of California Polytechnic State University, \\ San Luis Obispo
}

\author{
In Partial Fulfillment \\ of the Requirements for the Degree \\ Master of Science in Aerospace Engineering
}

by

James Julian Mirijanian

May 2012 
(C) 2012

James Julian Mirijanian

ALL RIGHTS RESERVED 


\section{COMMITTEE MEMBERSHIP}

TITLE:

Techniques to Characterize Vapor Cell Performance for a Nuclear-Magnetic-Resonance Gyroscope
AUTHOR:
James Julian Mirijanian

DATE SUBMITTED: $\quad$ May 2012

COMMITTEE CHAIR: $\quad$ Dr. Eric Mehiel, Cal Poly Aerospace Engineering

COMMITTEE MEMBER: Dr. Kira Abercromby, Cal Poly Aerospace Engineering

COMMITTEE MEMBER: Dr. Jordi Puig-Suari, Cal Poly Aerospace Engineering

COMMITTEE MEMBER: Dr. Michael S. Larsen, Northrop Grumman, NSD 


\author{
ABSTRACT \\ Techniques to Characterize Vapor Cell Performance for a \\ Nuclear-Magnetic-Resonance Gyroscope \\ James Julian Mirijanian
}

Research was performed to improve the procedures for testing performance parameters of vapor cells for a nuclear-magnetic-resonance gyroscope. In addition to summarizing the theoretical infrastructure of the technology, this research resulted in the development and successful implementation of new techniques to characterize gyro cell performance.

One of the most important parameters to measure for gyro performance is the longitudinal spin lifetime of polarized xenon atoms in the vapor cell. The newly implemented technique for measuring these lifetimes matches results from the industry standard method to within 3.5\% error while reducing the average testing time by $76 \%$ and increasing data resolution by $54 \%$. The vapor cell test methods were appended with new software to expedite the analysis of test data and to investigate more subtle details of the results; one of the two isotopes of xenon in the cells tends to exhibit troublesome second-order effects during these tests due to electric-quadrupole coupling, but now the added analysis capabilities can accurately extract relevant results from such data with no extra effort. Some extraneous lifetime measurement techniques were explored with less substantial results, but they provided useful insight into the complex workings of the gyro cell test system.

New criteria were established to define the signal to noise ratio on a consistent basis from cell to cell across various parameters such as cell volume, temperature, and vapor pressure. A technique for measuring gas pressures inside the sealed cells helped link cell performance to cell development processes. This led to informed decisions on filling and sealing methods that consistently yielded cells with better performance in the last few months of this work. When this research began, cells with xenon lifetimes over ten seconds were rare in our lab; by the end, anything under 30 seconds was a disappointment. Not only did the test procedures improve, but so did the parameters being tested, and quite significantly at that. At the same time, many new avenues for continued progress have been opened; the work presented here, while instrumental, is only the beginning. 


\section{ACKNOWLEDGMENTS}

Three years prior to the completion of this work, I had never suspected that pursuing a degree in Aerospace Engineering would land me a job assembling lasers and learning atomic physics. It amazes me that the path of a career can be determined by a momentary impulse.

Between the time that I first got accepted into the graduate program at Cal Poly and the time I actually found a thesis topic to work on, I attended a great course in quantum mechanics led by Dr. Thomas Gutierrez of the Cal Poly physics department. Remembering that my thesis advisor, Dr. Eric Mehiel, had studied physics as an undergraduate, I decided on a whim to ask if he knew anything about applying quantum mechanics to aerospace controls systems (his primary field of work). To my surprise and great fortune, he told me he had recently learned of a project along those lines being pursued outside the university by his associates on another project.

A few months later, I had met Doug Meyer and Charles Volk and was working as a summer intern in their department to learn everything I could about their new development - a small, low-power, highly sensitive nuclear-magnetic-resonance gyroscope. In my few short months there that summer, I think I learned more than in the entire year prior, which is saying quite a lot. Because of the invaluable and unwavering mentorship offered by Dr. Michael S. Larsen, Mike Bulatowicz, Jim Pavell, and the entire staff of great minds all willing to aid and teach at any time, by the end of my internship there I had become familiar enough with the technology and the science behind it to construct a thesis topic that would actually benefit the program.

Years later (at least a full year more than what seems reasonable), now I've become so comfortable with the apparatus that I sometimes forget how intricate and impressive the technology is. I'm certain I haven't even traversed the tip of the iceberg yet, but the progress I've made in my own understanding is substantial, and I have everyone mentioned above to thank for that.

Of course, I never would have been in Dr. Mehiel's office, asking him that question, had it not been for the unlimited opportunities provided by my loving family. My mother and father, Kathy Cotter and Craig Mirijanian, worked relentlessly to motivate me to do well, providing a great education and many luxuries at home that I feel blessed to have experienced. My older brother, Deran, has always been an inspiration and a great support. To neglect thanking my family would nullify this work in my own eyes.

After nearly twenty years of focusing primarily on academia, this is one of the last paragraphs I might ever write for what is still technically a "school paper". As such, I can't help erroneously thinking that this dissertation somehow stands for my entire career as a student. I've tried to make it accessible to people with very little prior knowledge on the subject, as was the case for me when I started this work. If I can spark in others even a fraction of the wonder that was evoked in me by those who led me here, I will have accomplished my goal. Thank you all and I hope you enjoy the read! 


\section{TABLE OF CONTENTS}

\section{Page}

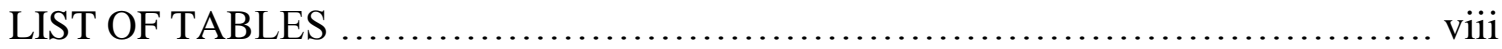

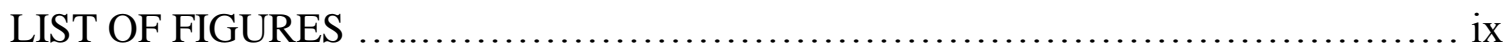

LIST OF EQUATIONS ................................................. xiii

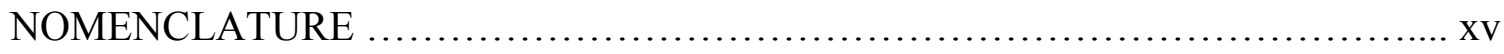

SUBSRCIPTS AND SUPERSCRIPTS .................................... xvi

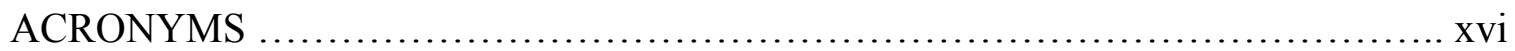

I. Introduction to Navigation, Inertial Measurement, and Gyroscopes $\ldots \ldots \ldots \ldots \ldots \ldots \ldots 1$

$\mathbf{A}_{\mathbf{I}}$. History and Basic Operation of Gyroscopes ................................ 3

B $_{\mathbf{I}}$ How the NMR Gyroscope Cell Test Apparatus Works ........................ 7

II. How the NMR Gyroscope Cell Test Apparatus Really Works .................... 13

$\mathbf{A}_{\text {II }}$. Cornerstone Physical Principles in Achieving Magnetic Resonance ............ 13

$\mathbf{1}_{\text {AII }}$ Particle Spin and Larmor Precession .................................. 13

$\boldsymbol{2}_{\text {AII. }}$ Relevant Properties of Electromagnetic Radiation ....................... 17

$\mathbf{3}_{\text {AII }}$ Quantized Atomic Energy Transitions and Optical Pumping ................ 22

äAII. Spin-Exchange Optical Pumping ................................. 34

$\mathbf{4}_{\text {AII }}$ Alkali Precession and Magnetometer Signal .......................... 36

$\mathbf{a}_{4 \text { AII. Faraday Detection } \ldots \ldots \ldots \ldots \ldots \ldots \ldots \ldots \ldots \ldots \ldots \ldots \ldots \ldots \ldots \ldots \ldots} 43$

$\mathbf{5}_{\mathrm{AII}}$. Xenon Precession and the NMR Gyroscope Signal ...................... 46

$\mathbf{B}_{\text {II. }}$ Lifetimes of Nuclear Spin States ..................................... 54

1 $_{\text {BII. }}$ The Major Influences on Longitudinal Spin Lifetimes .................... 56

III. Techniques for Measuring Spin Lifetimes for NMR ......................... 60

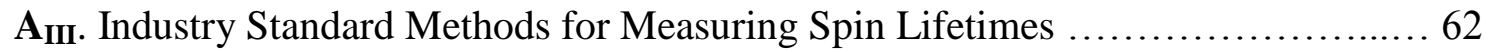


$\mathbf{1}_{\text {AIII. }}$ Measuring T2 Using the Free Induction Decay Method $\ldots \ldots \ldots \ldots \ldots \ldots \ldots . \ldots 3$

a 1 AIII. The ${ }^{131} \mathrm{Xe}$ Isotope and Electric Quadrupole Coupling ............... 69

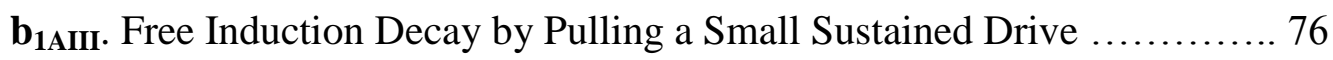

$\boldsymbol{2}_{\text {AIII. }}$ Measuring T1 Using the Delayed Pulse Method $\ldots \ldots \ldots \ldots \ldots \ldots \ldots \ldots \ldots . \ldots 2$

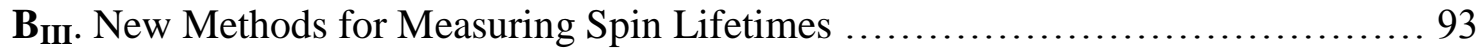

1

a $\mathbf{a}_{\text {1BIII. }}$ Shape Inconsistencies in the Growth Method ....................... 98

b $_{1 \text { BIII. }}$ Offsetting the Drive Frequency to Eliminate Beating .............. 100

$\mathbf{2}_{\text {BIII. }}$ Measuring T1 Using the Flipped Polarization Method ................... 107

a $\mathbf{2 B I I I}$. The Quarter-Wave Plate Approach ............................ 109

b

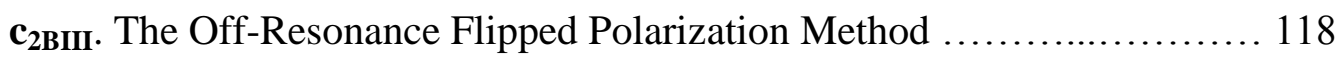

$\mathbf{d}_{\text {2BIII. }}$ Sensitivities in Flipped Polarization Results ..................... 125

3 $_{\text {BIII. }}$ Measuring T2 and T1 Simultaneously Using the Pump Growth Method ... 136

IV. Conclusions ....................................................... 149

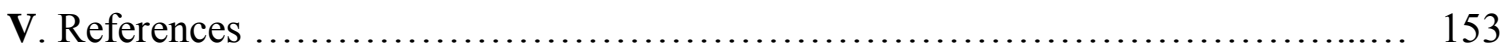




\section{LIST OF TABLES}

Table

Page

1. Solution Parameters Used to Fit ${ }^{131}$ Xe Free Induction Decay, Extreme Case .... 72

2. Values Used to Fit ${ }^{131}$ Xe Decay Profile with Improper Start Time .............. 74

3. Values Used to Fit ${ }^{131}$ Xe Decay Profile, Typical Case ........................ 75

4. Comparing Solution Values for Sustained Drive vs. Pulse for ${ }^{131}$ Xe Decay ..... 78

5. Solution Parameters Used to Fit Delayed Pulse Profile ....................... 88

6. Comparing Solution Values for ${ }^{131}$ Xe Delayed Pulse Analysis Approaches ...... 92

7. Comparison between Growth and Decay Methods for Measuring T2 ......... 103

8. Solution Parameters Used to Fit Flipped Polarization Profile ................. 113

9. Comparing Flipped Polarization Approaches to the Delayed Pulse Test .......... 121

10. Comparing T1 Metrics for the Flipped Polarization Test .................. 122

11. Solutions for Inconsistent ${ }^{131}$ Xe Flipped Polarization Profiles ................ 123

12. Comparing T1 Metrics for the ${ }^{131}$ Xe Flipped Polarization Test ................ 124

13. Compiled Results Comparing Flipped Polarization to Delayed Pulse .......... 133 


\section{LIST OF FIGURES}

Figure

Page

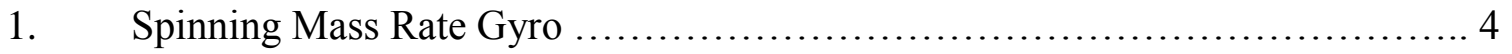

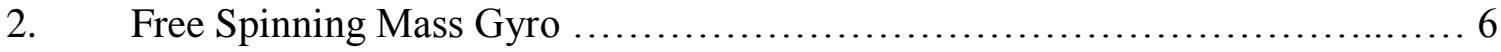

3. Classical versus Atomic Angular Momentum ............................ 9

4. Simplified Schematic of the Vapor Cell Test Apparatus ....................... 11

5. Larmor Precession of a Subatomic Particle .............................. 15

6. Normal Atomic Distribution versus Magnetic Resonance Distribution .......... 17

7. Polarization of Light and Photon Angular Momentum ...................... 19

8. Photon Polarization in Circular and Linear-Orthogonal Bases ................ 20

9. Useful Optical Tools for Atomic Applications .......................... 21

10. Single Valence Electron Outside the Ion Core of an Alkali Atom .............. 23

11. Partial Energy Level Structure of a Cesium Atom ......................... 25

12. Light Transmission Intensity Profiles Showing Cesium Absorption Lines ...... 29

13. Cesium Absorption Width as a Measure of Buffer Gas Pressure ............... 31

14. The Effect of Optical Pumping as Seen in Absorption Profiles ................ 33

15. Alkali Response to a Transverse DC Field ............................. 38

16. Component Description of the Alkali Stimulation Process ................... 39

17. The Atomic Magnetometer Carrier Signal ............................. 41

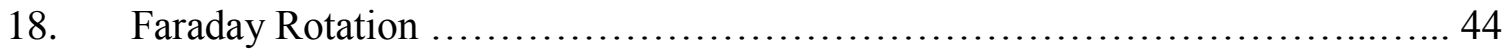

19. Faraday Effect Dependence on Alkali Precession ........................ 45

20. Faraday Detection Setup for the Atomic Magnetometer .................... 46

21. NMR Gyro Basic Concept of Operation ............................... 48

22. NMR Gyro ARW Relative to Cell Performance Parameters ................... 52

23. Effect of Temperature on Xenon Longitudinal Spin Lifetimes, Extreme Case ... 57

24. Effect of Temperature on Xenon Longitudinal Spin Lifetimes, Typical Case .... 58 


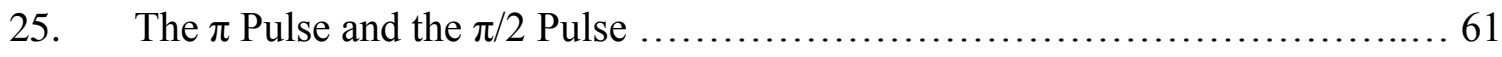

26. Determining the Appropriate Amplitude for a $\pi$ Pulse ..................... 61

27. Raw Data from a Free Induction Decay T2 Test for ${ }^{129} \mathrm{Xe} \ldots \ldots \ldots \ldots \ldots \ldots \ldots . \ldots 6 . \ldots . \ldots . \ldots$

28. Frequency-Spectrum Data from Free Induction Decay T2 Test .............. 65

29. Signal Profile from the FFT Amplitude of the Precession Frequency ........... 66

30. Signal Profile with Fit Function and Half-Life .......................... 68

31. Data from a Free Induction Decay T2 Test for ${ }^{131} \mathrm{Xe}$, Extreme Case ............ 69

32. FFT with Relevant Parameters Labeled to Describe the Quadrupole Effects ... 70

33. Fitted Signal Profile for ${ }^{131}$ Xe Free Induction Decay, Extreme Case ............ 73

34. Consequences of Choosing an Improper Start Time for Data Analysis .......... 74

35. Data and Fitted Profile for ${ }^{131}$ Xe Free Induction Decay, Typical Case .......... 75

36. Comparing the Pulse to a Small Sustained Drive for Stimulation of ${ }^{129} \mathrm{Xe} \ldots \ldots .77$

37. Comparing the Pulse to a Small Sustained Drive for Stimulation of ${ }^{131} \mathrm{Xe} \ldots \ldots .78$

38. Effect of Using a Drive Frequency Higher than the Resonance ............... 80

39. Effect of Using a Drive Frequency Lower than the Resonance ............... 81

40. Conceptual Physics behind the Delayed Pulse Method for Measuring T1 ....... 83

41. Sample Data from a Delayed Pulse T1 Test $\ldots \ldots \ldots \ldots \ldots \ldots \ldots \ldots \ldots \ldots \ldots \ldots$

42. Modified Delayed Pulse Profiles for Extracting a T1 Value ................. 86

43. Fitted Profile for Delayed Pulse T1 Test, Extreme Case .................... 87

44. Fitted Profile for Delayed Pulse T1 Test, Typical Case ..................... 89

45. Comparison of T1 Metrics based on Sensitivity to Data Density .............. 90

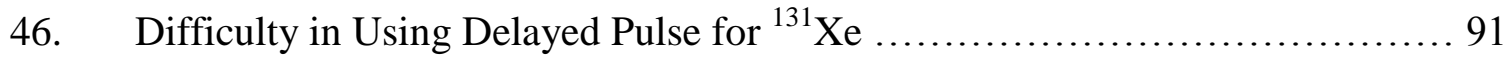

47. Comparison of Data Collection Methods for ${ }^{131}$ Xe Delayed Pulse Tests ......... 92

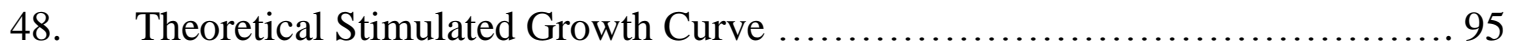

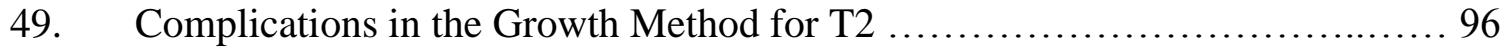

50. Exceptionally Well Behaved T2 Growth Data ........................... 97 
Figure

51. Data from Off-Resonance-Stimulated Growth Test ..................... 100

52. Off-Resonance Growth Using an Arbitrary Window Size ................... 101

53. Frequency-Specific Signal Profiles Using the Appropriate Window Size ..... 102

54. Fitted Growth Profile Obtained by Combining the Two Raw Profiles .......... 103

55. Growth Results Dependence on Drive Frequency Offset .................. 105

56. Quadrupole Effects Still Present in Off-Resonance ${ }^{131}$ Xe Growth Test ........ 106

57. Using a Quarter-Wave Plate to Prepare the Flipped Polarization T1 Test ..... 108

58. Physics behind the Plate Approach to the Flipped Polarization Method ........ 110

59. Example Data from a Plate-Approach Flipped Polarization Test ............. 111

60. Delayed Pulse Results by which to Assess the Flipped Polarization Method ... 112

61. Fitted Plate-Approach Flipped Polarization Profile ......................... 114

62. Physics behind the Pulse Approach to the Flipped Polarization Method ....... 115

63. Example Data Profile for Pulse Approach to Flipped Polarization Method ..... 116

64. Low-Drive-Amplitude Example Data Profiles for Both Approaches .......... 117

65. Delayed Pulse Results by which to Assess the Off-Resonance Test ............. 119

66. Data from Both Approaches to Flipped Polarization ....................... 120

67. Fitted Profiles for Both Approaches Driving Off Resonance ................ 121

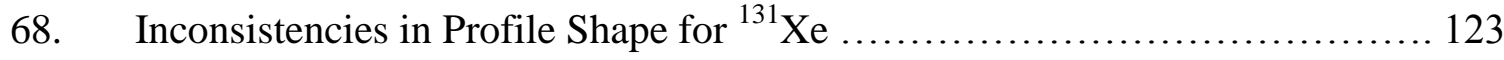

69. Effect of Off-Resonance Drive on Quadrupole-Coupled Populations ........... 124

70. Flipped Polarization Profile Shape Sensitivity to Pulse Strength .............. 126

71. Flipped Polarization Solution Parameter Sensitivity to Pulse Strength ......... 127

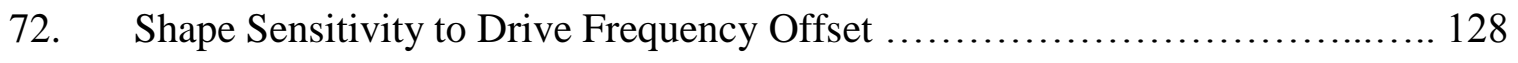

73. Inexplicable Shape Defects in On-Resonance Profile ...................... 129

74. Solution Parameter Sensitivity to Drive Frequency Offset ................... 130

75. Sensitivities to Window Size Errors during Analysis ..................... 132

76. Physics behind the Pump Growth Method ............................. 137 
Figure Page

77. Theoretical Pump Growth Curves Varying the Ratio of T2:T1 .............. 138

78. Example Data from an On-Resonance Pump Growth Test .................... 139

79. Industry-Standard T1 and T2 Tests by which to Assess the Pump Growth ..... 140

80. Fitted Profile for the Pump Growth Test ............................... 141

81. Pump Growth Sensitivities to Drive Parameters, Course Range .............. 142

82. Pump Growth Sensitivities to Drive Parameters, Fine Range ................. 143

83. Example Data from an Off-Resonance Pump Growth Test ................... 145

84. Off-Resonance Pump Growth Shape Sensitivity to Drive Amplitude .......... 146

85. Pump Growth Solution Parameter Sensitivity to Drive Amplitude ............. 147 


\section{LIST OF EQUATIONS}

Equation

Page

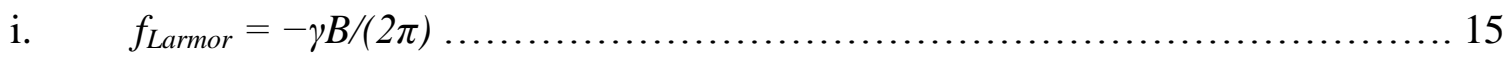

the natural Larmor frequency for a particle in a magnetic field offset from its spin

ii. $\quad E=h v=h c / \lambda$

the energy of a photon characterized by the wavelength and frequency of the EM field

1. $y=A e^{\wedge}(-t / \tau)+v$ 66

the fit function for analyzing free induction decay $T 2$ test data

2. $\tau=T_{1 / 2} / L N(2)$

the relationship between the lifetime and half-life for a given atomic transition

3. $y=A_{C} \sin \left(\omega_{C} t\right) e^{\wedge}\left(-t / T 2_{C}\right)+A_{Q}\left[\sin \left(\omega_{L} t\right)+\sin \left(\omega_{R} t\right)\right] e^{\wedge}\left(-t / T 2_{Q}\right)$

the theoretical description of the raw signal generated in a quadrupole-coupled T2 test

4. $\quad y=\left|A_{C} e^{\wedge}\left(-t / T 2_{C}\right)+2 A_{Q} \cos (\delta \omega t) e^{\wedge}\left(-t / T 2_{Q}\right)\right|+v$

the fit function for quadrupole-coupled free induction decay T2 test data

5. $y=A\left[1-e^{\wedge}(-t / \tau)\right]+v$ 85

the fit function for stimulated growth T2 test data

6. $y=\left|A e^{\wedge}(-t / \tau)-V\right|+v$

the fit function for delayed pulse and off-resonance flipped polarization T1 test data 
Equation

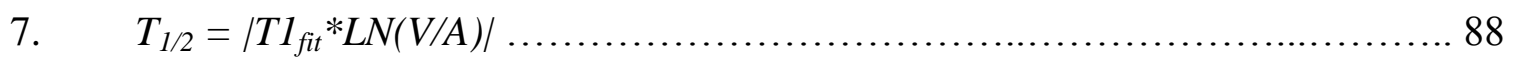

the relationship between the apparent half-life and the fit solution parameters for T1 tests

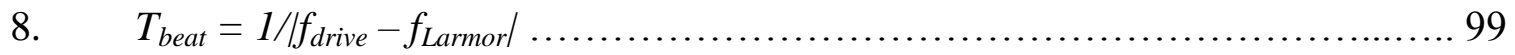

the beat period which defines window size for analyzing off-resonance-stimulated tests

9. $y=A^{*}\left|e^{\wedge}(-t / T 1)-V\right| *\left[1-e^{\wedge}(-t / T 2)\right]+v$

the fit function for on-resonance flipped polarization tests using the $\lambda / 4$ plate approach

10. $y=A *[1-\exp (-t / T 2)] *[1-\exp (-t / T 1)]+v$

the fit function for pump growth T1 and T2 test 


\section{NOMENCLATURE}

\begin{tabular}{|c|c|}
\hline${ }^{129} \mathrm{Xe}$ & The atomic isotope of xenon with 129 nucleons \\
\hline${ }^{131} \mathrm{Xe}$ & The electric-quadrupole-sensitive isotope of xenon with 131 nucleons \\
\hline$A$ & The amplitude used in fitting signal profiles \\
\hline$B$ & Magnitude of magnetic field strength \\
\hline$c$ & Speed of light through the vacuum, approximately $300,000,000 \mathrm{~m} / \mathrm{s}$ \\
\hline$\delta \omega$ & The symmetric frequency offset of the electric-quadrupole-coupled atoms \\
\hline$E$ & Photon energy or atomic transition energy \\
\hline$e$ & Euler's number, approximately 2.71827 \\
\hline$F$ & Atomic ground-term hyperfine energy level \\
\hline$F^{\prime}$ & Atomic excited-term hyperfine energy level \\
\hline$f$ & Frequency associated with atomic precession or stimulating drive \\
\hline$\gamma$ & Gyro-magnetic ratio, in units of $(\mathrm{rad} / \mathrm{s}) /$ Tesla or $\mathrm{Hz} / \mathrm{Gauss}$ \\
\hline$h$ & Planck's constant, approximately $6.626068 \times 10^{-34} \mathrm{~m}^{2}-\mathrm{kg} / \mathrm{s}$ \\
\hline$\hbar$ & The reduced Planck's constant, a.k.a. unit of quantization, value $h /(2 \pi)$ \\
\hline$I$ & Maximum possible magnitude of isotopic nuclear angular momentum \\
\hline$J$ & Maximum possible magnitude of total electron angular momentum \\
\hline$\lambda$ & Wavelength of EM radiation \\
\hline$L$ & Maximum possible magnitude of electron orbital angular momentum \\
\hline$l$ & Orbital quantum number specifying electron orbital angular momentum \\
\hline$L N$ & The natural logarithm, outputs value one when evaluated at e \\
\hline$M_{F}$ & Fully defined atomic energy state for a specific hyperfine sublevel \\
\hline$v$ & Frequency of EM radiation \\
\hline$n$ & Principal quantum number specifying ground-term electron orbital \\
\hline$\omega$ & The frequency used in fitting signal profiles \\
\hline$\pi$ & The ratio of circumference to diameter of a circle, approximately 3.14159 \\
\hline$\pi^{0}$ & Linearly polarized light \\
\hline$P$ & First excited atomic energy term \\
\hline$\sigma^{+}$ & Right-hand circularly polarized light \\
\hline$\sigma^{-}$ & Left-hand circularly polarized light \\
\hline$S$ & The ground atomic energy term \\
\hline$s$ & Spin quantum number specifying electron spin angular momentum \\
\hline$\tau$ & Lifetime of interest in fit functions \\
\hline$t$ & Time after the assigned start of a test \\
\hline$T_{1 / 2}$ & Half-life, related to lifetime by a factor of the natural logarithm of two \\
\hline$T 1$ & Lifetime of the longitudinal spin component of z-polarized atoms \\
\hline$T 2$ & Lifetime of the transverse spin component of coherently precessing atoms \\
\hline$v$ & Vertical offset in fit solutions to account for noise in the data \\
\hline$V$ & Vertical offset in $T 1$ fit solutions, value typically A/2 \\
\hline$x, y, z$ & Right-hand orthogonal coordinate axes, pump laser on $z$ and sense on $y$ \\
\hline$y$ & Time-dependent signal amplitude \\
\hline$Z$ & Arbitrary atomic energy level \\
\hline
\end{tabular}




\section{SUBSRCIPTS AND SUPERSCRIPTS}

0

beat

C

drive

fit

$L$

Larmor

$m$

$n$

$Q$

$R$

Denotes the metric for $T 1$ derived from the apparent zero-crossing Related to the beating between two distinct frequencies in a signal Related to the center frequency for electric-quadrupole-coupled atoms Related to the stimulating drive Denotes the metric for T1 solved directly by the fit function Related to the low-frequency (left) peak in quadrupole-coupled data Related to the natural atomic Larmor precession Denotes the higher of two arbitrary atomic energy levels Denotes the lower of two arbitrary atomic energy levels Related to the quadrupole-induced frequencies Related to the high-frequency (right) peak in quadrupole-coupled data

\section{ACRONYMS}

$A C$

$D C$

$D P$

$E M$

FFT

$F P$

FID

GPS

$I M U$

LASER

$M E M S$

$M R I$

$N M R$

SNR

SWAP
Alternating Current

Direct Current

Delayed Pulse, industry standard method used to measure T1

Electromagnetic radiation, informally described as light

Fast Fourier Transform

Flipped Polarization, new method used to measure T1

Free Induction Decay, industry standard method used to measure T2

Global Positioning System

Inertial Measurement Unit

Light Amplification by Stimulated Emission of Radiation

Micro-Electromechanical Systems

Magnetic Resonance Imaging

Nuclear Magnetic Resonance

Signal to Noise Ratio

Size, Weight, and Power 


\section{Introduction to Navigation, Inertial Measurement, and Gyroscopes}

In our current age of advanced robotics, high-performance flight, and ambitious space exploration, the art of inertial navigation is a necessary focus for technological development. Even with the grand-scale navigation infrastructure established by the Global Positioning System (GPS), most military and science missions require motion and pointing control on a level of precision that can only be achieved with a dedicated onboard navigation device. Plus, many sensitive missions operate in areas where GPS is inaccessible, such as deep space, deep sea, underground, valleys and canyons, or places with exceptionally high electromagnetic interference, and for such cases, reliable independent navigation is crucial.

Modern automated navigation techniques rely heavily on inertial measurement units (IMUs), which employ translation-sensing accelerometers and rotation-sensing gyroscopes in three spatial axes to track the movement and heading of a vehicle as it propagates through space from some known starting location. IMUs allow an interplanetary rover to record its own motion, for science and for mission safety, far beyond the reach of GPS; they help the Hubble Space Telescope maintain focus on astronomically distant wonders while orbiting the Earth at nearly eight kilometers per second; they offer communications satellites the antenna pointing capabilities to deliver high-definition video to millions of televisions simultaneously with perfect clarity. There is no question of the utility of IMUs all around us in the world today. 
Especially for space applications, where every excess pound adds thousands of dollars to launch costs and extra watts of power add pounds in battery weight, some of the primary concerns among those who design and build IMUs are unit size, weight, and power consumption (SWAP). However, as with almost any technology, reducing these parameters tends to sacrifice performance, which is unacceptable in environments where GPS or other external positioning calibration tools are unavailable; low-performance IMUs accumulate significant errors quickly, and before long a vehicle may not function properly if its position and heading are not accurately determined. For most applications today this is of little concern because errors can be largely eliminated at regular intervals using GPS data for calibration, but we are focused on those special circumstances when that option does not exist. The quest to minimize IMU system resource requirements while preserving performance essentially relies on improving the individual sensors - the accelerometers and gyroscopes - that make up the total unit. Now, accelerometers are already well ahead of gyroscopes in the game of SWAP reduction, so newer, smaller approaches to gyroscope technology are in high demand.

One of the most popular modern approaches to building small gyros comes from the pursuit of micro-electromechanical systems (MEMS), which are now found in many smart phones and other entertainment-driven accessories. The main problem with MEMS gyros is that they rely on physical structures, like springs, which are inherently sensitive to noise from external vibrations. While they have demonstrated very high performance in the lab, MEMS gyros are not likely to be the best option for high-precision 
applications where significant vibration is typically an intrinsic part of the operating environment of the vehicle, as with most flight vehicles.

However, new approaches to atomic-based sensors may provide a fundamentally vibration-insensitive, MEMS-sized alternative that still meets navigation-grade gyro performance. Such endeavors are currently under research and development, but are quickly becoming more feasible and more promising; this discussion will focus on a particular type of atomic gyroscope which employs nuclear magnetic resonance (NMR) to track inertial rotations about a single axis, but first we should cover some of the history and general basics of gyroscope technology to fully appreciate the potential of the NMR gyro.

\section{A. History and Basic Operation of Gyroscopes}

The purpose of a gyroscope is to monitor angular deviations from some initial, fixed pointing orientation. Gyroscopes strapped down to a vehicle then provide information about the pointing orientation of the vehicle. Some gyros measure rotation angle directly, while others track rotation rates and calculate the angle from multiple rate measurements. In either approach, a gyro usually employs some physical structure that changes in a predictable way under the influence of an external rotation, such that when the gyro is turned about its sensitive axis, the angle through which it is turned can be determined based on the measured physical response to the rotation. For example, the first 
documented type of functioning gyroscope relied on the conservation of angular momentum of a spinning mass to make gyroscopic measurements.

There are actually two separate builds of this classic example: a rate gyro and a free gyro (which measures angles directly). A spinning mass gyro usually consists of an inner mass, which is driven by a motor to spin along a single axle, and an outer case which houses the spinning mass. With the rate gyro, there are actually three levels; the spinning mass is attached to an internal housing by a single axle and the internal housing is connected to the outer case also by a single axle orthogonal to the spinning mass axle, as shown in Figure 1 below (the drawings in this discussion are strictly intended for visualization of concepts; they are not drawn to scale, nor do they necessarily represent actual physical processes, unless otherwise noted).

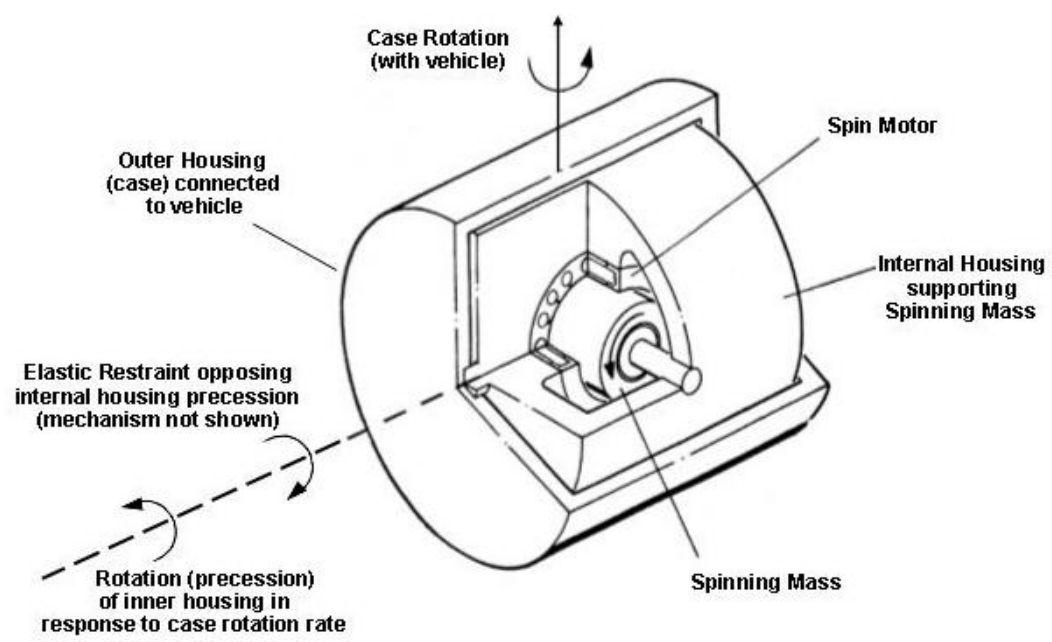

Figure 1. The spinning mass gyro as a rate gyro. When the spin axis of the inner mass is turned with the case, the inner housing rotates orthogonally to conserve angular momentum. An elastic restraint resists and measures the torque from the inner housing precession, thus yielding the case rotation rate about the sensitive axis. ${ }^{1}$ 
Turning the outer case about the sensitive axis (described as case rotation in Figure 1) induces precession of the inner housing about its axle and angular momentum is conserved. Usually, some restraint mechanism like a set of springs opposes the inner housing precession, providing a measurement of the induced torque and keeping the gyro in its operating range of orientations. Then, the angular rotation rate of the gyro about the sensitive axis is calculated from the measured torque.

The other type of spinning mass gyro is sometimes called a free gyro because the spinning mass makes no contact with the outer case; it is typically a sphere which is suspended from the housing and driven to spin either by high-pressure gas or electromagnetic fields. The idea here is that turning the outer case does not affect the spin axis of the inner mass because they are not attached and there is very little friction between them, so any rotation of the gyro adds a relative offset from the initial orientation between the two components. Pickoffs are used to measure the offset, yielding the gyro turn angle. The free gyro is shown in Figure 2 below. 


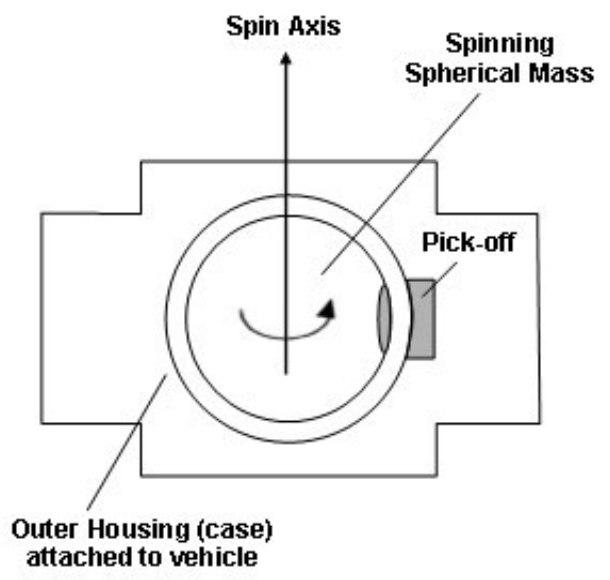

A

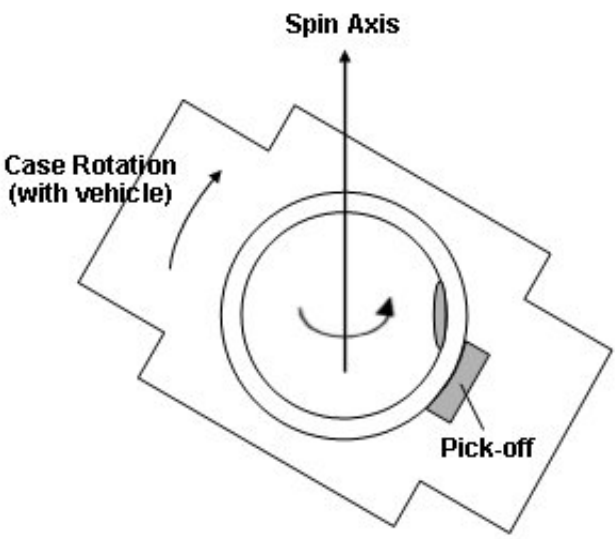

B

Figure 2. The free spinning mass gyro measures angle directly. The inner mass and outer housing do not make contact, such that rotating the unit preserves the spin axis of the inner mass and pick-offs detect the relative offset between the two layers of the gyro to give a measure of turn angle. ${ }^{1}$

The spinning mass gyro accomplishes the basic purpose of a gyroscope well, and both approaches offer some operational concepts analogous to the NMR gyro, as we will see later. However, these simple designs can be bulky and, more importantly, highly susceptible to noise from translational vibrations. Many other approaches to gyroscope technology have risen over the years, and the most successful ones have achieved impressive performance by rather innovative designs, but the basic goal to reduce SWAP while maintaining high precision always remains.

Even in its early phases of development, the NMR gyro seems to be a contender for performance and comes with considerable potential for SWAP reduction. Of course, to really compete with modern state-of-the-art gyros, the design and the research process behind it will still require improvements. To address the specific problem of this thesis 
demands a fairly detailed understanding of the principles of operation of the NMR gyro, so first an overview of the basic architecture and functionality will help focus our more rigorous discussion to follow.

\section{B. How the NMR Gyroscope Cell Test Apparatus Works}

The data presented in this paper was recorded from the NMR gyro cell test station, which is based on the architecture from phase two of the four-phase NMR gyro program. The cell test station is designed for convenient swapping and characterization of vapor cells as they get produced. Despite significant structural differences, the cell test station and the current phase-four gyro share the same operational principles. Keep in mind that references to specific hardware components or layout designs apply to the cell test station and may vary from the final NMR gyro assembly.

In very simple terms, the NMR gyro is similar to a free spinning mass gyro in that the signal is measured relative to the spin of a physical body. However, unlike the spinning mass gyro, in which there is truly a classical spinning mass, the NMR gyro relies on a group of polarized noble gas atoms contained in a small glass cell as the "spinning physical body". Of course, picking off a signal from something so subtle is no easy task; for now, suffice it to say that we can indeed monitor the net atomic spin of the polarized noble gas rather cleanly. 
Still, even with that assumption, how does the net spin of a group of atoms become a gyroscopic measurement? Well, recall the precession of the inner housing induced by the torque on the outer case of the spinning-mass rate gyro; a more familiar example of precession occurs with a spinning top on a desk. When the spin axis of the top points straight up, or more specifically, is aligned with Earth's gravitational field, the spin axis remains fixed and, by symmetry, gravity exerts no net torque on the top. However, as soon as the top hits a kink in the desk or gets perturbed in some other way that offsets its spin axis, the asymmetry relative to gravity results in a net torque, and just like in the rate gyro, the top begins to precess as shown in Figure 3 below, its spin axis rotating about the axis of the gravitational field in order to conserve angular momentum.

With that visualization now in mind, imagine we replace the spinning top with a group of atoms who all share a common spin axis and we change out gravity for a uniform magnetic field. The atomic population behaves in much the same way as the top; when the atomic spin points off from the direction of the field, the asymmetry in the atomic magnetic moment along the field axis results in a torque which causes the atoms to precess about the field, also illustrated in Figure 3. The stronger the magnetic field, the faster the atomic precession. 

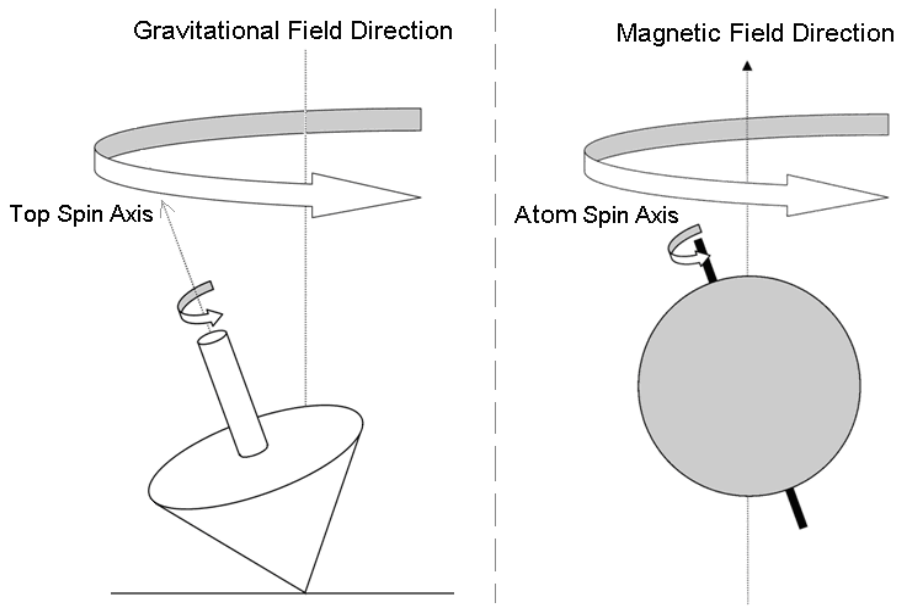

Figure 3. The spinning top (left) experiences precession about the direction of gravity in order to conserve angular momentum with the torque applied by gravity on the spin axis. A charged subatomic particle or atom with net spin (right) similarly precesses due to the torque applied on its magnetic moment by a magnetic field.

The atomic precession is the gyroscopic reference for the NMR gyro, but we must be able to maintain and measure the ensemble atomic spin in order to make use of it. Many intricate components are put in place to achieve this, but a simplified schematic including the main parts is shown below in Figure 4. Some terms in this brief synopsis may be unfamiliar and the reasoning behind the placement of components may be unclear; the main point for now is to highlight the key pieces that we are working with so that we can more easily reference and discuss them later.

The NMR gyro is, at its core, an atomic magnetometer which monitors the magnetic field changes generated by the net atomic spin precession. Measuring the spin of a single atom cleanly enough for a gyro signal is next to impossible, so we need to force many atoms into a common spin state in order to make a good measurement. For this we use a 
technique known as spin-exchange optical pumping, which aligns the spins of a relatively large portion of the atoms by absorption of very specific laser light.

Then, in order to make the atoms precess coherently, we need very stable and precisely controlled magnetic fields. We achieve this by enclosing the glass cell in a magnetic shield, which reduces external field influences and houses carefully designed coils capable of producing precise AC and DC magnetic fields on three axes around the cell. Current is driven through the coils using the outputs from external function generators. The coil set that generates the main DC field about which the atoms precess typically defines the $z$ axis, which should be very closely if not perfectly aligned with the pump laser. The $z$ axis is the sensitive axis for the NMR gyro, about which physical rotations of the device can be monitored. 


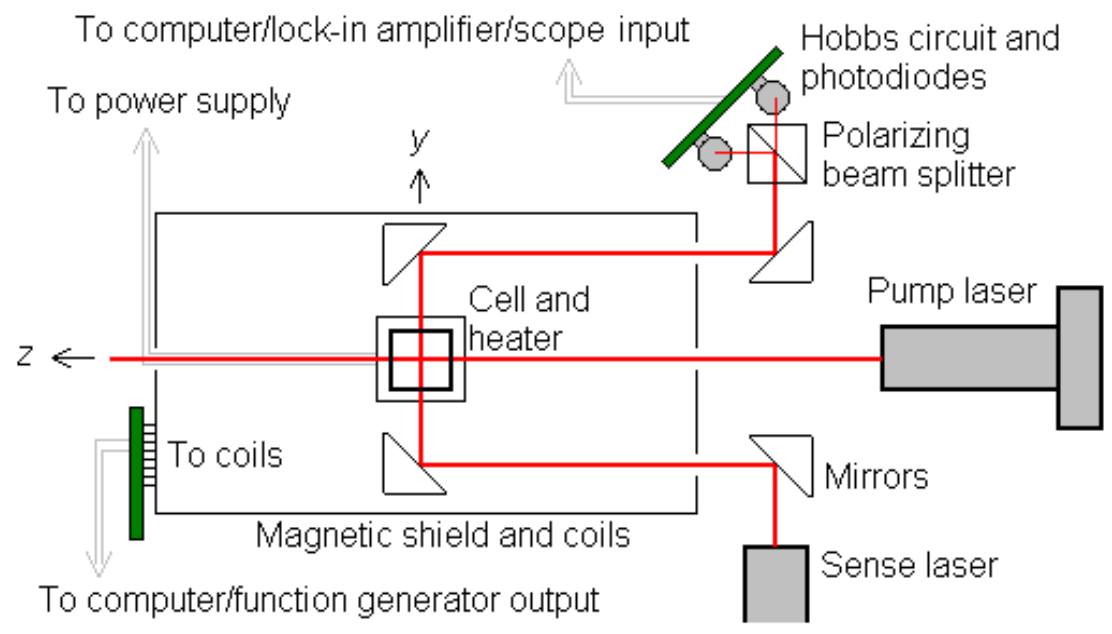

Figure 4. The major components of the cell test apparatus for the NMR gyro. The pump laser propagates along the $z$ axis, the sense laser along the $y$ axis. The shield reduces external interference and houses the coils that generate the magnetic fields necessary for NMR. The sense beam propagates along the $y$ axis through the cell, then gets split up to measure the magnetometer signal, which is then used to monitor the noble gas precession as a gyroscopic reference.

A second laser, the sense laser, propagates through the cell orthogonally to the pump laser, defining the $y$ axis. After passing through the cell, the sense laser is split into two beams of orthogonal polarizations using an optical device called a polarizing beam splitter, and the intensities of the two component beams are measured by photodiodes. The intensities are compared by a Hobbs circuit, which takes their sum and their difference by which we can determine the overall polarization axis of the sense beam after the cell. We will discuss this technique, called Faraday detection, in more detail further on. For now, accept that the sense polarization axis indicates the precession phase of the coherent alkali as stimulated by the noble gas atoms. The demodulated signal then directly represents the net transverse spin projection of the noble gas, which is compared to the reference signal generated by the gyro electronics. With the gyro in its original 
orientation, the two signals should match in frequency and phase; then, any phase offsets between the two signals indicate physical rotations of the gyro about $z$, analogous to the free spinning mass gyro. Also, as a rate gyro, any frequency offsets between the noble gas signal and the reference will indicate gyro rotation rates. Lastly, the temperature inside the cell affects gas pressures, which greatly influence signal strength and atomic spin lifetimes, so the cell sits atop a small heater capable of maintaining a fairly stable temperature.

This is the basic setup of the phase-two gyro and the cell test station on which this research was performed. We are now to the point where we leave cursory introductions and begin discussing things in proper detail. To do so, we must develop consistent terminology from the ground up, so some of what follows may seem redundant, but hopefully not much of it. 


\section{How the NMR Gyroscope Cell Test Apparatus Really Works}

In this section, the fundamental physical principles utilized in the NMR gyro design will be reviewed. The processes will be described as they apply to the gyro and should not be mistaken as uniformly applicable to all fields.

\section{A. Cornerstone Physical Principles in Achieving Magnetic Resonance}

An NMR gyro utilizes the spin behavior of a specific population of atoms under very carefully defined conditions to track single-axis rotations of a platform. Magnetic resonance is described by quantum mechanics, so a review of some key physical phenomena is in order. As often as possible, we will explore concepts from a classical perspective to avoid some of the unfamiliar subtleties of quantum mechanics. This approach may imply some erroneous technical details but is necessary to expedite our understanding of the core factors at work in the gyro.

\section{Particle Spin and Larmor Precession}

Particles, be they atomic nuclei, electrons, or any other subatomic species we now believe to exist, can display a seemingly inherent energy property analogous to the angular momentum of a classical spinning mass. Naturally, we call this particle property spin. 
Associated with a charged particle's spin is a magnetic dipole moment; thus, the spin generates a magnetic field. We can measure a particle's spin along a single axis, which we define as the quantization axis; we call it this because there are finite and quantized possible outcomes of such a measurement, each with a distinct probability in a given environment. Unlike a macroscopic spinning mass, which we presume to have a continuum of infinitely many possible spin states, a measurement of a particle's spin must return one of a very limited set of values. In theory, this makes the particle spin far more stable than the classical spinning mass; any perturbation in any direction can presumably alter the spin state of the classical mass, whereas only disturbances of specific direction and magnitude can influence the spin state of the particle. Of course, with approximately $10^{10}$ atoms moving around an 8-cubic-mm volume at high speeds, these specific disturbances still happen quite frequently in the form of atomic collisions. In a sufficiently well controlled environment, though, the net spin of an atomic group can be made quite stable.

Different particles have different possible spin states. Certain particle species have zero intrinsic spin; for others, zero spin is only one of multiple possible states. The electron by nature is a spin-1/2 particle, so the angular momentum due to its spin is either $+1 / 2$ or $-1 / 2$ along the quantization axis. As a note, particle energy values are almost always given in units of the reduced Planck's constant, denoted $\hbar$, also known as the unit of quantization. An electron of spin angular momentum $+1 / 2$ is often referred to as spin-up, because its spin vector contains a component in the positive direction of the quantization axis. By contrast, one of spin angular momentum $-1 / 2$ is called spin-down. Meanwhile, atomic 
nuclei can have much higher-order spins. For example, the nucleus of a cesium atom is a spin-7/2 particle, meaning its quantized spin angular momentum can take on any value in the set $[-7 / 2,-5 / 2,-3 / 2, \ldots,+7 / 2]$.

Closely tied in with spin is Larmor precession, the foundational principle in magnetic resonance. A particle placed in a magnetic field that points offset from the particle's quantized spin axis experiences precession about the axis of the field, as illustrated in Figure 1 below.

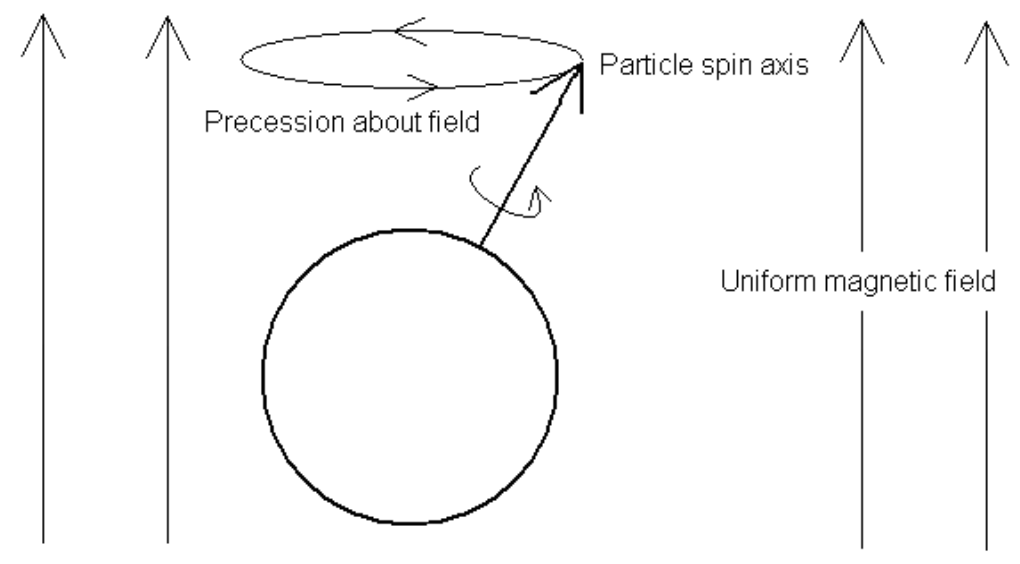

Figure 5. Larmor precession of a particle about a magnetic field offset from the particle's spin axis.

If the magnetic field is steady and uniform then the atomic precession frequency is constant; we call it the Larmor frequency, described by

$$
f_{\text {Larmor }}=\gamma B /(2 \pi),
$$


in $\mathrm{Hz}$, where $B$ is the magnetic field strength and $\gamma$ is the gyro-magnetic ratio which is unique for every single known atomic isotope. Also, the precession direction relative to the magnetic field vector varies between isotopes (even between the two isotopes of xenon used in the NMR gyro cells), which is reflected in the vector formulation of equation $i$ by the value of $\gamma$ as positive or negative. For a more formal description of Larmor precession, see references. ${ }^{2,8}$

Therefore, by monitoring a gas sample in a magnetic field of known strength, the elements that compose the gas can be identified by their precession frequencies. This is the basis of NMR spectroscopy and some magnetic resonance imaging (MRI) techniques. Or, with a gas of known composition, the precession frequencies can be measured to determine the strength of the magnetic field: an atomic magnetometer.

However, although the frequency is constant, we have not yet discussed anything to govern the phase of the atomic precession, and without coherent phase we cannot monitor the overall behavior of the gas sample. See, in a normal distribution, any potential signal generated by the precession of one atom would be negated by signal from another atom in the opposite phase of precession, and thus the total gas sample would produce no measurable signal if there is no coherent phase in the atomic group. In fact, as far as we have discussed, there would be no reason even for preferential atomic polarization; roughly half of the atoms would be spin-up and the other half spin-down at any time. Figure 6 illustrates the difference between what we start with and what we want. 

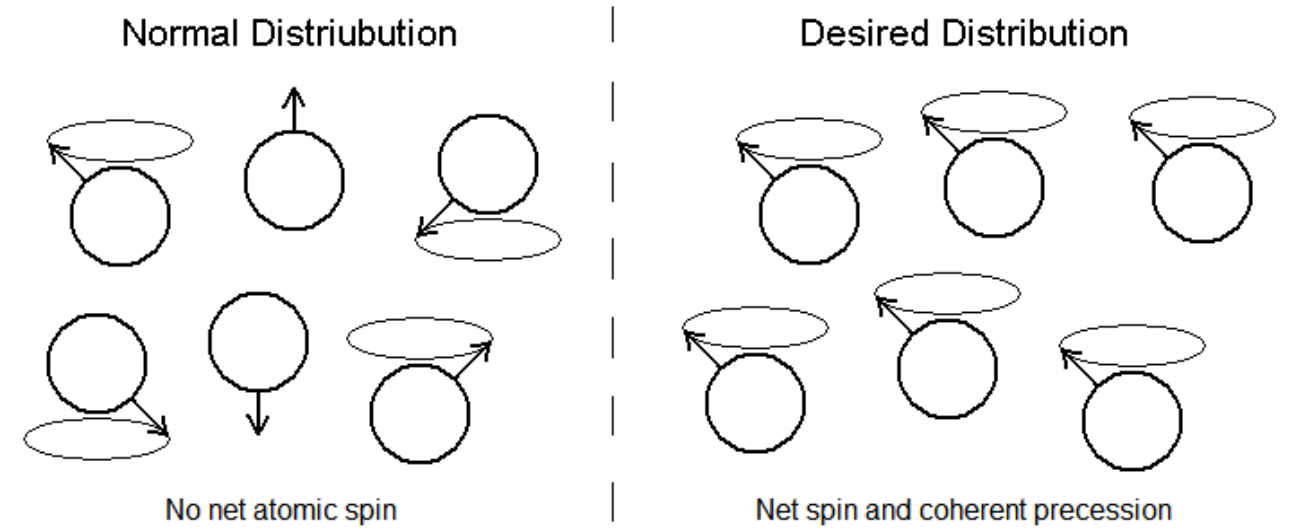

Figure 6. In a typical equilibrium distribution, the noble gas atoms do not share a common bias in spin orientation. Although the atoms may individually precess, without a net common spin axis we cannot measure anything.

The first step, then, is to force a large portion of the gas population into a particular spin orientation. For this, we turn to a technique called spin-exchange optical pumping of atoms, an increasingly important topic of study and an extremely useful approach to preparing NMR samples. However, a few more key principles must be understood to fully appreciate the process of optical pumping.

\section{Relevant Properties of Electromagnetic Radiation}

Early in the $20^{\text {th }}$ century, the traditional perception of light and matter as continuous entities was being cast off as scientists began exploring quantum physics to describe the intimate relationship between the two substances. Although the existence of atoms was already highly regarded as fact, the question of exactly how atoms interact with one another, and with electromagnetic (EM) radiation, was in heated debate. To explain how 
energy was transferred to an atom in an EM field, some physicists treated the field as a compilation of discrete bundles of energy called "wave packets" which we now commonly refer to as photons or particles of light, where energy transfer was assumed to occur by collisions between atoms and these photons. There are certain scenarios where it is only possible to accurately describe light in terms of photons, and others where light must be considered a field; to this day, the mystery of wave-particle duality remains one of the largest ambiguities in our understanding of our universe.

Regardless, there are several important aspects of light that characterize the nature of photon-atom interactions. First we have the frequency of the EM wave, which defines the average energy that the photons carry by the relationship

$$
E=h v=h c / \lambda
$$

where $h$ is Planck's constant and $v$ is the frequency of the EM radiation; in the second description, $v$ is replaced by its representation as the speed of light through vacuum, $c$, divided by the wavelength of the EM energy, $\lambda$. In vacuum, frequency and wavelength can both be used to describe photon energy, but through any other medium, the wavelength and speed of light change, so the frequency is the only single parameter that always truly describes the EM energy.

The second property that characterizes radiation is polarization, which describes the relative phase between the electric and magnetic fields that compose the light. Just like 
particles of matter, photons can carry angular momentum along a specific axis. When the two fields match in phase or are 180 degrees apart, we say the light is linearly polarized, denoted by $\pi^{0}$, which means it carries no angular momentum in the direction of motion of the photons (the propagation direction). When the two fields are 90 degrees apart in phase, the light is circularly polarized and the angular momentum vector either points along the direction of propagation, which we'll call right-hand circular and denote $\sigma^{+}$, or opposite it, which we'll call left-hand circular and denote $\sigma^{-}$. Figure 7 helps illustrate these polarization orientations.

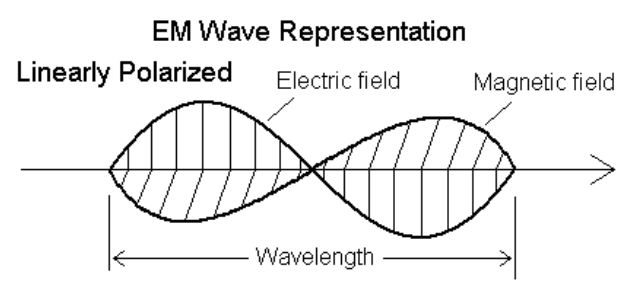

Photon Representation

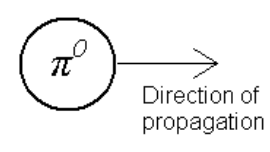

Right-Hand Circularly Polarized
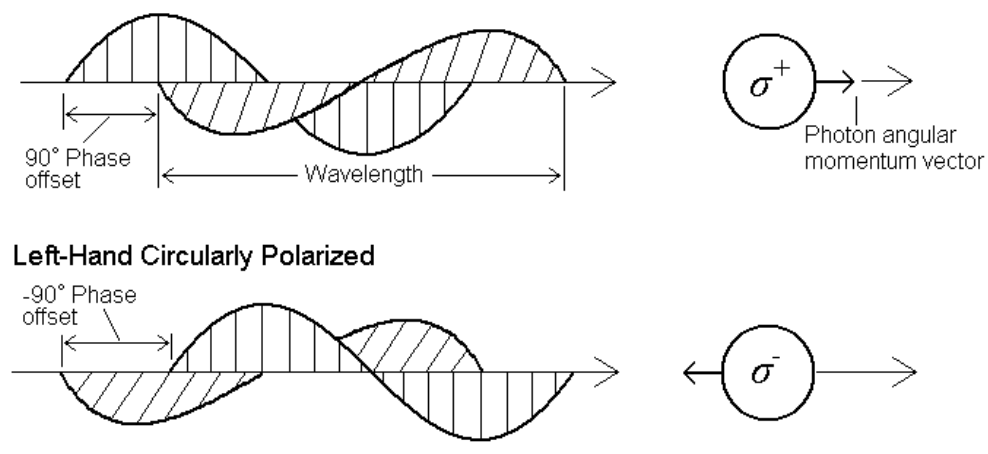

Figure 7. Relevant light polarizations. When the phase of the electric and magnetic fields coincide, the light is linearly polarized and the photon has no angular momentum in the direction of propagation of the light. When there is a relative phase offset of 90 degrees, the light is circularly polarized and the angular momentum vector either points along the direction of propagation if righthand circular or opposite it if left-hand circular. Anything in between has elliptical polarization. 
If the relative phase of the fields is something between zero and 90 degrees (or between 90 and 180 degrees), the polarization is elliptical and can be represented as a combination of circular and linear light. In fact, there are two mathematical bases by which light is typically described. In the linear-orthogonal basis, the polarization is described by its components along two orthogonal axes (usually called horizontal and vertical); in the circular basis it is described by a combination of $\sigma^{+}$and $\sigma^{-}$, where $\pi^{0}$ light is considered equal parts $\sigma^{+}$and $\sigma$ and the polarization axis depends on the relative phase between the two parts as in Figure 8 below. ${ }^{3}$

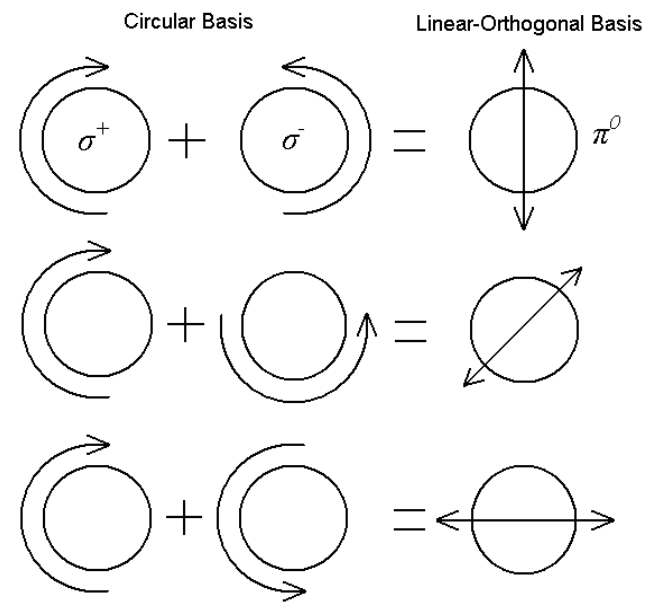

Figure 8. Linearly polarized light is represented as equal parts left and right circular light, where the relative phase between the two circular components determines the polarization axis. The direction of propagation of light is assumed to be into the page.

Now, there are some useful tools to alter or utilize specific polarizations of light. For example, a quarter-wave $(\lambda / 4)$ plate is made from a sheet of birefringent material, which maintains a higher index of refraction in one axis than in the other, such that in the correct orientation the electric and magnetic fields travel at different speeds through the 
plate and the relative phase between the fields is therefore altered. A $\lambda / 4$ plate of the correct thickness, angled appropriately relative to the polarization axis of incident light, can induce a 90-degree phase offset and effectively convert linear light to circular or vice-versa. If the plate is made twice as thick, it makes a half-wave $(\lambda / 2)$ plate, which maintains linear polarization but rotates the polarization axis. Another useful tool is a polarizing beam splitter. If we allow the orientation of the beam splitter to define the linear-orthogonal axes, it transmits $\pi^{0}$ light that is vertically polarized and reflects $\pi^{0}$ light that is horizontally polarized. If the light is circularly polarized or the linear polarization axis of the light is 45 degrees (equal parts vertical and horizontal), the light will be split, half transmitted and half reflected. Figure 9 illustrates the functionality of these optical devices.

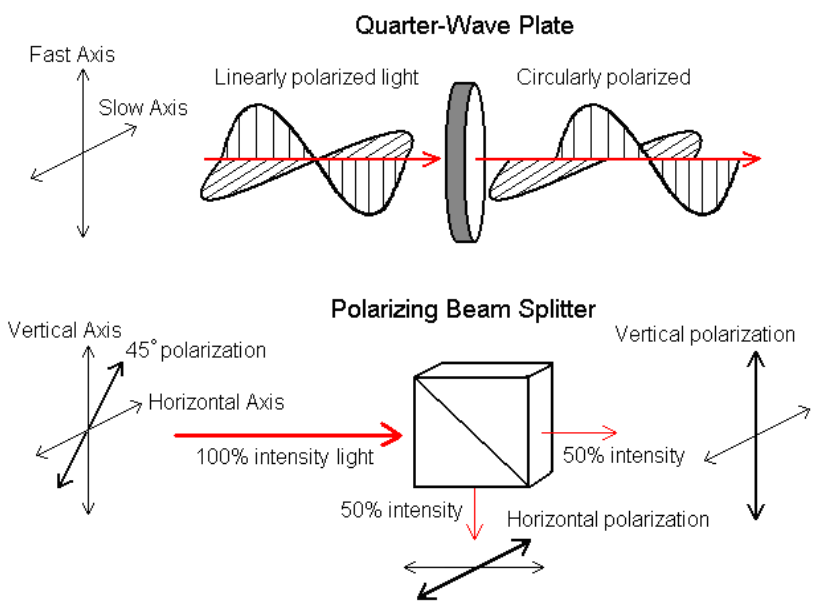

Figure 9. A quarter-wave plate (top) converts the polarization of light between linear and circular for a certain range of wavelengths of incident light when the fast and slow axes of the birefringent plate are aligned appropriately with the EM waves. A polarizing beam splitter (bottom) splits incident light into separate components of vertically and horizontally linearly polarized light. 
Now that we have covered the relevant properties of EM radiation, we can discuss the relevant details of interaction between light and matter.

\section{Quantized Atomic Energy Transitions and Optical Pumping}

In 1917, Albert Einstein defined absorption of radiation as the process by which an atom at energy level $Z_{n}$ with corresponding energy $E_{n}$ jumps to a higher energy level $Z_{m}$ with

energy $E_{m}$ by absorbing a photon of energy $\left(E_{m}-E_{n}\right)$. An atom drops back down to level $Z_{n}$ by emission of a photon of the same energy. ${ }^{4}$ Radiative emission is either spontaneous or stimulated by another incoming photon, and in fact Einstein's predictions led to the invention of Light Amplification by Stimulated Emission of Radiation (LASER). The technique of optical pumping depends on the atomic absorption of laser light to influence the energy states of atoms, and if setup correctly it can influence the angular momentum (spin) states of the atoms as well.

The state of an atom is defined by several levels of possible energy, given some potential field in which the atom resides. Now, consider a single atom with a sole valence electron; all the orbital levels except the outermost have every possible electron spin orientation occupied. In other words, the only variable parameters are the valence electron orbital level and the spin orientation between that electron and the nucleus (and the total atomic spin relative to the magnetic field). The alkali metals all have this electron structure, 
which allows us to use the relatively simple hydrogen model to predict the magnitudes of separation of the possible energy levels of the alkali atoms.

For example, the atomic configuration of cesium is $[\mathrm{Xe}] 6 s 1$; it has an ion core with the electron structure of a noble gas xenon atom (which has absolutely every electron slot filled), plus one additional electron outside that core in the $6 s$ orbital level. Likewise, rubidium has the configuration of a Krypton atom plus one valence electron in the $5 \mathrm{~s}$ level. (Some of the results to be presented in this discussion were gathered using cesium as the alkali metal, while others were collected using rubidium; there should be no difference between the two alkali species for our purposes.) With either alkali, the valence electron spends nearly all of its time outside the ion shell, composed of the nucleus and the inner electrons, so the atom can be treated as a two-body system. Figure 10 illustrates this visualization. The two-body approximation allows for very accurate predictions of the atomic transition energies based on hydrogen-like approximations, which facilitates the experimental setup when procuring lasers of the correct frequency range and coils to produce the appropriate magnetic fields.

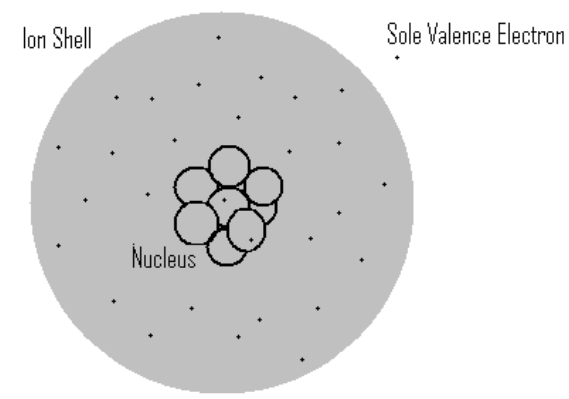

Figure 10. A single valence electron remains outside the ion core of the alkali atom, analogous to the two-body hydrogen model 
The electron state carries enormous influence on the atomic energy level. In general, the energy level is classified first by the electron orbital levels, which compose the gross structure of the atom. An atom at the lowest orbital level is said to be in the ground term; an electron at a higher orbital level is in an excited term.

However, finer inspection shows that the orbital levels are split into smaller sublevels due to coupling between the magnetic field from the orbit of the charged electron about the nucleus (or vice versa, depending on the frame of reference) and the magnetic moment from the spin of the electron itself; we call this spin-orbit coupling. These sublevels, which make up the fine structure of the atom, are separated by energy differences that are orders of magnitude smaller than the energy differences that define the gross structure.

Even further splitting in the fine-structure sublevels arises from coupling between the magnetic moments from the spins of the nucleus and the electron, called spin-spin coupling. These levels, smaller yet in separation, make up the hyperfine structure.

Subjecting the atom to an external magnetic field results in even finer splitting, the Zeeman Shift, due to the relative orientation between the total magnetic moment of the atom and the applied field (the atom must have a higher energy if its magnetic moment opposes the external magnetic field). A similar effect is observed under an external electric field, which is referred to as the Stark Shift. 
Until the atomic energy level is specified down to the Zeeman (or Stark) sublevel, the full state of the atom is not defined. Figure 11 depicts the splitting of the lowest energy levels of the natural-abundance cesium atom, an alkali metal commonly used in atomic applications.

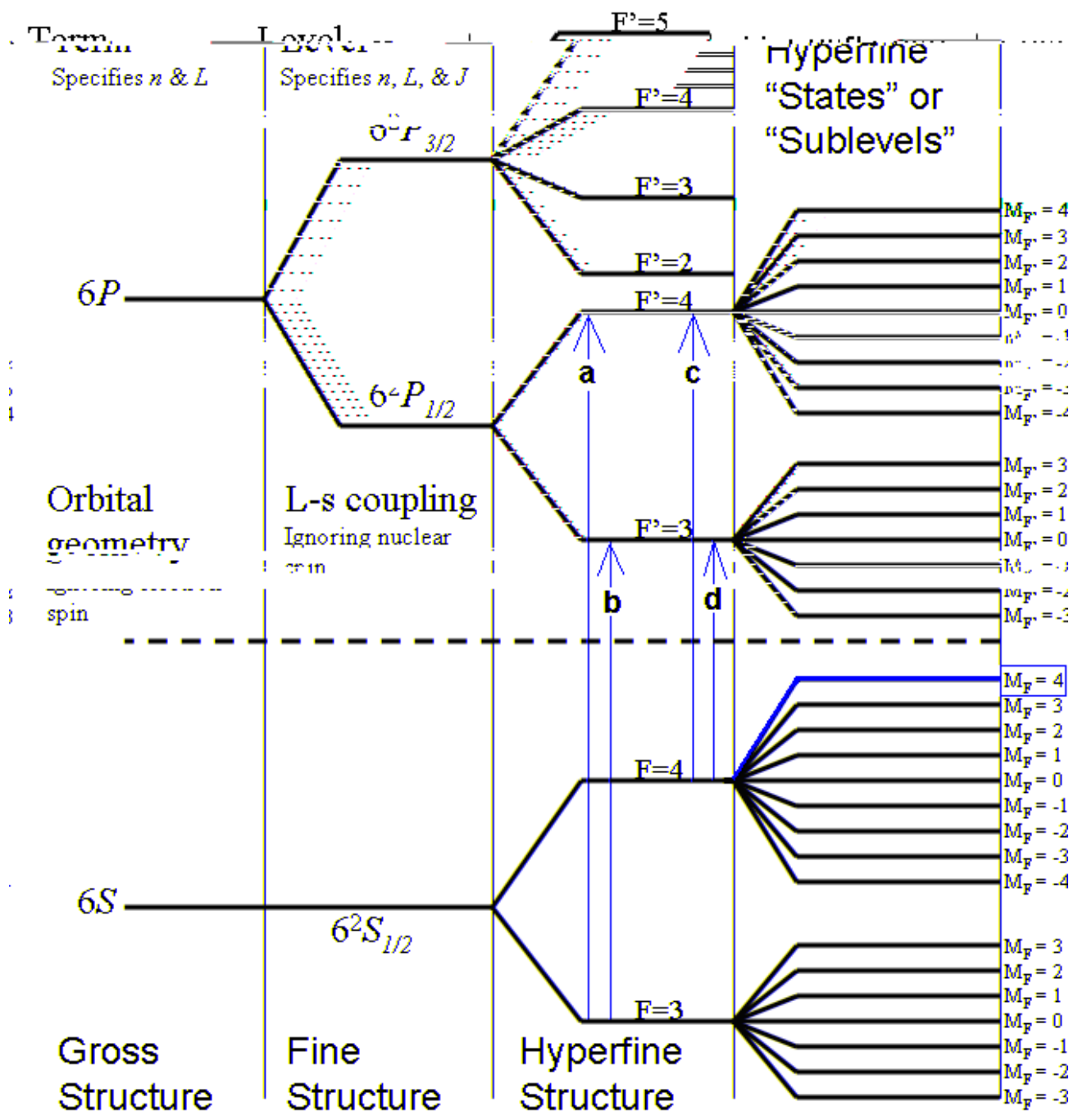

Figure 11. Basic layout of the energy level structure of a cesium atom. The red arrows indicate the transitions of interest for the NMR gyro. Figure 12 shows physical evidence of these transitions. The highlighted $M_{F}=4$ state in the $F=4$ hyperfine level is the destination state in our implementation of optical pumping. 
To understand the important role that these possible energy levels play in optical pumping, we must first clearly define what it means for an atom to be in a specific state. The ground-level $6 S$ electron has no orbital angular momentum, so the total electron angular momentum in that level depends only on spin. Therefore, the $F=3$ and $F=4$ hyperfine sublevels in the $6^{2} S_{1 / 2}$ fine level represent the spin-down and spin-up electron states, respectively, relative to the nuclear spin. We have the same scenario in the lowest fine level of the first excited gross term, the $6^{2} P_{1 / 2}$ fine level, denoted as the $F^{\prime}=3$ and $F^{\prime}$, $=4$ hyperfine sublevels. On the other hand, in the higher fine level of the first excited term, the $6^{2} P_{3 / 2}$ fine level, the total electron angular momentum is defined by both the orbital angular momentum and the electron spin, which allows for more possible atomic spin states; the total spin-orbit angular momentum of the electron in this fine level can be any value in the set $J=[-3 / 2,-1 / 2,1 / 2,3 / 2]$, and the corresponding total atomic angular momentum values, including the nuclear spin, are represented as $F^{\prime}=2,3,4$, and 5 in the $6^{2} P_{3 / 2}$ fine level. Each of these hyperfine levels, in the presence of an external magnetic field, experiences Zeeman splitting, separating into integer-incremental levels of $-F \leq M_{F}$ $\leq F$, dependent upon the orientation and magnitude of the atom's total angular momentum vector relative to the magnetic field. The fully defined spin state of the atom is represented by the $M_{F}$ of a specific hyperfine level in a given field, since the net field defines the quantization axis and the magnitude of the Zeeman splitting.

Optical pumping gives us the ability to induce atomic state changes by absorption of laser light. We can use light of the proper frequency to excite atoms as described by Einstein, so with a group of atoms like our alkali sample we can alter the populations of certain 
energy states. In general, given a large enough group of atoms, $F=3$ and $F=4$ will be equally populated under standard equilibrium. However, by optically pumping atoms out of one ground sublevel (say, $F=3$ ) into an excited level, the total ground population can be made to favor the other ground sublevel $(F=4)$, essentially biasing the ground-level equilibrium.

To accomplish optical pumping, the energy of the photons from the laser must be at or very close to the energy of a true and allowed atomic transition. The theoretical energy difference between two specific atomic energy levels can be approximated by solving Schroedinger's equation for the two levels with the assumption that the alkali behaves like a two-body system. For the purpose of focusing this paper, the math is unnecessary, but it is noteworthy that theoretical predictions and experimental results are extremely consistent in matters such as these.

In our implementation, a laser source is tuned to the correct frequency (or wavelength) to excite cesium atoms out of $F=3$ to the $6^{2} P_{1 / 2}$ fine level, either to $F^{\prime}=3$ or $F^{\prime}=4$. The excited atoms will, typically through spontaneous emission, decay back down to either the $F=3$ or $F=4$ ground sublevel with a distinct probability for each. As the $F=3$ atoms continue to be optically pumped while $F=4$ is left nominally undisturbed, the population distribution gets shifted to a new equilibrium that favors $F=4$ as the dominant ground hyperfine level. 
Now, there are two distinct wavelengths of light that will pump atoms from $F=3$ to the first excited level; one wavelength pumps up to $F^{\prime}=3$ and one pumps up to $F^{\prime}=4$. Likewise, there are two distinct wavelengths to excite atoms from $F=4$ as well, as indicated by the red arrows in Figure 11. We can confirm these transitions experimentally by shining a laser through a glass cell filled only with alkali vapor. We record the intensity of light that passes through the cell as we increase the output wavelength of the laser, sweeping through the relevant alkali transition wavelengths, to obtain the transmission profile. Figure 12 below shows the results for such a test across a spectrum of laser wavelengths through a cesium vapor reference cell in comparison with a typical NMR gyro test cell, which also contains xenon and other gases mixed in with the cesium. Since there is already a photo-detector in place for this, we use our sense laser in our apparatus, which propagates along the $y$ axis. When the laser light is far off from one of the transitions, nearly all of the light is transmitted straight through the cell, giving us close to $100 \%$ intensity at the photo-detector. The dips in intensity, labeled $a$ through $d$, correspond to the labeled transitions in Figure 11; the cesium atoms absorb the laser light near those wavelengths, thus making the corresponding energy transitions, and the light that they absorb never reaches the photo-detector. 


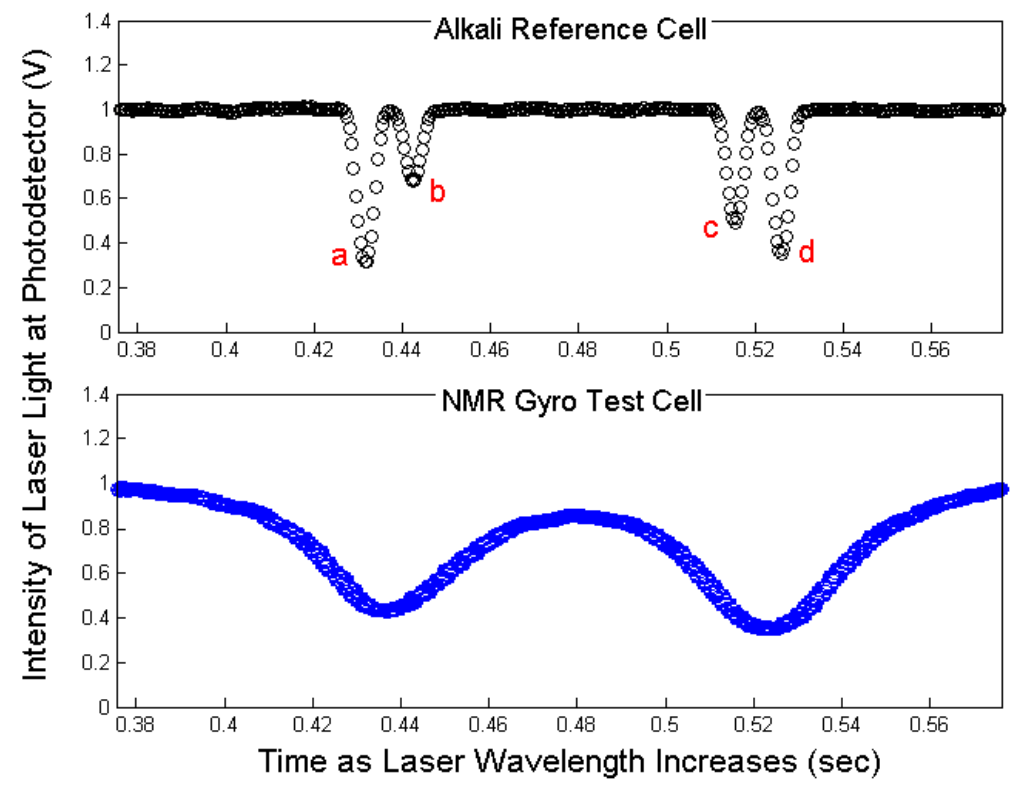

Figure 12. Transmission intensity profiles of laser light after passing through two test cells. The top chart shows the profile for a cell with cesium vapor only; the bottom shows a gyro test cell with a cesium, xenon, and buffer gas mix. The laser is swept through the resonance wavelengths, indicated by the dips in intensity which are labeled corresponding to the transitions illustrated in Figure 11. Note the effects of pressure broadening, quite apparent in the gyro test cell.

The thin natural line widths of the alkali reference absorption dips would be even thinner if not for Doppler broadening; at the time of absorption some of the atoms are moving toward the oncoming photons, so the momentum of the photons relative to these specific atoms is higher than that relative to the average of the entire atomic group. The increased relative energy is seen as an upward shift in the frequency of the laser light as described by equation $i i$, so light of a slightly lower frequency relative to the average of the entire atomic group is more likely to excite the transition for atoms moving toward the light. By contrast, for atoms moving away from the oncoming photons, the relative energy decrease causes light of slightly higher frequency to excite the transition. Therefore, the atomic vapor as a whole absorbs light not only at the transition wavelengths but also 
slightly above and below them, thus broadening the absorption lines. Furthermore, including atoms of other gases in the cell, as in the NMR gyro test cell, increases collision rates and thus widens the lines so much that the four dips blur into two (or sometimes just one, with sufficiently high buffer gas pressure). We call this pressure or collision broadening, and it can actually be a powerful tool for measuring the buffer gas pressure inside the sealed test cells.

As part of my research, I established a consistent means for extracting this information, which has been very important for making informed decisions on how to construct higher performance NMR gyro cells. The method is as follows.

We construct absorption profiles for both cells by applying the negative of the natural logarithm to the transmission intensity profiles in Figure 12. We then find the Dopplerbroadened width of the alkali absorption lines by fitting the reference cell absorption profile with the sum of four Gaussian curves, as displayed in green in the top chart of Figure 13 below. The solver takes the theoretical transition line centers and amplitudes as inputs to find the proper Gaussian width. 


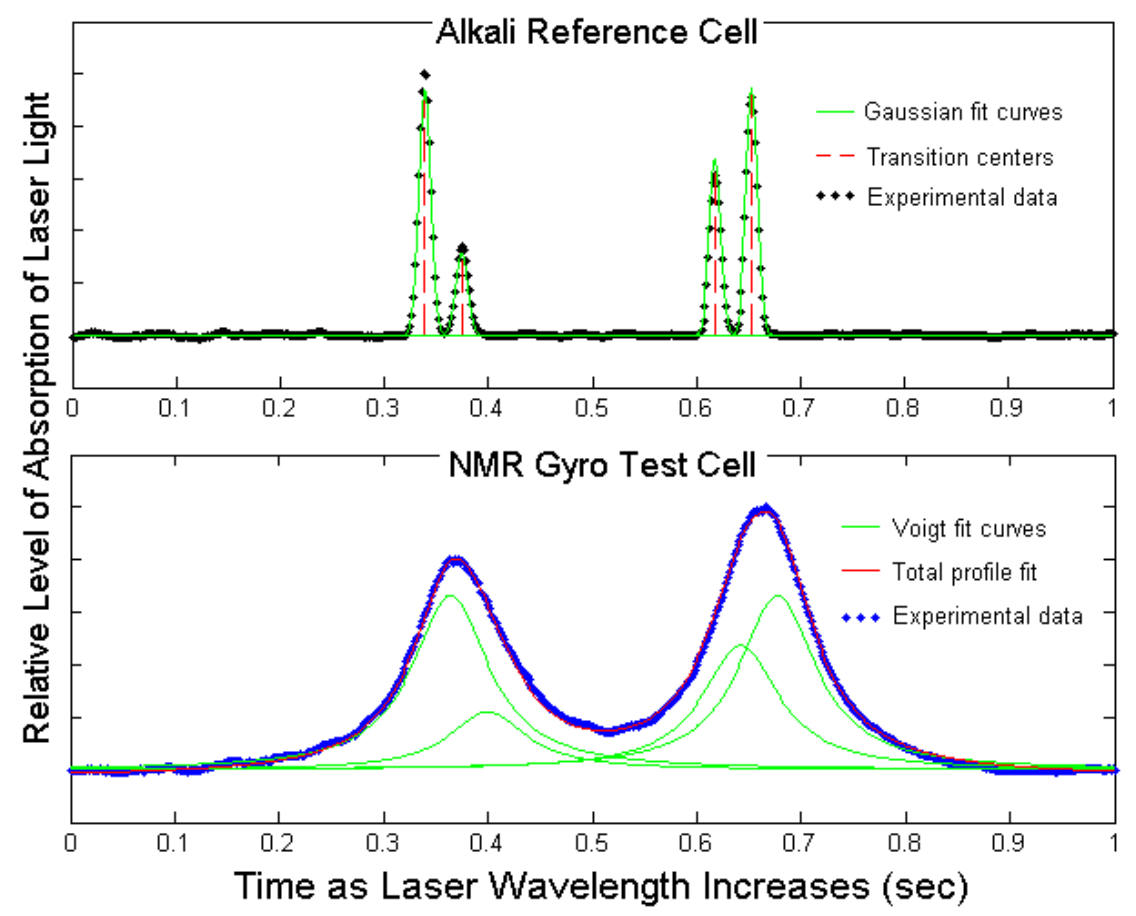

Figure 13. The absorption profiles constructed from the transmission intensity profiles in Figure 10 are fitted using Gaussian curves (top) and Voigt profiles (bottom) to determine gas pressures in gyro cells. The time values in the data have been normalized.

Collision broadening, on the other hand, is a Lorentzian effect. In order to fit the absorption profile for the gyro cell, we use an approximated convolution of Gaussian and Lorentzian curves called a Voigt profile, shown green in the bottom chart of Figure 13. To construct appropriate Voigt profiles, we use a free MATLAB function available on MathWorks database to approximate the faddeeva function (also known as the plasma dispersion function), which is essentially a Voigt profile without scaling. ${ }^{5}$

We use the Gaussian width solution from the reference cell profile and find the correct Lorentzian width such that the sum of the four Voigt curves (the total fit shown in red in the bottom chart) matches the actual NMR cell absorption profile. Once a match is found, 
the associated Lorentzian width value linearly corresponds to a distinct buffer gas pressure inside the cell. In fact, we were able to measure our own in-lab pressure-towidth correlation while filling a test cell, which matched closely with accepted theoretical values.

This technique has proven quite useful in studying and improving the gyro cell filling and sealing process because when sealing a cell (using a torch), gases get moved around and the final sealed pressure is often different from what was intended. Design changes can only be confidently applied if observed changes in cell performance can be linked back to measured cell pressures.

Returning to our discussion of optical pumping, we can now incorporate a second laser along the direction of the magnetic field- our pump laser - and tune it to pump atoms out of $F=3$ (the dip composed of absorption lines $a$ and $b$ ). If we repeat the absorption test from Figure 12 for the gyro cell while pumping $F=3$, we can clearly see evidence of the shift in energy level populations in Figure 14 below, where the $F=4$ dip is much deeper when the pump laser is actively pushing atoms out of $F=3$ and into $F=4$ during the sense beam sweep. 


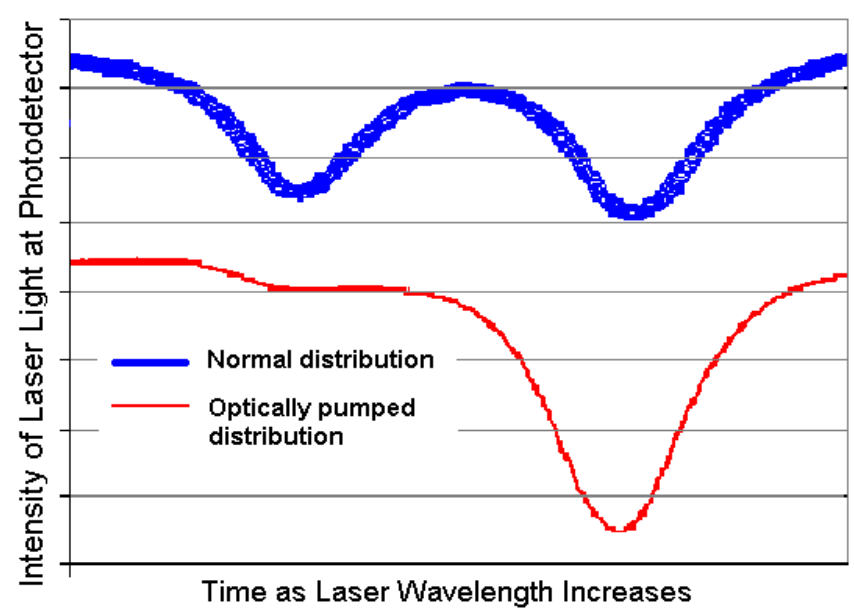

Figure 14. Repeated experiment from Figure 12 with $F=3$ being pumped for the gyro test cell. The $F$ = 4 population dominates the ground term as the pump laser drives the process moving atoms out of $F=3$ and into $F=4$. Time and intensity values were not scaled between the two profiles, so they were removed for irrelevance; the apparent vertical offset is just for ease of viewing. Only the relative shape of the two profiles is important here.

Pumping $F=3$ increases the population of the $F=4$ level and thus increases the level of absorption of the sense laser light in that range of wavelengths. When the pumping reaches equilibrium, some percentage of the alkali atoms in the cell are excited, but an atom only remains in an excited state for a brief time before decaying back down to ground through emission. For pure spontaneous emission, two thirds of the excited atoms decay and return to $F=3$, while the other third decay to $F=4$, so the pumped equilibrium has far less $F=3$ atoms and also more $F=4$ atoms than the no-pump scenario, as proven by the respective transmission intensity dips. 
But this all involves only the hyperfine levels and has nothing to do with atomic spin orientation relative to the magnetic field, which sparked our discussion of optical pumping in the first place.

\section{a. Spin-Exchange Optical Pumping}

Now, if the pump laser light is circularly polarized then the photons carry angular momentum along the pump axis. When an electron absorbs such a photon, the angular momentum of the photon is transferred to the electron and total angular momentum is thus conserved. Formally, this means that upon absorption the atom jumps to a different Zeeman level in the excited term than it occupied at ground. During the time that the electron is excited, it has a chance of interacting with the nucleus of the atom, in turn transferring its spin angular momentum to the nucleus; the nucleus acts as a reservoir of angular momentum as the pumping continues adding more and more angular momentum along the pump axis to the alkali gas population. Eventually, typically within microseconds, a steady state is reached in which a certain population of the alkali atoms is at maximum polarization.

Since the pump laser emits $\sigma^{+}$light and points in the direction of the main magnetic field, the fully polarized atoms occupy the $F=4, m_{F}=4$ state (recall Figure 11); if the pump light polarization is reversed $\left(\sigma^{-}\right)$or the propagation direction of the laser and the magnetic field are anti-aligned (both of which are indeed the case during some of this 
research) then the fully polarized atoms occupy $F=4, m_{F}=-4$. In either case, the fully polarized atoms can no longer absorb any photons from the pump laser because the $6^{2} P_{1 / 2}$ fine level has no higher Zeeman level to which they can transition; in other words, absorption cannot occur because it would violate conservation of angular momentum.

Once in this polarized state, atoms are eventually depolarized by collisions, either with the cell walls or with other gas atoms in the cell, which are frequent and often result in completely random alkali spin states. We can convince ourselves, given a laser of finite power, that there is a limit to the amount of pumping possible; that is, the overall portion of the alkali that can be simultaneously spin-polarized is limited. With our experimental conditions, roughly $5 \%$ to $20 \%$ of the vaporized cesium atoms in the cell occupy the $F=$ 4, $m_{F}=4$ (or $m_{F}=-4$ ) state at the pumped equilibrium. This polarization level is low compared to pure cesium cells, which for the same pump laser intensity can reach above $90 \%$ polarization, because our cells are designed for polarized alkali atoms to interact with other atoms and thusly transfer their polarizations through spin-exchange. ${ }^{5}$ In particular, we use two isotopes of xenon gas, ${ }^{129} \mathrm{Xe}$ and ${ }^{131} \mathrm{Xe}$, for spin-exchange with the alkali. Some of the spin angular momentum from the pumped alkali population gets transferred to the xenon atoms through collisions and thus induces a net spin bias on the xenon population in the cell. We also include nitrogen gas in the cell, but that is a buffer gas and not a part of the nuclear magnetic resonance measurements; its primary purpose in the optical pumping process is to increase the relative probability of alkali spin exchange with xenon by the three-body-process formation of short-lived Van der Waals molecules, which significantly enhance the spin-exchange rate through increased 
interaction time between the alkali and the noble gas. The ratio of nitrogen to xenon acts as a control on the overall spin-exchange rate which greatly influences the longitudinal spin relaxation time of the xenon group - a matter we will later get into deeply. So, even though the laser only interacts with the alkali atoms, in the long run we are able to spin polarize other species as well, albeit to a much lesser extent. We estimate that the level of xenon polarization is about $5 \%$ to $20 \%$ that of the alkali, so only $0.25 \%$ to $4 \%$ of the xenon population in the cell has coherent spin at any time. Still, the field effects necessary for gyro operation are easily measured from that small group.

So to summarize our implementation of optical pumping, we shine circularly polarized laser light on the alkali atoms, inducing a common spin orientation on a relatively large portion of the alkali atoms and, to a lesser extent, on the xenon atoms as well.

\section{Alkali Precession and Magnetometer Signal}

The immediate goal of optical pumping here is to achieve coherent Larmor precession among the alkali population for magnetometer operation. Recall that Larmor precession occurs when the atomic spin vector is offset from the direction of the net magnetic field. Also recall that the magnetic field defines the possible spin energy states and the quantization axis along which the atoms get polarized by optical pumping. Now, if we introduce a small transverse DC field, say along the $x$ axis (which is coming out of the page in Figure 4), thereby shifting the net DC field to be pointed slightly off from $z$, we 
also shift the quantization axis. If this were the whole picture, the atoms with spin along $z$ at the instant the transverse field were applied would precess about the new net field, but not for long. Even though the pump laser still propagates directly along $z$, any atoms that get polarized by it in the presence of this transverse field will have quantized spin along the new axis of the net DC field and not perfectly along the $z$ axis. Figure 15 illustrates this concept.

So, in order to initiate alkali precession, the field direction must change appropriately, but when it does the coherent precession decays as the atoms get repolarized along the new field direction, or collide with other atoms and lose their spins, until the coherent precession is once again immeasurable. In other words, to maintain coherent alkali precession, the field direction must change at specific intervals within the alkali spin lifetime.

Now, recall that we can also have an AC magnetic field on $z$. This is the key for magnetometer operation: since the net DC field is now offset from $z$ in the presence of the small transverse DC field, the $z$-AC field looks to have a transverse component relative to the spin-polarized alkali atoms, as shown on the right in Figure 15 below. 

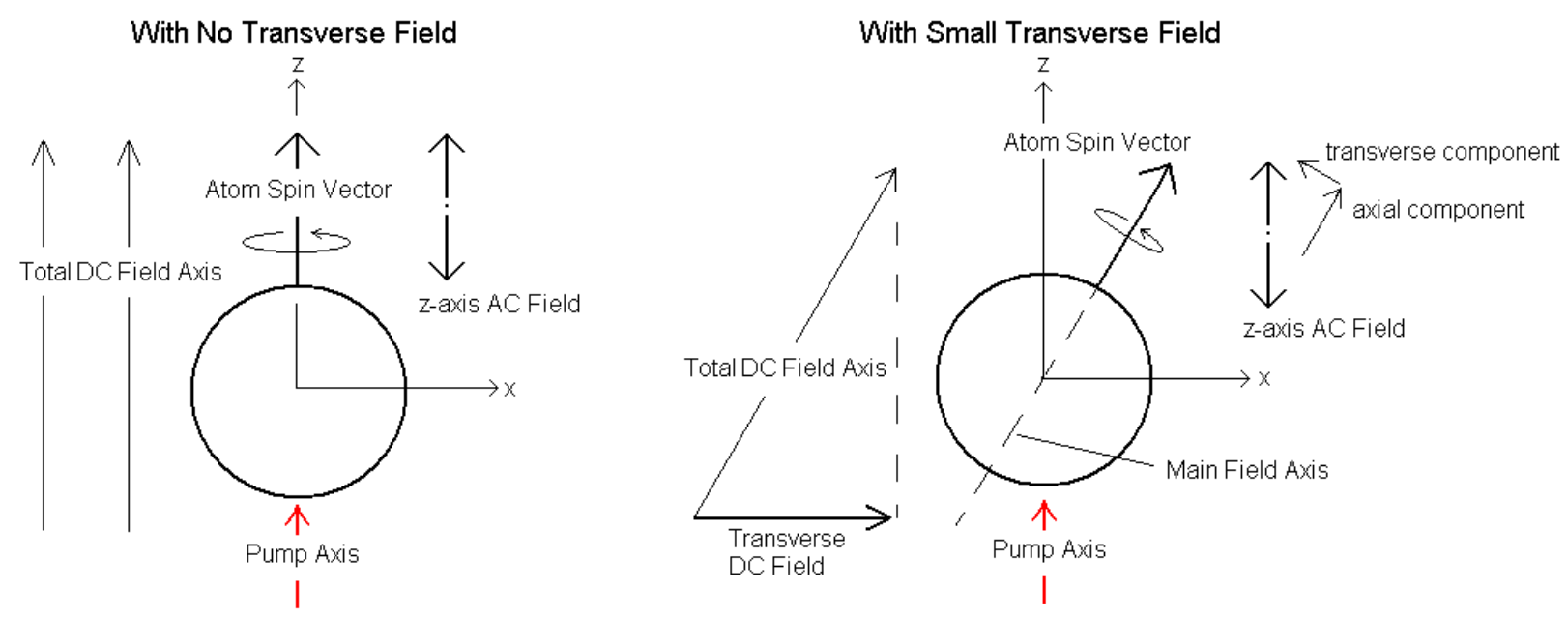

Figure 15. Introduction of a small transverse DC field offsets the total DC field and the polarized alkali spin vector from the $z$ axis. The $z$-axis $\mathrm{AC}$ field then has a transverse component relative to the atom spin vector, which stimulates the alkali Larmor precession. Note that the illustrated transverse DC field here is extremely large for the purpose of demonstration.

The combination of the net DC field and the axial component of the AC field will henceforth be referred to as the main magnetic field. The transverse component of the AC field is by definition orthogonal to the atom spin vector, so the polarized alkali atoms precess about it. As soon as this precession begins, the atom spin vector is offset from the main field axis and so begins to precess about that, as shown in Figure 16 below. Of course at any given time, the atomic group is really only precessing about one axis - the direction of the total instantaneous magnetic field, including all the DC and AC fields but this process of stimulation is easier to visualize in components. 

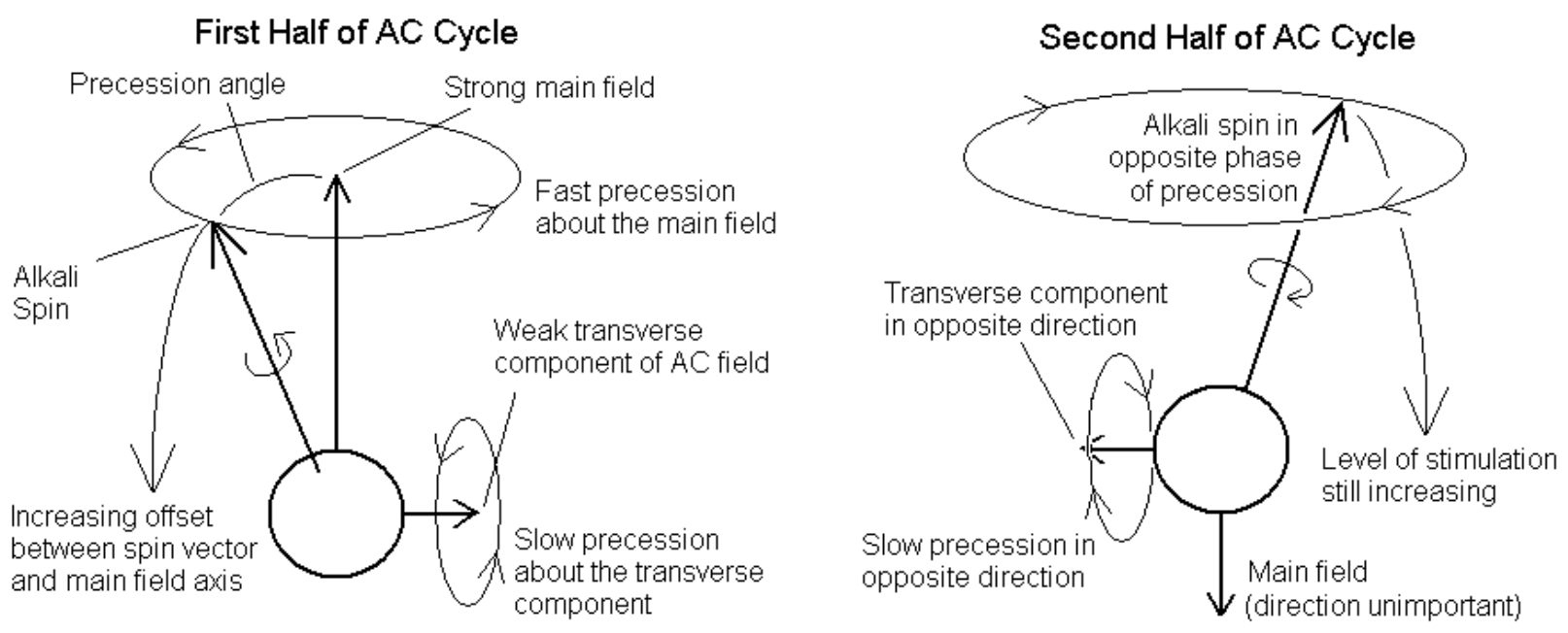

Figure 16. Component description of alkali stimulation process. The spin-polarized alkali atom begins to precess slowly about the transverse component of the $z$-AC field. This offsets the alkali spin vector from the axis of the main field (the sum of the net DC field and the axial component of the $z$ $\mathrm{AC}$ field) and thus initiates fast precession about the main field axis simultaneously. If the $z$-AC field alternates at the proper frequency, it will increase the precession angle (the level of stimulation) through each cycle until the atom loses its spin.

The level of stimulation (the precession angle), which defines the signal strength as we'll soon discuss, accumulates in magnitude from cycle to cycle. A single atom will continue to precess until it gets repumped or loses its spin in a collision; in order to increase the precession angle throughout the entire AC cycle and thus maximize signal, the field changes should occur at opposite phases of the atomic precession, as in Figure 16. This requires very precise tuning of the $z$-DC field strength and the $z$-AC field amplitude and frequency. If done properly, each field change stimulates the newly polarized atoms while continuing to increase the precession angle of the whole group. After enough time has passed, a portion of the alkali group will have been pulled into coherent precession about the main field, and the total atomic group reaches a steady state when the rate of 
signal increase from the growing precession angle equals in magnitude the rate of signal loss from atomic collisions and repolarization.

We can see that, up to a certain magnitude, a larger transverse DC field will offset the main field farther from the $z$ axis, resulting in a larger transverse component of the $z$-AC field relative to the alkali spin vector. This increases the rate of stimulation, which also means that on average more signal is lost each time an atom loses its spin, but overall the result is a larger average precession angle among the coherent atoms at steady state and thus larger signal. In short, a stronger transverse magnetic field generates a stronger signal, which is exactly what we want in a magnetometer. The alkali lifetime in our setup is generally on the order of tens of microseconds, so the process reaches steady state relatively quickly, giving the magnetometer a nice, fast response time as well.

It is also important to recognize the significant contribution from the axial component of the $z$-AC field to the main field (revisit Figure 15); whenever the $z$-AC field changes, the main field also changes, and quite dramatically at that. In most applications, the AC field alternates by a sine wave, but for the ease of demonstration we'll use a square wave here. Once the delicate balance of field parameters is achieved in this example, the main field source alternates between $-1 \mathrm{~V}$ and $+3.5 \mathrm{~V}$, resulting in the steady-state alkali signal shown in Figure 17 below. 


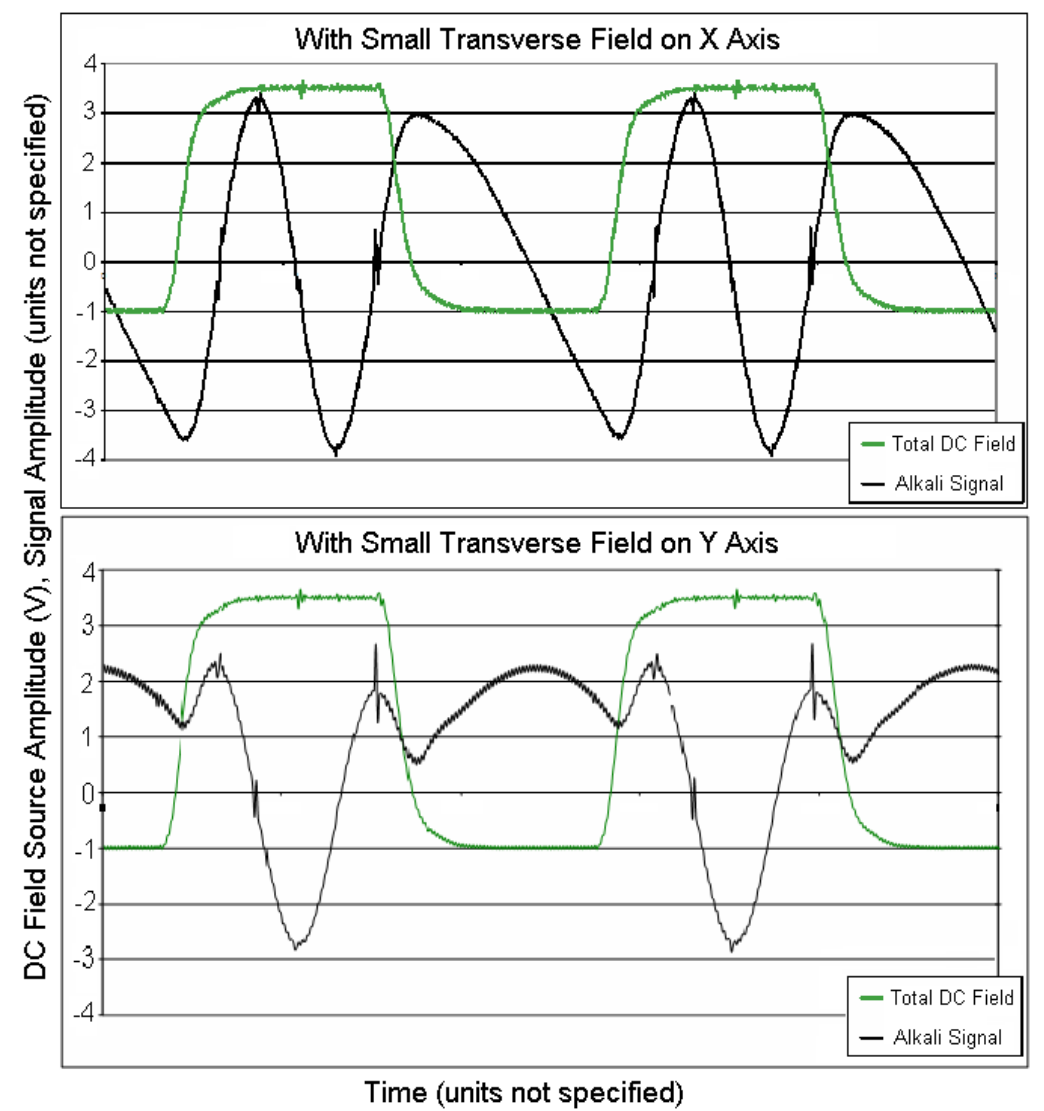

Figure 17. The magnetometer carrier signal generated when a transverse DC field is applied on the $x$ axis (top) and on the y axis (bottom). Scale units for the raw signal have been removed from the axes.

So, the main field alternates in direction and magnitude throughout the $z$-AC cycle, and since Larmor precession depends on the field, the alkali precession direction and frequency alternate with it. The alkali signal directly represents the behavior of the net alkali spin vector relative to the positive $y$ axis; we will discuss exactly how this works shortly. Looking closely, we can see the decay of the signal within each half-cycle of the field, as alkali atoms lose their spin and the signal decreases until the following field change occurs. 
More importantly, notice that the alkali precession quickly goes through one and a half revolutions in one direction while the main field is positive and large, and then slowly backtracks half a revolution in the reverse direction while the field is negative and small. This setup accomplishes three crucial things: first, the $z$-AC field reverses direction at the appropriate times of the atomic precession, so the precession angle continues to grow through each cycle of the AC field, as in Figure 16, until the angle is proportional to the field strength; second, one cycle of the AC field results in one full revolution of the alkali precession, which has been shown to maximize the magnetic resonance amplitude to produce the cleanest magnetometer carrier signal; third, applying the small transverse DC field on $y$ instead of $x$ shifts the initial phase of the precession by 90 degrees, and since there are two stages of precession in the AC cycle this dramatically changes the alkali signal waveform as seen in Figure 17. In other words, fields on $x$ produce a different carrier signal than do fields on $y$, and the overall amplitudes of the two signals represent the strength of the transverse field along the respective axes, so we can demodulate the two signals independently and resolve the actual transverse magnetic field vector over time.

So there we have our two-axis vector magnetometer, which measures the strength and direction of magnetic fields in the $x-y$ plane of the device. Of course, when trying to measure too strong a transverse field, the transverse component of the $z$-AC field can actually rotate the alkali spins so quickly that they overshoot 90 degrees off $z$ before they decay; at that point the signal cannot get any bigger and we say the magnetometer is saturated, which essentially means it is outside its functional limit. However, for gyro 
applications this is almost never a concern because the fields generated by the xenon atoms in the cell are within the linear response region of the magnetometer.

We now understand how the alkali population responds to transverse magnetic fields to serve as a magnetometer. What we have not yet discussed is how we actually measure a signal from the alkali spin. To do so, we employ a method known as Faraday detection, which utilizes a physical phenomenon called Faraday rotation.

\section{a. Faraday Detection}

In 1845, before Maxwell proved that light and EM energy are actually the same thing, Michael Faraday discovered an interesting interaction between a light ray traveling through a dielectric medium and an external magnetic field applied across the medium. He noticed that if light enters the medium linearly polarized along some arbitrary axis, it exits the other side of the medium polarized along a different axis. He deduced that the magnetic field rotates the polarization axis as the light propagates through it, as shown in Figure 18, calling this effect Faraday rotation. The stronger the field, the faster it rotates the polarization axis. ${ }^{3}$ 


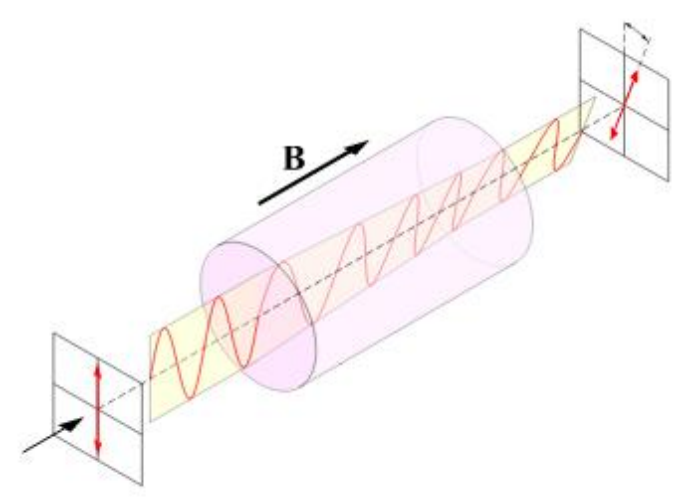

Figure 18. Faraday rotation. Light polarized along an arbitrary axis propagates through a dielectric material with a relatively strong, externally applied parallel magnetic field $B$, experiencing a rotation of the polarization axis.

It is difficult to accurately describe Faraday rotation without a rigorous mathematical approach. For our purposes, the alkali atoms in coherent precession serve as the magnetized medium, where the magnetic field is generated by the spin of the charged atoms. Therefore, as the atoms precess, the magnetic field through the medium changes directions and causes the polarization axis of the transmitted light to fluctuate.

Imagine the precession of the coherent alkali group as the sense laser light passes through the vapor cell. The laser is linearly polarized, roughly along the $\mathrm{z}$ axis; for now let's just arbitrarily call it the vertical axis. Recall from Figure 8 that the $\pi^{0}$ polarization axis can be expressed in terms of the relative phase between two imaginary photons $\sigma^{+}$and $\sigma^{-}$. As shown in Figure 19 below, if a $\pi^{0}$ photon from our sense laser passes through the cell while the alkali spin vector opposes the photon direction of propagation, then the vapor will retard the imaginary $\sigma^{+}$and advance the $\sigma^{-}$. This results in a relative phase offset 
between the two, which translates to a rotation of the actual $\pi^{0}$ polarization axis. During the other half of the alkali precession cycle, the effect is reversed.
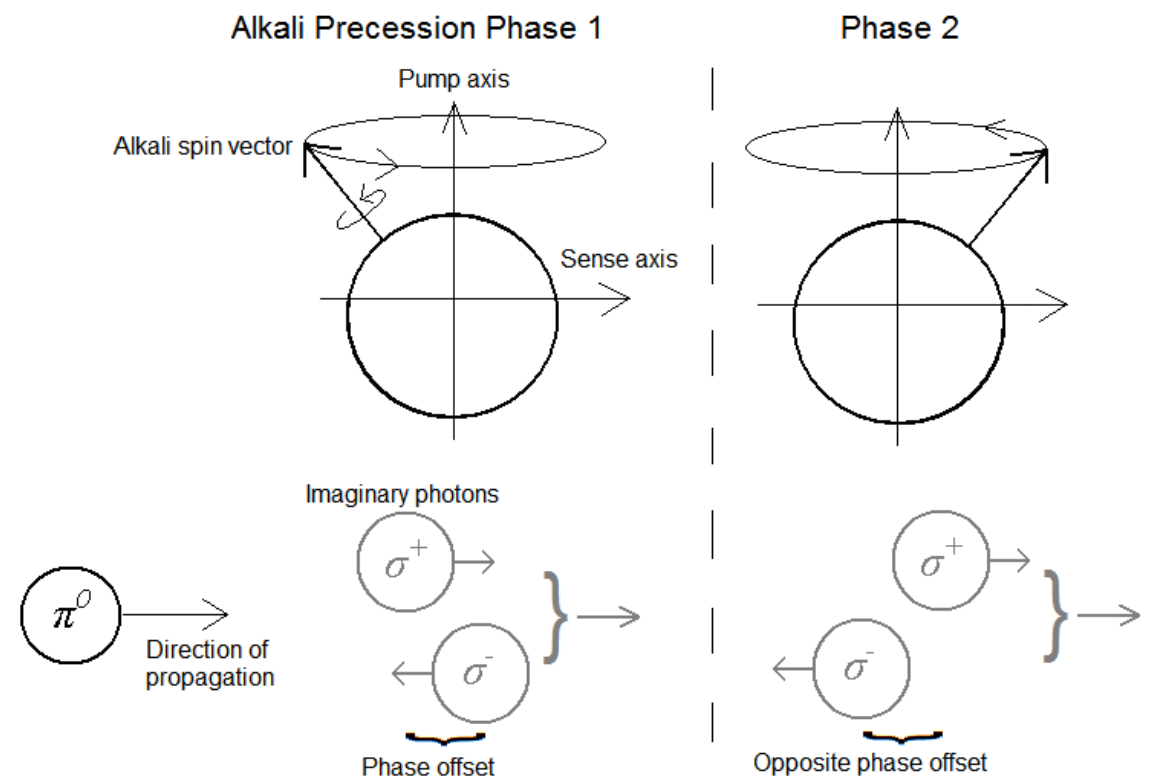

Figure 19. Faraday effect dependence on alkali precession phase. During one half of the precession cycle, the alkali spin opposes the angular momentum of one of the imaginary circularly polarized photons and supports the other, causing a relative phase offset and thus altering the linear polarization axis.

The sense light that is transmitted through the cell passes through a polarizing beam splitter. The two resulting component beams are sent to photo-detectors on the Hobbs circuit, which finds the difference in their intensities to determine the polarization axis. We can see that over time, the offset of the polarization axis, taking vertical as zero, would trace out a sine wave (for this simple example) with frequency equal to the Larmor frequency of the alkali, as in Figure 20. 
Thus we have a carrier signal directly correlated to the alkali precession. Also, a stronger transverse field leads to wider Larmor precession, causing a larger Faraday rotation and ultimately stronger signals. This is just what we want from a magnetometer.

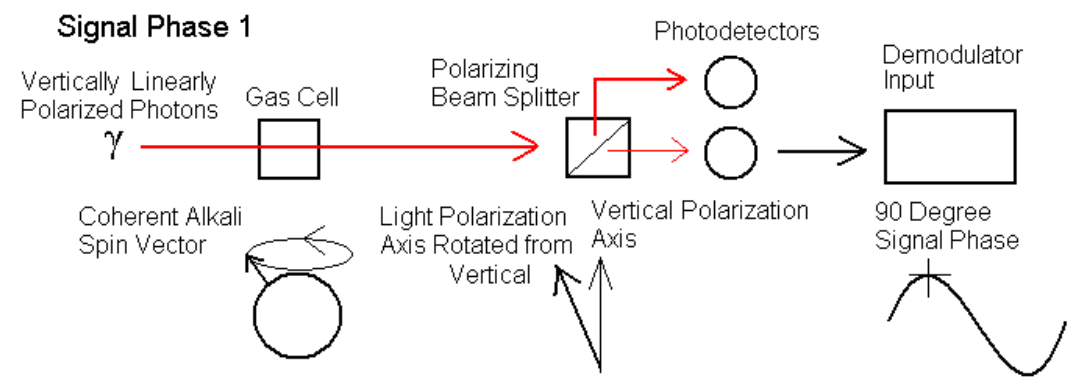

Signal Phase 2

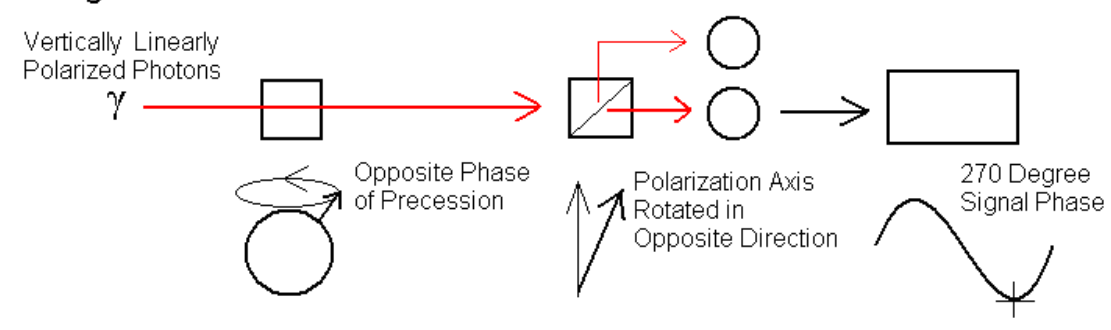

Figure 20. Visual representation of Farady detection setup. The difference in intensities on the two photodetectors indicates the polarization axis of sense light transmitted through the cell, which fluctuates with the precessing alkali atoms, producing the magnetometer carrier signal.

\section{Xenon Precession and the NMR Gyroscope Signal}

So we have a magnetometer that can detect small changes in magnetic fields transverse to the $z$ axis. Recall that our vapor cells contain not only alkali, which is central to magnetometer operation, but ${ }^{129} \mathrm{Xe}$ and ${ }^{131} \mathrm{Xe}$ as well. Recall too that some of the xenon atoms are polarized through spin exchange with the pumped alkali atoms. Finally, recall that there is a steady DC magnetic field on $z$ (the alternating field on $z$ operates at around 
30 to $80 \mathrm{kHz}$, close to 1000 times the Larmor frequency of either xenon isotope, so it has very little effect on the DC field experienced by xenon). All of this means that the polarized xenon atoms can also have coherent Larmor precession about the $z$ axis. What's more, our magnetometer can pick up on the transverse magnetic fields generated by the coherent xenon spins and therefore track the xenon precession.

All we need is a way to initiate the xenon precession. We can calculate the expected Larmor frequency for our setup using equation $\mathrm{i}$, and as discussed we can inject a magnetic field on $x$ while picking up very little of it on $y$. So driving the $x$ coil with a sine wave of the xenon Larmor frequency will stimulate the xenon precession, just like the component of the $z-\mathrm{AC}$ field transverse to the alkali spin stimulated the alkali precession in in Figure 16.

So now we have some population of xenon atoms with coherent precession about $z$ at a known, steady frequency. The magnetometer picks up the transverse magnetic fields generated by the xenon (recall that the magnetometer can measure the full, two-axis transverse field vector by the separate $x$ and $y$ carrier signals at any time). Now, the electronics establish a reference signal synchronized to the ${ }^{129} \mathrm{Xe}$ precession when the gyro is stationary in inertial space so that, if everything is left untouched, the reference signal and the actual magnetometer signal from the coherent xenon precession are identical. However, any physical rotation of the system about $z$ alters the sense axis without disturbing the coherent precession of the xenon atoms. This appears as a phase 
shift between the reference and the actual signal, where the phase difference between the two is a direct measurement of the physical rotation of the unit, as shown in Figure 21.

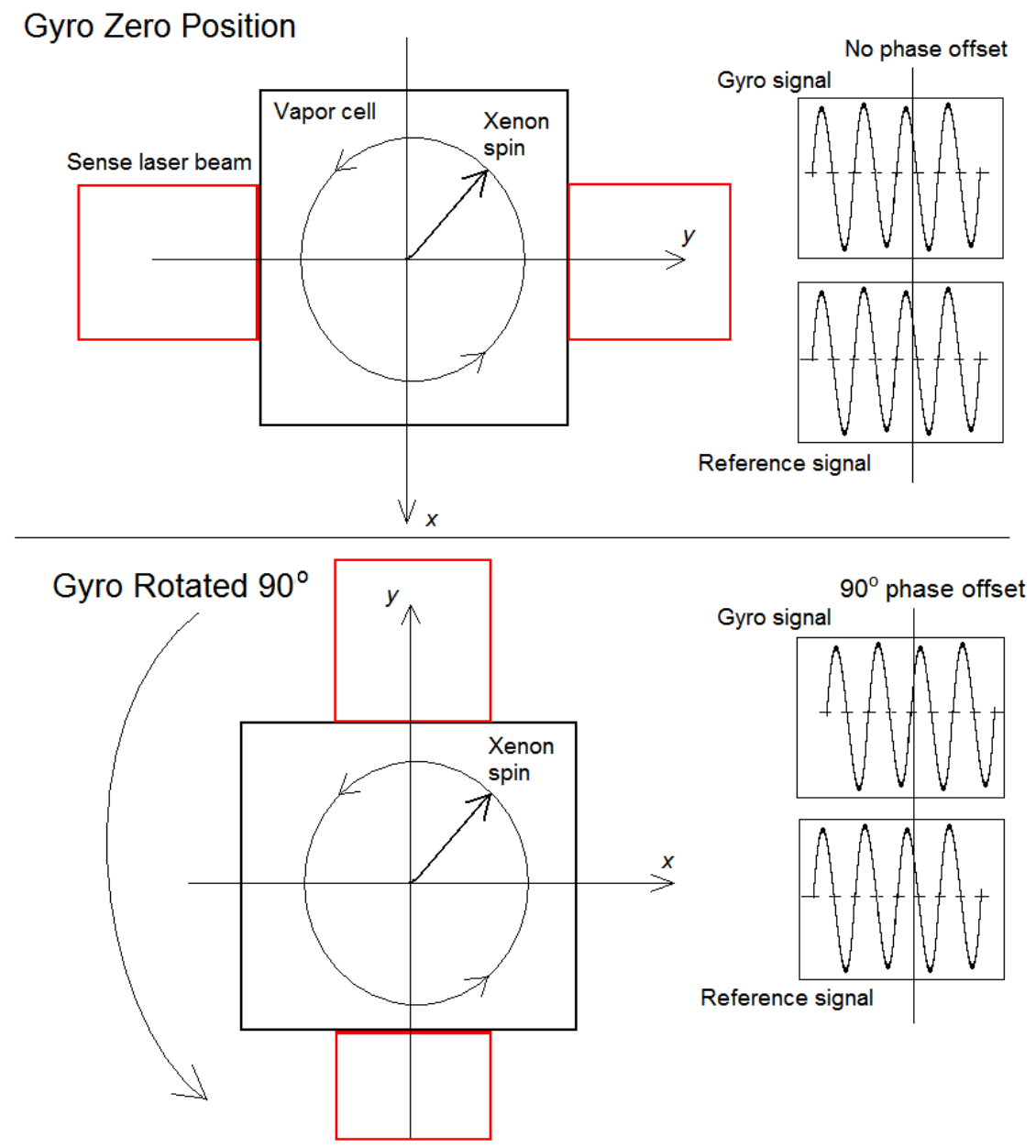

Figure 21. Basic concept of operation of the NMR gyroscope. As the gyro is turned about the $z$ axis, a phase offset is introduced between the gyro signal and the reference to the original xenon precession. The phase offset directly measures the physical rotation of the device, giving us an NMR gyroscope.

To summarize the main elements of the system, the pump laser prepares the alkali vapor, dumping a disproportionately high population of the atoms into the $F=4, m_{F}=4$ state, thereby aligning their spins. The alkali atoms interact with the xenon atoms, exchanging 
spin and resulting in a higher population of aligned xenon. A small-amplitude sine wave is injected on the $x$-axis coils to generate a magnetic field to stimulate the xenon precession about the $z$ axis. The transverse component of the xenon spin offsets the total field from $z$, causing the alkali to precess. The alkali behavior is observed using Faraday detection, and by demodulating the alkali carrier signal for fields on $x$ and $y$ we can track the xenon precession and compare it to our reference for gyroscopic sensitivity. Extremely small changes in magnetic fields allow this gyro to function, so for it to even be useful (let alone a breakthrough technology) the fields must be extremely stable to keep high levels of precision.

Two characteristics are particularly important when it comes to the precision of the device. The first and most obvious is the signal to noise ratio (SNR). As with any sensor, a single measurement will not be exactly correct; random, uncontrollable, and unpredictable influences on the signal constantly distort the output, causing deviations from the true quantity the sensor is supposed to measure (in our case, angular rotation). If a gyroscope is held steady in one position for a long time, its orientation can be determined to high accuracy by averaging the measurements made while in that one position, but of course that limits the response time of the device. Any single measurement will have a relatively high error associated with it. Every single component of the system pays some contribution to SNR, but the most important improvements are those made to the fundamental noise limits, which usually stem from cell filling and sealing procedures (which is why measuring internal pressures is important). 
Let's use the analogy of a coin being flipped. Suppose a hundred people stand side-byside along a thin line painted on the ground which represents the true angular rotation to be measured. Each person flips a coin; if a person's coin reads heads, they take a step forward and if it reads tails they step back. The result after they have all flipped their coins would be a random scatter with roughly half the people in front of the line and the other half behind it. This scenario represents the SNR metric; even though the average final position of all one hundred people would be very close to right on the line, the position of any one person would be relatively far off from that line.

Another common metric for gyro performance is something called angle random walk (ARW). Return to our coin analogy, but suppose instead there is only one person. This person starts on the line and flips a hundred coins, taking a step forward for every heads and a step back for every tails; this is a literal random walk. After a hundred flips, this person may end up right back on the line, but more likely the person will stand some number of steps in front or behind the line. This represents ARW; the total error (distance from the line) for any single measurement (coin flip) is dependent upon the accumulated error from all the previous measurements. The ARW tends to add noise at a constant rate relative to the square root of the number of measurements, so if the measurements are made consistently in time, ARW is expressed in degrees per the square root of time that measurements have been recorded.

Both SNR and AWR are highly affected by the lifetime of the coherent xenon precession. If we remove the drive stimulating xenon precession, we want to know how long the 
xenon precession about $z$ lasts, or in other words how quickly the signal it generates diminishes. As we discussed for the alkali, the spin state of a polarized atom only remains for a brief time before collisions with other gases or the cell walls destroy the spin state; the lifetime is the amount of time it takes for a certain portion of the atoms to lose their spins.

It seems somewhat intuitive that a longer transverse spin lifetime should mean higher stability, but why does the lifetime even matter if the stimulating drive is always running? Well, first of all the lifetime affects SNR. The longer the atoms go on average without losing their spins, the stronger their net transverse projection gets and therefore the stronger the net signal they produce (for an injected drive of fixed strength). That right there is a big part of it, but there's more. With shorter coherence lifetimes, the population of precessing xenon atoms spreads out in phase more quickly, leaving a larger uncertainty in the net spin and thus less precision for any measurement.

Since ARW accumulates from measurement to measurement, reducing the precision even slightly can increase ARW significantly, which is why it is so important to maximize the spin coherence lifetime. Figure 22 below shows how SNR and the transverse spin lifetime (denoted T2) play into ARW for an NMR gyro. ${ }^{6}$ In order to make this simple relationship work, the SNR must be expressed in decibels divided by the square root of the correlation time (the time between measurements), which for our setup is one fourth of the Larmor precession period. 


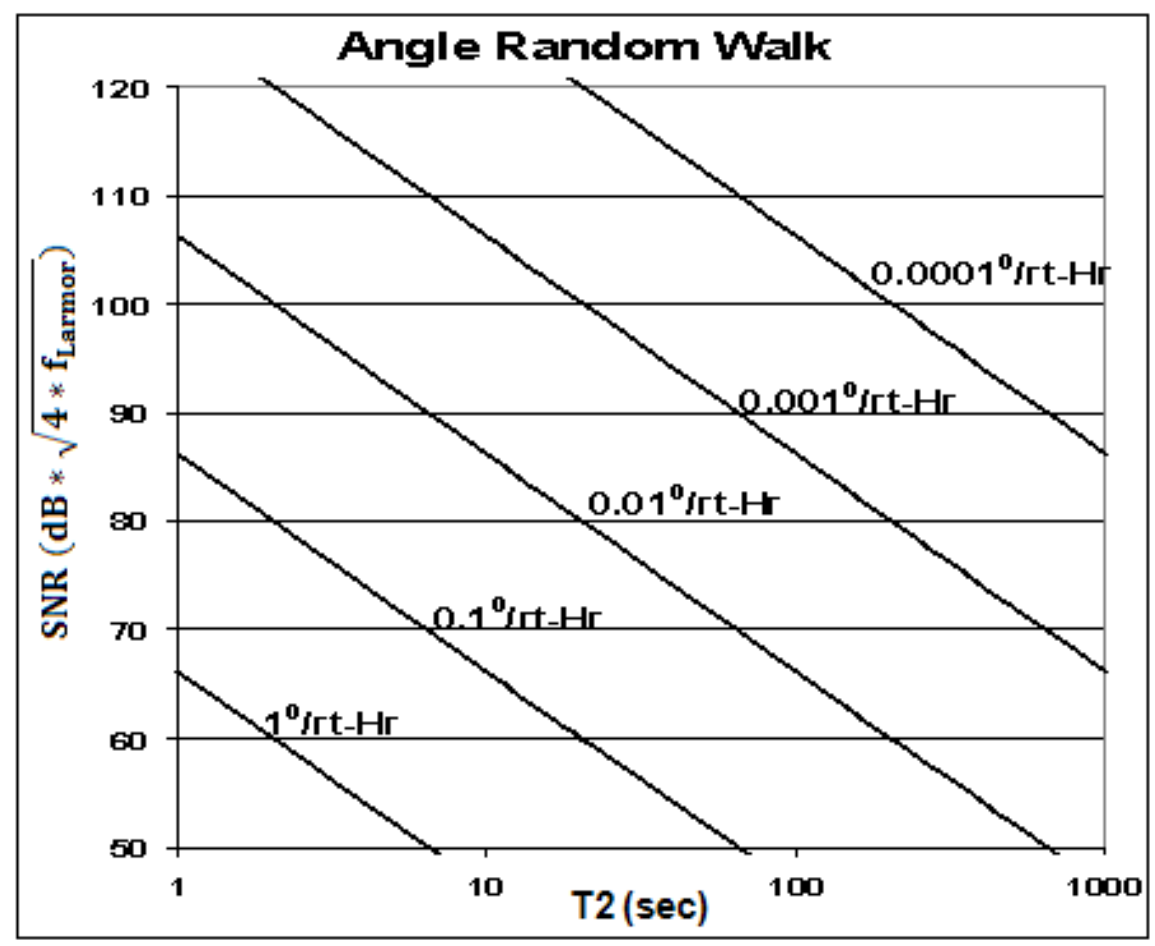

Figure 22. Angle random walk as it depends on the signal to noise ratio and the longitudinal spin lifetime T2 for an NMR gyroscope. To limit ARW, a vapor cell must exhibit both high SNR and long T2 values.

Also, if our stimulating drive drifts to something close to but not exactly at the natural xenon resonance frequency, the xenon atoms will slowly get pulled into precessing at the drive frequency (with a smaller net spin projection). In cells with shorter xenon lifetimes, this shift in precession frequency progresses more quickly. Even if we manage to drive exactly at the natural frequency, small noise deviations in the drive will alter the spin group, which appears as a phase shift from the reference signal and thus causes false gyroscopic readings. These temporary deviations in the drive frequency will clearly have a large impact on cells with short spin lifetimes, but for cells with sufficiently long lifetimes, such shifts are not likely to impact the gyro signal significantly. This is why long spin lifetimes are so important for gyro performance. 
So the quality of the NMR gyroscope is heavily dependent upon the transverse spin lifetime, which means we want to build our gas cells in a way that allows for as high a lifetime as possible for both ${ }^{129} \mathrm{Xe}$ and ${ }^{131} \mathrm{Xe}$. This goal is much easier said than done. The science of cell construction for atomic applications remains a very active field of research and is far from well understood. Enough progress has been made for preliminary gyro operation, but if this technology is going to challenge the boundaries of current navigation capabilities, we'll need further advancements.

Now, given what we have discussed thus far, we have a good understanding of the fundamental physical principles that drive the NMR gyro operation. From this point on, we will focus our discussion on a very specific topic within the overall goal of improving the gyro. We will cover some of the most significant factors that affect nuclear spin lifetimes and our current efforts to utilize those factors to our advantage. We will examine the techniques by which we currently measure the lifetimes (our industry standard techniques), and I will also introduce several other approaches that had not previously been used in our setup. Lastly, I will summarize my work on implementing these other techniques in our system, including the hardware and software modifications necessary to make them work. We will compare results from the new and old measurement techniques to verify my work, and we will also go over various factors that make the new techniques superior. My goal is to provide a useful catalog of tips, guidelines, pitfalls, and general explanations for a number of approaches to NMR lifetime measurements. So first off, let's talk about the nuclear spin lifetime itself, its general 
behavior, and the factors that make the difference between a lifetime of 3 seconds and 30 seconds.

\section{B. Lifetimes of Nuclear Spin States}

Imagine the system running in its normal conditions, as we've been discussing. If the transverse field that drives the xenon precession suddenly shuts off, the signal weakens as more and more xenon atoms lose their coherent precession. The atomic signal strength usually decreases by an exponential decay. The half-life of the atomic spin is the amount of time it takes for $1 / 2$ of the coherent population to lose their spins. Similarly, the lifetime is the time it takes to reach $1 / e$ of the original coherent population, where $e$, sometimes called Euler's number, is the irrational constant whose exponential form $e^{x}$ is its own derivative; the value of $e$, truncated to five decimal places, is 2.71828 . So the lifetime in this case is a measure of how long it takes for the signal to diminish to just over $1 / 3$ of its original amplitude.

There are two xenon spin lifetimes of great importance to us; $T 1$ is the longitudinal spin lifetime and $T 2$ is the transverse spin lifetime. T1 is a measure of the longevity of the $z$ axis polarization, and $\mathrm{T} 2$ is essentially a measure of how long the polarized group maintains coherent precession (with transverse spin projection components in equal phase) about $z$. As such, T1 is the upper limit for T2 since coherent precession is impossible without net longitudinal polarization. With perfect magnetic field uniformity 
across the cell, T2 should be equal to T1, but any non-uniformities decrease T2 from the T1 value. See, if the cell contains slightly different magnetic fields in some parts of the cell than others, the precession frequency of the atoms changes as they pass through these parts of the cell. Also, the atoms do not completely uniformly sample the magnetic field in the cell (since the motion of an atom through the buffer gasses is random in direction and path), so we cannot hope for these small changes in frequency to average out completely. During the time that the precession frequency of an atom differs from the average of the group, it accumulates a phase offset from the coherent group. Eventually many atoms have large enough phase offsets that we can no longer distinguish a signal. So even though a good portion of the xenon population is still polarized along $z$ and even still experiencing precession about $z$, the precession of the group is no longer in coherent phase and so $\mathrm{T} 2$ is shorter than $\mathrm{T} 1$.

Therefore, even though the transverse lifetime $\mathrm{T} 2$ is the parameter that truly limits the gyro's performance, we are often more interested in measuring the longitudinal spin lifetime T1 during cell testing because it limits T2. In fact, we rarely concern ourselves with the actual T2 value during cell testing because we can achieve very high levels of magnetic field uniformity with some effort once a cell with long T1 times for both ${ }^{129} \mathrm{Xe}$ and ${ }^{131} \mathrm{Xe}$ is found. Even though our ultimate goal is to maximize T2, we focus on increasing T1 which in turn allows for longer T2 times, so let's discuss our current efforts to maximize T1 lifetimes. 


\section{The Major Influences on Longitudinal Spin Lifetimes}

As previously discussed, the only things that really dictate spin state lifetimes are xenon collisions with the cell walls and other gas atoms in the cell. The best we can do is to surround the xenon atoms with atoms of buffer gases whose spin-exchange interactions with xenon are very low. Nitrogen makes a good buffer gas for this purpose. To reduce the effect of the walls, the cells are prepared with a small amount of hydrogen as well. Ideally a thin layer of cesium-hydride or rubidium-hydride, both of which have a much lower chance of destroying the xenon spin than does the bare glass of the cell, forms across each inner face of the cell. It also seems advantageous to allow the cell to cure at around 100 degrees Celsius for a day or two to allow ample formation of the hydride. As far as physical construction of the cell is concerned, it basically comes down to mixing these gases properly and going to great lengths to clean the glass cells of all contaminants before filling and sealing them.

However, there are other parameters that affect the collision rate inside the cell. The size of the cell, for example, determines the wall collision rate; an atom in a smaller cell has a shorter mean path between walls and thus spin lifetimes are shortened by more frequent wall collisions. As an aside, we often test new cell construction methods on large spherical cells, not only because they are easier to construct but also because they allow for longer lifetimes and stronger signal from the increased number of alkali atoms, so the effects of different cleaning and filling methods are more apparent. Once we find an approach that seems to lead to consistently long lifetimes, we move on to repeating the 
process on smaller cubic cells. These inherently have shorter lifetimes and smaller signal potentials and are thus harder to work with, but they are necessary to meet the specific requirements of the gyro.

On the other side of the coin, we can alter alkali-xenon collision rate by changing the cell test temperature. With decreased temperatures come decreased alkali pressures and lower particle velocities, reducing collision rates and thus extending spin lifetimes, especially for ${ }^{129} \mathrm{Xe}$. Figure 23 shows an extreme case of this effect measured in somewhat of a fluke of a cell.

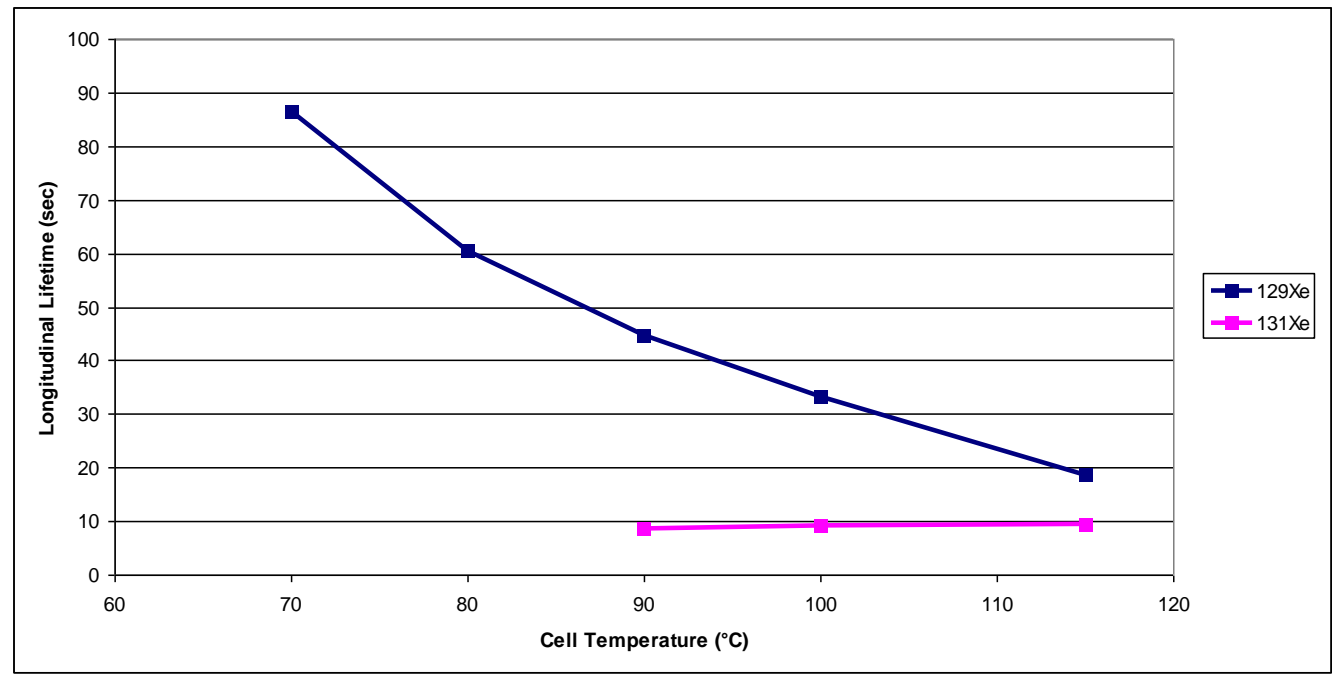

Figure 23. The effect of cell temperature on longitudinal spin lifetimes for both xenon isotopes. This cell has abnormally low wall dependence for the ${ }^{129} \mathrm{Xe}$ lifetime.

Clearly, temperature has a huge effect on the $\mathrm{T} 1$ for ${ }^{129} \mathrm{Xe}$, but almost no effect for ${ }^{131} \mathrm{Xe}$ because at sufficiently low temperatures, the wall collision rate becomes the dominant factor and further temperature reduction does not extend the lifetime; ${ }^{131} \mathrm{Xe}$ lifetimes are 
much more dependent on wall conditions for reasons we will discuss later. The reason that the ${ }^{131} \mathrm{Xe} \mathrm{T} 1$ actually decreases with temperature is that lower temperatures mean lower particle velocities, meaning xenon atoms spend more time in contact with the cell walls during collisions, or in other words the duration of adsorption increases. So when the lifetime is wall-collision limited, we actually tend see lower lifetimes with lower temperatures.

Measuring T1 at different temperatures is in fact an extremely useful cell testing procedure because it allows us to identify whether a given cell has T1 limited by wall collisions or gas collisions at our target test temperature of about 115 degrees Celsius. As in Figure 24, most cells exhibit wall-dominated lifetimes for both isotopes at higher temperatures compared to the example in Figure 23.

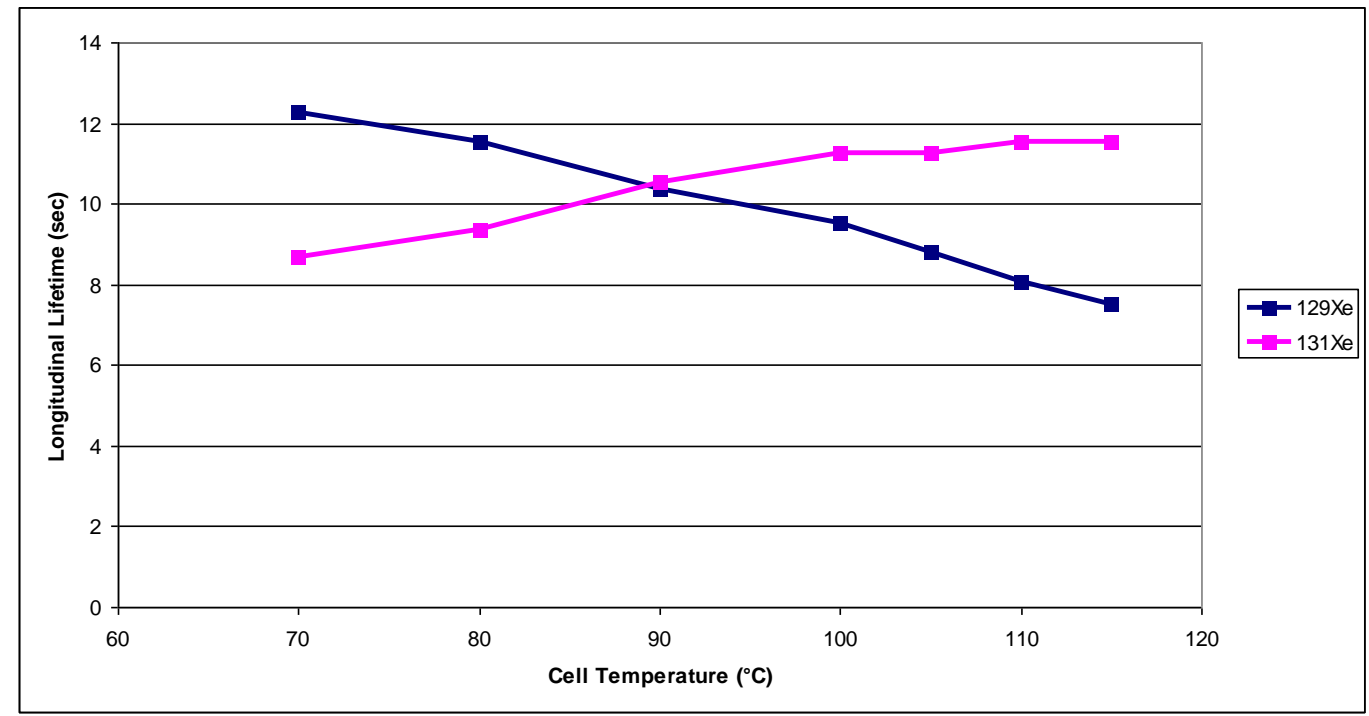

Figure 24. Typical trend for longitudinal lifetimes over cell temperature. As gas collision rates decrease, wall effects become the dominant limit to $\mathrm{T} 1$. 
Notice that this cell would hit a T1 limit of 13 to 14 seconds for ${ }^{129} \mathrm{Xe}$. This type of test tells us whether our efforts to improve wall conditions are working. For ${ }^{129} \mathrm{Xe}$, the wall limit usually occurs at relatively low temperatures, whereas for ${ }^{131} \mathrm{Xe}$ the wall limit tends to be up around the target temperature of $115^{\circ} \mathrm{C}$. Our primary motivation in improving cell wall conditions is that the walls greatly affect the ${ }^{131} \mathrm{Xe}$ lifetime at the test temperature, whereas the ${ }^{129} \mathrm{Xe}$ lifetime is more dependent upon buffer gas conditions in that temperature range. Therefore, as stated before, our two main concerns in cell design are wall conditions and buffer gas mix.

Unfortunately, we cannot always perform the test over a wide range of temperatures because the signal strength is also highly dependent on cell temperature, given that it takes high temperatures to vaporize enough alkali to generate a strong magnetometer signal. Often the signal is not strong enough below 80 or $70{ }^{\circ} \mathrm{C}$ to make a reliable $\mathrm{T} 1$ measurement. Temperatures around $115^{\circ} \mathrm{C}$ tend to have the best balance between relatively high lifetimes and strong SNR, which is why we make that our primary test temperature and the target operating temperature inside the gyro.

Now that we are familiar with the general behavior of spin lifetimes and their contributing factors, we will move on to discussing the various ways we can actually make measurements of $\mathrm{T} 1$ and $\mathrm{T} 2$. We will begin with our industry standard techniques and then we'll get to the new techniques that have been set up to function on our system. 


\section{Techniques for Measuring Spin Lifetimes for NMR}

First off, it should be noted that these tests are performed on each isotope of xenon individually, so both T1 and T2 lifetimes must be measured twice for each cell. All of the techniques we will discuss involve stimulating the xenon precession using a transverse field, as discussed earlier. There are two ways we can accomplish this. For measurements that require an active drive throughout the test, we use a very low-amplitude sine wave of the xenon Larmor frequency from a function generator; for other methods we only need to get the xenon precession started and then we do not want any drive during the actual test. We accomplish the latter by a pulse drive, which is basically just a small number of cycles of a high-amplitude sine wave of the same frequency acting on the same coils, but the pulse is generated by the same computer that feeds the $z$-axis coils and records all the data (revisit Figure 4). We could also use the function generator and disconnect it at the start of the test, but there are reasons to prefer the pulse, as we will discuss.

One goal of the pulse is to get the xenon spins exactly orthogonal to $z$ to maximize signal (a $\pi / 2$ pulse, since it flips the spins $\pi / 2$ radians). The stronger the pulse is, the farther off $z$ it will push the spins until they actually overshoot 90 degrees; they can even be flipped completely to be anti-aligned with $z$ (a $\pi$ pulse), and this will be an important aspect of the pulse for our tests. Figure 25 below shows the basic concept of these two pulses. 


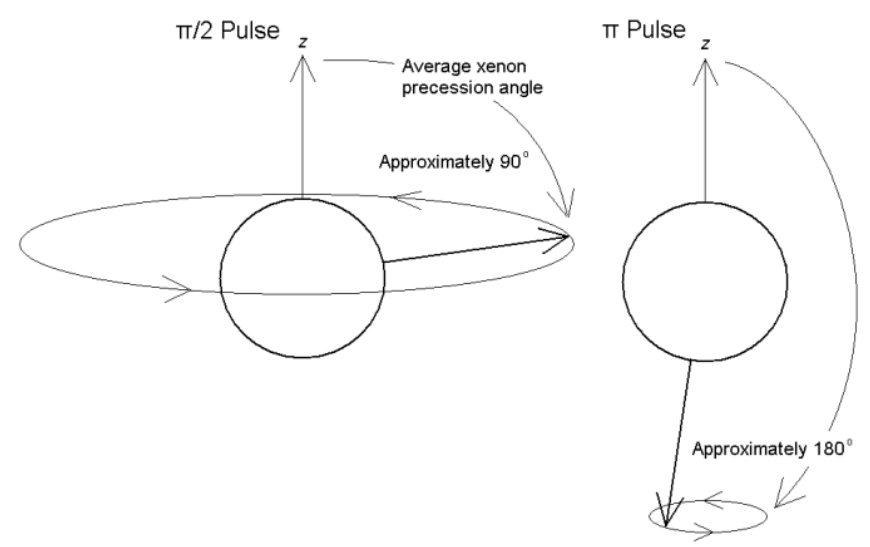

Figure 25. A $\pi / 2$ pulse is intended to maximize signal by pushing the total xenon precession orthogonal to $z$. A $\pi$ pulse is intended to reverse the polarization of the xenon spin, which results in very little signal (none if done perfectly). Unfortunately, it is extremely difficult to match both the frequency and amplitude needed to perform these pulses perfectly.

We can find the appropriate pulse strength by sweeping through pulses of various amplitudes and recording the initial amplitude of the xenon signal immediately following each pulse, as shown in Figure 26.

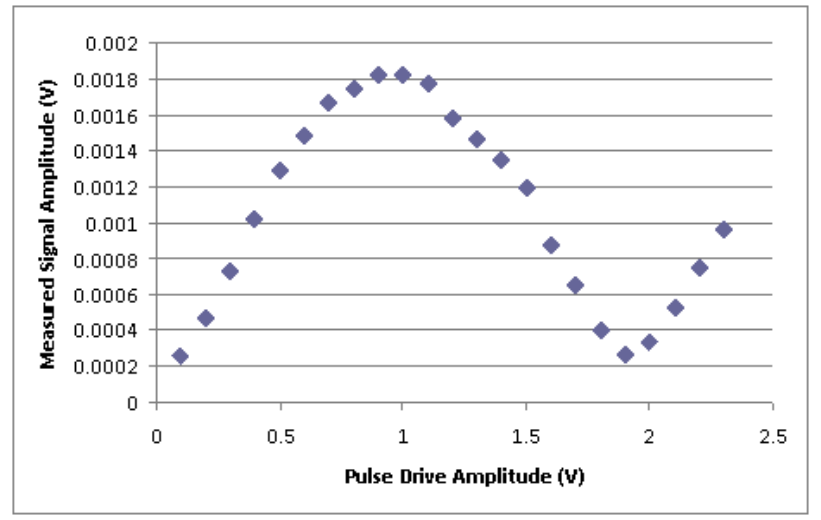

Figure 26. Relationship between signal size and pulse amplitude. The peak signal amplitude occurs at the pulse that drives the xenon spins 90 degrees off $z$ (a $\pi / 2$ pulse); the minimum signal (which should ideally be zero) occurs at 180 degrees (a $\pi$ pulse). Notice that pulses stronger than a $\pi$ pulse continue to push the spins past 180 degrees, increasing signals once again. 
Anytime the system is modified, it is useful to determine the pulse strength necessary for these two pulses. It is nice to hit the $\pi / 2$ pulse dead on because that maximizes signal, but it is even more important (and luckily easier) to determine the $\pi$ pulse; a zero is usually easier to find than a maximum, and the proximity to a true 180 -degree flip by a $\pi$ pulse determines the reliability of our T1 tests, so it is important to get close. Once we determine the amplitude for a $\pi$ pulse, we assume the $\pi / 2$ pulse to be half that amplitude.

Now, there are five basic techniques that we will focus on; two designed to measure T2 explicitly, two to measure T1, and one that measures both lifetimes simultaneously. We will first discuss the two industry standard methods that we perform daily (one for measuring T2 and the other for T1), detailing the experimental techniques and the procedures for analyzing data and extracting results. We will also cover some interesting and precautionary intrinsic properties of these two methods. We will then present three other methods, never before implemented on this equipment, and explain how we expect these methods to work. Following that will be a summary of my work attempting to implement these new methods and an exploration of the key parameters to focus on during setup to ensure that they give accurate results.

\section{A. Industry Standard Methods for Measuring Spin Lifetimes}

The simplest method of all, both in terms of procedure and the presumed physics behind it, is the free-induction decay method for measuring T2. 


\section{Measuring T2 Using the Free Induction Decay Method}

In this method, we use a $\pi / 2$ pulse to drive up the xenon precession and then allow the coherence to decay naturally by atomic collisions and by phase shifts from magnetic field non-uniformities. We record the signal as the atomic spins decay, ideally following a simple exponential decay curve. An example data set for a free induction decay (FID) test for ${ }^{129} \mathrm{Xe}$ T2 is shown below in Figure 27.

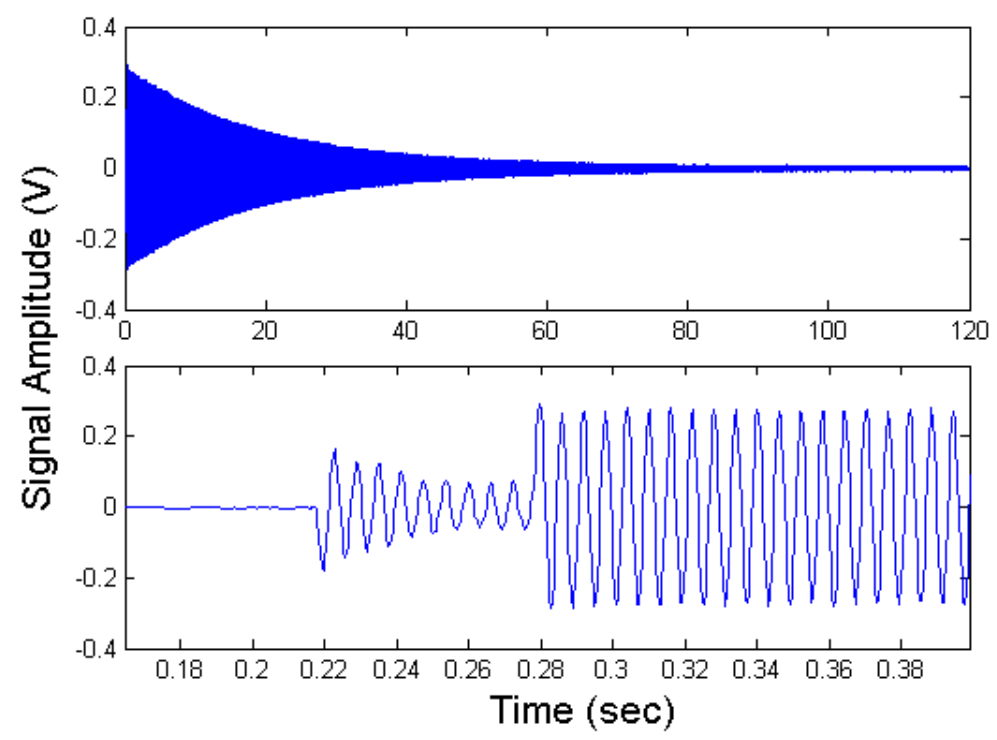

Figure 27. Raw signal following a $\pi / 2$ pulse for ${ }^{129} \mathrm{Xe}$. The bottom image shows a close-up of the data during which the pulse took place, where the xenon precession is growing up from the pulse, after which it is left to freely decay, as shown in the top image. This example exhibits good cross-axis rejection, as the pulse signature is smaller than that from the actual xenon precession.

Zooming in on the horizontal scale, we can see the results of the nine cycles of the $\pi / 2$ pulse as the signal grows up, and then when the pulse ends around 0.28 seconds, the 
actual magnetic sine wave coming from the xenon precession generates the decaying signal.

This is an appropriate time to discuss our method for reducing and analyzing data. The most direct way would be to determine the time at which the amplitude of the signal is $50 \%$ of the initial amplitude following the pulse (or from any point we deem the start, as long as we indeed have a simple exponential decay). We can easily calculate $\mathrm{T} 2$ from the half-life, which we'll discuss in more detail below. We could also fit the decaying sine wave with a theoretical curve and extract the lifetime from the fit solution. However, the raw signal can be influenced by many external factors; for either of these approaches, going merely by the shape of the raw signal allows for erroneous results. Still, we know that the signal is dominated by the sine wave of the xenon precession frequency, so we perform a Fast Fourier Transform (FFT) on everything following the pulse to convert our data from the time domain to the frequency domain, as shown in Figure 28 below. The FFT shows a tall, narrow, and isolated spike at the xenon resonance frequency, which means the precession frequency throughout the test was highly coherent (otherwise we would not be able to do NMR); we want to take down the value of the peak frequency, about $165.7127 \mathrm{~Hz}$. 


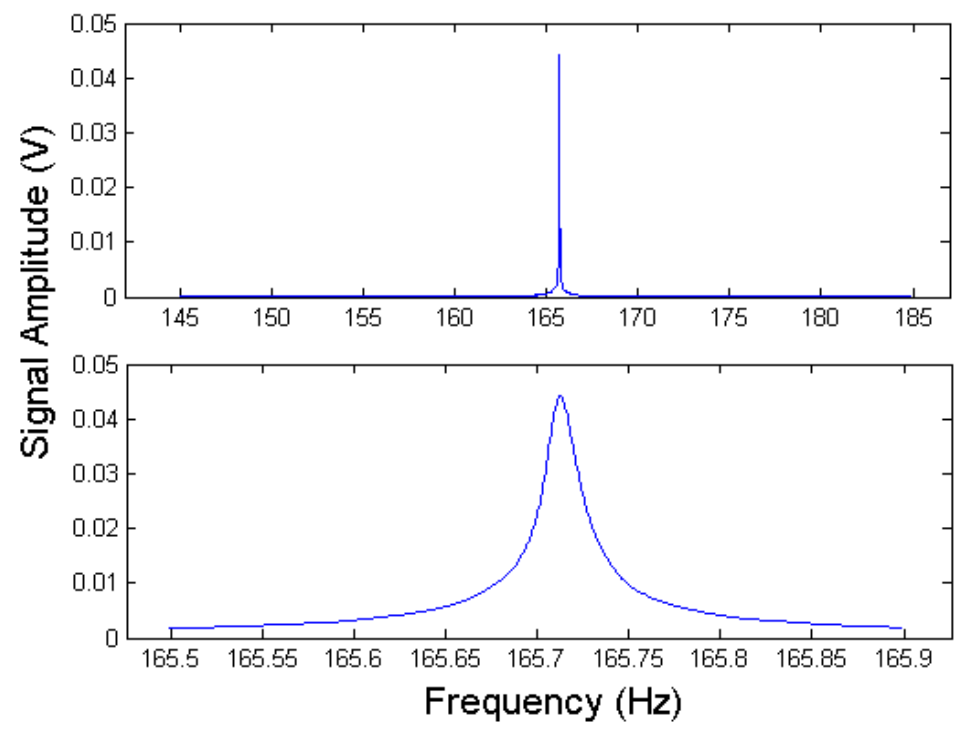

Figure 28. Signal from Figure 18 translated into the frequency domain using a Fast Fourier Transform (FFT). Clearly, the ${ }^{129}$ Xe resonance frequency is isolated and quite distinct. Zooming in, we can see just how well-defined the spike is, showing the central frequency to be around $\mathbf{1 6 5 . 7 1 2 7}$ Hz.

Now we go back though the data, performing an FFT on one small section at a time, say in windows of two seconds, and record the amplitude of each FFT at that frequency. We end up with a profile of our signal, as in Figure 29. We have not only eliminated any false shape in our signal from other-frequency sources, we also now can extract our lifetime from a simple decay curve and avoid dealing with the sine wave altogether (unless we have a particular reason to examine the raw sine wave). 


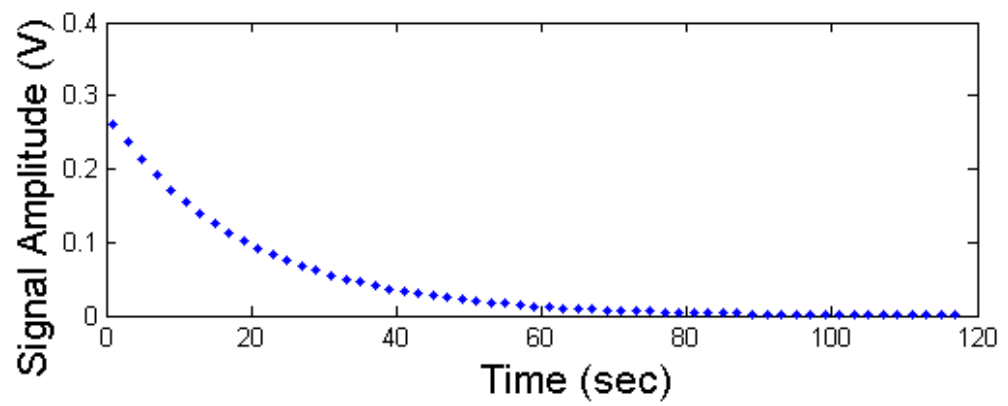

Figure 29. The signal profile created by performing an FFT on two-second windows of the raw signal and recording the amplitude of the FFT at $\mathbf{1 6 5 . 7 1 2 7} \mathrm{Hz}$ for each. The profile plots the FFT amplitude against the median time for each window.

If we reduce the window size, our curve will be more populated with data points, and we can get a close approximation of the half-life just by looking at the curve itself (though often decreasing the window size will increase the risk of shape defects in the curve, since the resolution of an FFT depends on the sample time used). The signal amplitude starts at $0.2741 \mathrm{~V}$ immediately following the pulse. We can look for the half-life, the time at which the signal reached $0.13705 \mathrm{~V}$ (half the initial amplitude), which occurred around 13.35 seconds.

Now, the formula to describe a simple exponential decay is

$$
y=A e^{\wedge}(-t / \tau)+v
$$

where $A$ is the initial amplitude, $t$ is time, and $\tau$ is the time constant, which for our purposes will always be the spin lifetime of interest, in this case T2. The variable $v$ is a small offset we include in the fit in order to accommodate any noise in the signal such 
that the curve never reaches a true zero; it is not part of the theoretical description, but its existence is worth noting, as we use it in all of our fit functions. The amplitude at the half-life $T_{1 / 2}$ is $A / 2$ by definition, so substituting we get

$$
A / 2=A e^{\wedge}\left(-T_{1 / 2} / \tau\right)
$$

and thus the relationship between the lifetime and the half-life is

$$
\tau=T_{1 / 2} / L N(2) .
$$

Therefore, plugging in our 13.35 seconds for $\mathrm{T}_{1 / 2}$ gives us a $\mathrm{T} 2$ value of 19.26 seconds. We will use the relationship in equation 2 often. Even still, the most consistent way to pull a number for $\mathrm{T} 2$ is to fit the data curve by tweaking the values of $\mathrm{A}$ and $\mathrm{T} 2$ in a theoretical curve described by equation 1 until our theoretical curve matches the data curve as closely as possible. We can accomplish this using the fmincon function in $M A T L A B$, or any other tool that includes a solver or optimizer function. The red line through the blue data points in Figure 30 represents the theoretical fit solution, which returned a T2 value of 19.36 seconds. The two methods match to a tenth of a second, which is the highest precision we usually care about for lifetime measurements. We will most often use the fit approach because it allows for more consistent analysis and, if set up properly, yields results at the click of a button. From this point on, unless otherwise noted, data will be presented as blue dots and theoretical fits as red curves. 


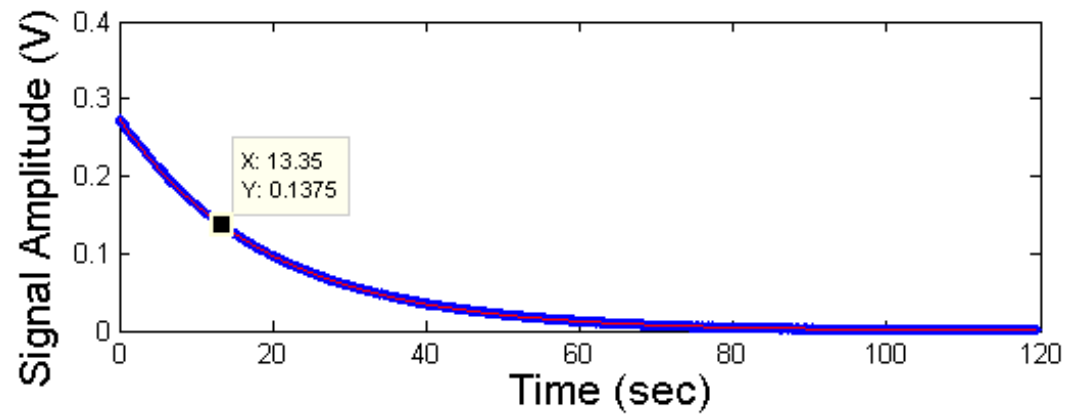

Figure 30. The FID profile with smaller windows highlights both the approximate half-life and the theoretical fit represented by the red curve through the data. Both approaches return a $\mathrm{T} 2$ value of about 19.3 seconds. For data expressed this way, the blue dots represent the measured data and the red curve is the theoretical fit.

Repeating the FID test 10 times over the course of about an hour, we determined the uncertainty in a set of T2 measurements (defined as the standard deviation of the statistical data set divided by the square root of one less than number of data points in the set) to be on the order of 10 milliseconds. Therefore, we consider our FID fit measurements to be quite repeatable and reliable.

So that's an example of our industry standard method for measuring T2 for ${ }^{129} \mathrm{Xe}$. Things tend to get more complicated when we deal with ${ }^{131} X e$, the nucleus of which is spin-3/2 as opposed to the spin-1/2 nucleus of ${ }^{129} \mathrm{Xe}$. The higher-order spin means that the nucleus can occupy more spin states (four to be exact: $-3 / 2,-1 / 2,+1 / 2$, and $+3 / 2$ ). The $-1 / 2$ and $+1 / 2$ states, which are the only options for ${ }^{129} \mathrm{Xe}$, make the atom a simple magnetic dipole. However, the ${ }^{131} \mathrm{Xe}$ atom also has an electric quadrupole in the higher-order spin states of $+/-3 / 2$. 


\section{a. The ${ }^{131}$ Xe Isotope and Electric Quadrupole Coupling}

For a nucleus such as that of the ${ }^{131} \mathrm{Xe}$ atom, we are no longer dealing with only spin-up and spin-down conditions, which refer to the $+1 / 2$ and $-1 / 2$ spin states of a spin-1/2 particle or atom; we now also have atoms in the $+3 / 2$ and $-3 / 2$ states, which can have different Larmor precession frequencies due to asymmetries in the electric field gradient across the cell wall. The reason this is so detrimental to us is that the precession frequencies of the atoms in the quadrupole states are slightly different from the dipole atoms, which can lead to dramatic changes in the shape of the decay curve. As with any waveform composed of multiple frequencies, beating appears in the signal, as shown in an extreme case in Figure 31 below.

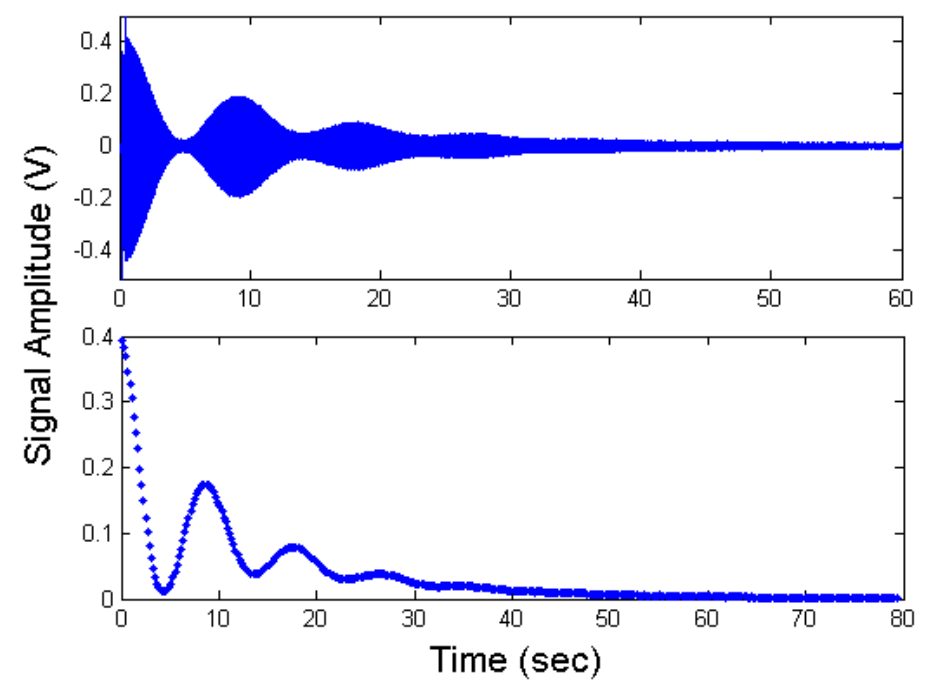

Figure 31. Example ${ }^{131} \mathrm{Xe}$ decay curve with strong electric quadrupole coupling. The raw signal (top) is processed in the same way as it was for ${ }^{129} \mathrm{Xe}$ above in order to generate the profile (bottom). 
Clearly this looks nothing like a simple decay curve, and there seems no way to figure out precisely where the signal reaches half of its maximum amplitude, which means we cannot very well determine $\mathrm{T} 2$ by using the half-life. But this is physics, and there is surely a way to theoretically describe the shape of the curve given what we know; presumably we have three separate frequencies - our center dipole frequency and one on each side of the center due to the two quadrupole-coupled spin states. If we perform an FFT on this data run, we see the frequency signature traced in blue in Figure 32 below.

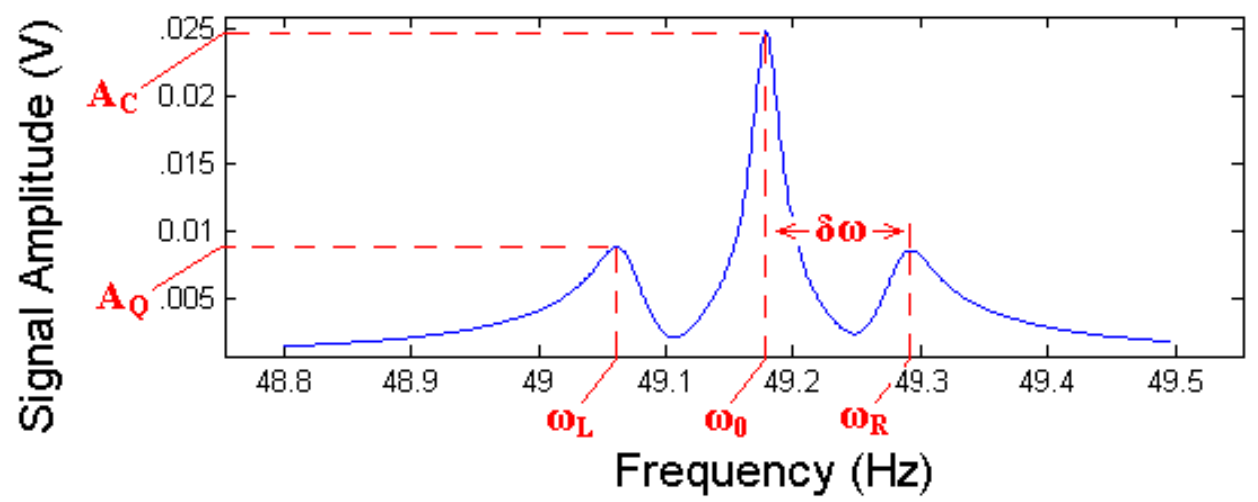

Figure 32. Frequency signature of the ${ }^{131}$ Xe decay curve above, with relevant fit parameters labeled.

Clearly, there are three distinct frequencies in this signal. The center frequency is typically the dominant frequency, which represents the precession of the ${ }^{131} \mathrm{Xe}$ atom group whose wave-function is in a superposition of the $+1 / 2$ and $-1 / 2$ spin states. The side frequencies represent the precession of the atom group whose wave-function is a superposition of the spin-3/2 and spin-1/2 states (+ and - pairs). The precession frequencies for all of these superposition states are subject to the magnitude of the electric field gradient the atoms experience while in contact with the cell walls. Asymmetries in electric field gradients across the cell cause the differences in 
frequencies, and the cell walls are where such asymmetries tend to exist (albeit extremely small asymmetries).$^{7,8}$

So now we need to fit the profile as we did with ${ }^{129} \mathrm{Xe}$, but first we must derive the theoretical equation that we hope will describe the profile. The raw data after the pulse should be described by the superposition of the three decaying sine waves

$$
y=A_{C} \sin \left(\omega_{C} t\right) e^{\wedge}\left(-t / T 2_{C}\right)+A_{Q}\left[\sin \left(\omega_{L} t\right)+\sin \left(\omega_{R} t\right)\right] e^{\wedge}\left(-t / T 2_{Q}\right),
$$

where $\mathrm{A}_{\mathrm{C}}$ and $\mathrm{A}_{\mathrm{Q}}$ are the initial transverse amplitudes of the center and quadrupole decays, respectively; $\mathrm{T} 2_{\mathrm{C}}$ and $\mathrm{T} 22_{\mathrm{Q}}$ are the respective transverse lifetimes. We are assuming here that the two quadrupole states are equally populated and have a common lifetime, and therefore we use the same $\mathrm{A}_{\mathrm{Q}}$ and $\mathrm{T} 2_{\mathrm{Q}}$ value for both. If we assume farther that the precession frequency separation $\delta \omega$ is equal for the two quadrupole states, we can write the quadrupole frequencies as

$$
\begin{aligned}
& \omega_{L}=\omega_{C}-\delta \omega, \\
& \omega_{R}=\omega_{C}+\delta \omega
\end{aligned}
$$

and substitute them into equation 3 , which after some trigonometric reduction becomes

$$
y=\sin \left(\omega_{C} t\right)\left[A_{C} e^{\wedge}\left(-t / T 2_{C}\right)+2 A_{Q} \cos (\delta \omega t) e^{\wedge}\left(-t / T 2_{Q}\right)\right] .
$$


So we have now successfully isolated the center-frequency sine wave, and we can directly see the quadrupole-frequency cosine term that causes the beating in the signal. To obtain the profile, we want to use only the maximum and minimum values of each cycle of the center-frequency sine wave, or in other words set the magnitude of the sine wave equal to one at all times. Also, it is possible for the combined amplitudes from the quadrupole atoms to outweigh the center amplitude, which would theoretically produce negative values in the profile. Of course, we can only measure a positive signal as described by the FFT-based profile, so we take the absolute value of the curve and ultimately acquire our theoretical description of the ${ }^{131} \mathrm{Xe}$ FID profile:

$$
y=\left|A_{C} e^{\wedge}\left(-t / T 2_{C}\right)+2 A_{Q} \cos (\delta \omega t) e^{\wedge}\left(-t / T 2_{Q}\right)\right|+v .
$$

Using this as our fit function, as we did with equation 1 for ${ }^{129} \mathrm{Xe}$, we find the solution illustrated in Figure 33 below by using the values listed in Table 1. Note that, even though equation 4 calls for the frequency separation in radians, it is given in $\mathrm{Hz}$ below for ease of comparison with the FFT image in Figure 32 above.

Table 1. Values used to fit ${ }^{131} \mathrm{Xe}$ decay curve shown in Figure 33 below.

\begin{tabular}{|c|c|c|}
\hline Variable & Value & Unit \\
\hline $\boldsymbol{A}_{\boldsymbol{C}}$ & 0.1792 & Volts \\
\hline $\boldsymbol{T} \boldsymbol{2}_{\boldsymbol{C}}$ & 15.8562 & Seconds \\
\hline $\boldsymbol{A}_{\boldsymbol{Q}}$ & 0.11065 & Volts \\
\hline $\boldsymbol{T} \boldsymbol{2}_{\boldsymbol{Q}}$ & 7.7817 & Seconds \\
\hline $\boldsymbol{\delta} \boldsymbol{f}$ & 0.11034 & Hertz \\
\hline
\end{tabular}




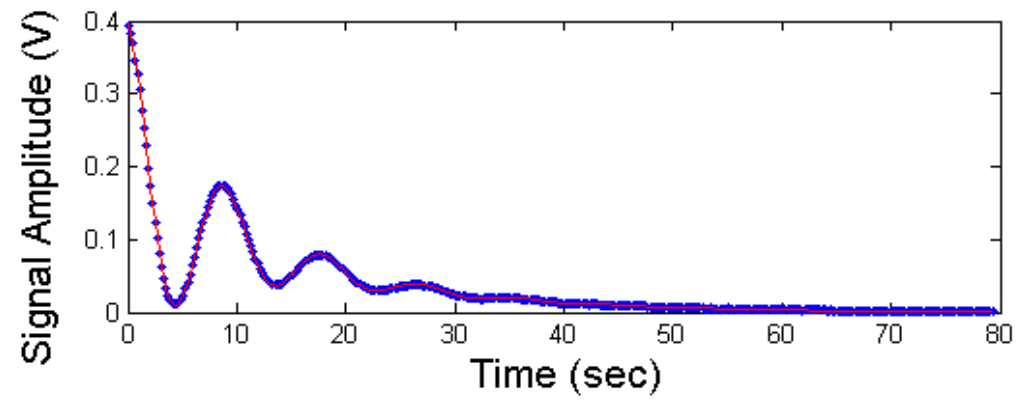

Figure 33. Quadrupole-coupled ${ }^{131}$ Xe decay curve (blue) with fit solution overlaid (red).

We can check the validity of this solution by measuring the frequency separation visually and comparing it to the fit value. Close inspection of Figure 23 yields an average separation of about $0.11565 \mathrm{~Hz}$. This is within $5 \%$ of the fit value, which suggests that the solution is valid to the resolution required. The relative amplitudes $A_{Q}$ and $A_{C}$ could provide even further validation, but the comparison between fit values and FFT is more difficult to draw in this case because the signal is generated by the total population of atoms, which relates not just to the vertical amplitude of the FFT but to the entire area under the FFT curve, making it more trouble than it is worth as an unnecessary detail.

One of the most common problems that arise when fitting these quadrupole-coupled decay curves emerges when we do not use the correct start time (i.e., immediately following the pulse). With a pure exponential decay curve such as with ${ }^{129} \mathrm{Xe}$, the start time should not matter since the curve follows the same pattern throughout the test. However, when dealing with multiple frequencies, the passage of time following the pulse leads to greater phase misalignment between the spins of the atoms of different frequencies. Since our fit function does not allow for an initial phase offset between the different atomic populations, if we do not select the correct start time, the optimizer cannot find a very good solution. This problem is illustrated in Table 2 and Figure 34 below, 
using the exact same data set as above for comparison, but with the start time shifted one full second forward.

Table 2. Values used to fit ${ }^{131}$ Xe decay curve shown in Figure 34 below.

\begin{tabular}{|c|c|c|}
\hline Variable & Value & Unit \\
\hline $\boldsymbol{A}_{\boldsymbol{C}}$ & 0.13963 & Volts \\
\hline $\boldsymbol{T} \boldsymbol{2}_{\boldsymbol{C}}$ & 21.6583 & Seconds \\
\hline $\boldsymbol{A}_{\boldsymbol{Q}}$ & 0.07707 & Volts \\
\hline $\boldsymbol{T} \boldsymbol{2}_{\boldsymbol{Q}}$ & 8.3459 & Seconds \\
\hline $\boldsymbol{\delta} \boldsymbol{f}$ & 0.1242 & Hertz \\
\hline
\end{tabular}

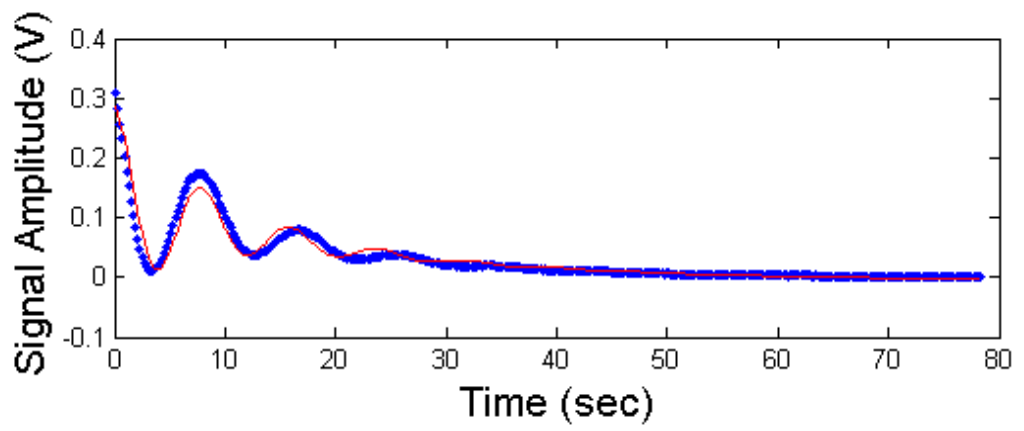

Figure 34. Quadrupole-coupled ${ }^{131} \mathrm{Xe}$ decay curve (blue) and fit (red), taking the start time to be one full second after the pulse finishes.

Clearly, the fit does not match the data profile nearly as well. The real problem is the reported value for $\mathrm{T} 2_{\mathrm{C}}$, which is almost $37 \%$ high. For only a one-second difference this is a huge discrepancy in results, which highlights the importance of pinpointing the start time accurately. In turn, this provides us with more incentive to use the $\pi / 2$-puse for this method (as opposed to using a small drive and then pulling it) simply because it is much easier to visually determine the start of the run with a pulse. 
It is also meaningful to point out that the quadrupole effects are not always so pronounced. A more typical example of a ${ }^{131} \mathrm{Xe}$ decay curve is presented in Table 3 and Figure 35 below.

Table 3. Values used to fit ${ }^{131}$ Xe decay curve shown in Figure 35 below.

\begin{tabular}{|c|c|c|}
\hline Variable & Value & Unit \\
\hline $\boldsymbol{A}_{\boldsymbol{C}}$ & 0.15497 & Volts \\
\hline $\boldsymbol{T}_{\boldsymbol{C}}$ & 15.4805 & Seconds \\
\hline $\boldsymbol{A}_{\boldsymbol{Q}}$ & 0.078066 & Volts \\
\hline $\boldsymbol{T}_{\boldsymbol{Q}}$ & 11.8101 & Seconds \\
\hline $\boldsymbol{\delta} \boldsymbol{f}$ & 0.024487 & Hertz \\
\hline
\end{tabular}

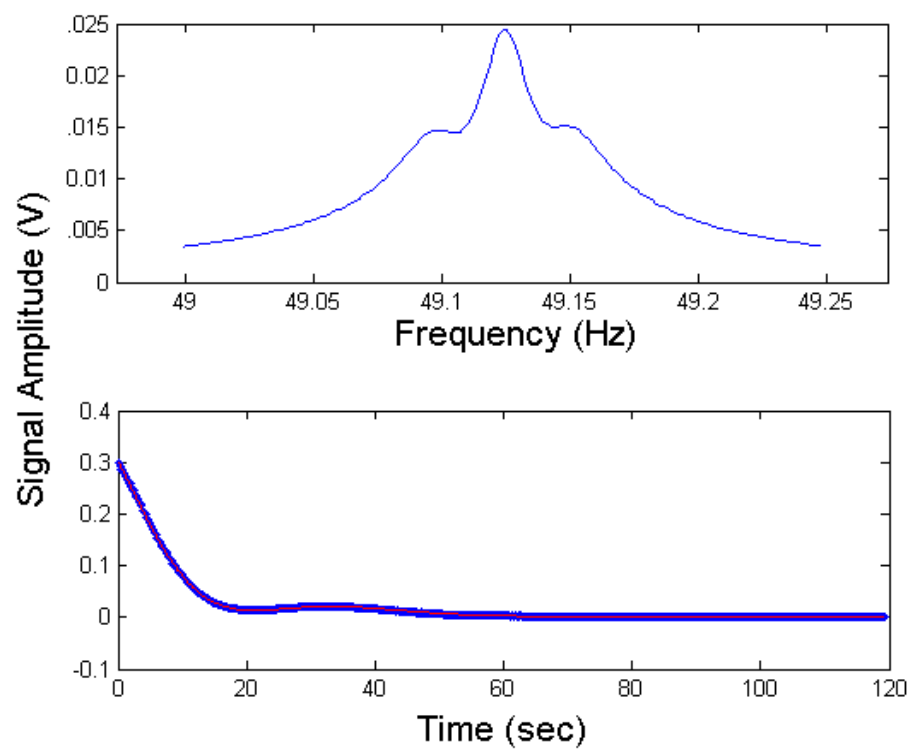

Figure 35. Example ${ }^{131} \mathrm{Xe}$ decay with more typical quadrupole effects.

This is a real problem when we deal with cells that have noticeable but very small quadrupole coupling. If the effects are negligibly small, we can get away with fitting the curve using equation 1 , simply ignoring the quadrupole altogether. However, when the effects are large enough to alter the shape of the curve but small enough that we cannot determine the frequency separation just from looking at the FFT, the optimizer usually 
has a difficult time finding the right solution. In such cases, we can still get a reasonable estimate of the T2 by using equation 1 and accepting that it is not a perfect fit.

Another persistent issue stems from the fact that the quadrupole peaks are never perfectly symmetric about the center. In fact, the quadrupole coupling theoretically shifts the center frequency slightly due to higher-order effects, and thus inherently $\delta \omega$ is never exactly identical on both sides nor are the relative peak amplitudes. This can cause incurable discrepancies between our fit function and the actual data, but such discrepancies have never proven to be significant enough to worry about.

Still, in the event that we would want to really delve into these issues, the solution would be to fit the full sine wave of the data set rather than the simplified profile, and include in our fit function all three atomic groups with room for individual amplitudes, frequencies, and even initial phase offsets. Such an operation would add quite a bit of strain to the optimizer, partly because of the increased number of solvable parameters but mainly due to the incredibly large data set it would be working with. It is a good option to have, but for the time being our level of accuracy is sufficient.

\section{b. Free Induction Decay by Pulling a Small Sustained Drive}

As previously mentioned, we could also have done this test using the drive from the function generator and disconnecting it at the start of the data collection. The main reason 
we prefer the pulse is that it is consistent both in magnitude and the amount of time it takes, so determining the exact time that the actual data run begins is usually easier with the pulse. Still, to confirm our assumptions we repeat the test using first the function generator and then the pulse, the results of which are shown in Figure 36 below. The difference in amplitude between the two drive methods stems from the fact that the small sustained drive stimulates the atomic precession much more slowly, so atoms lose their spin states while still in the process of stimulation. With the pulse, however, almost all of the polarized xenon atoms reach full transverse stimulation very quickly.
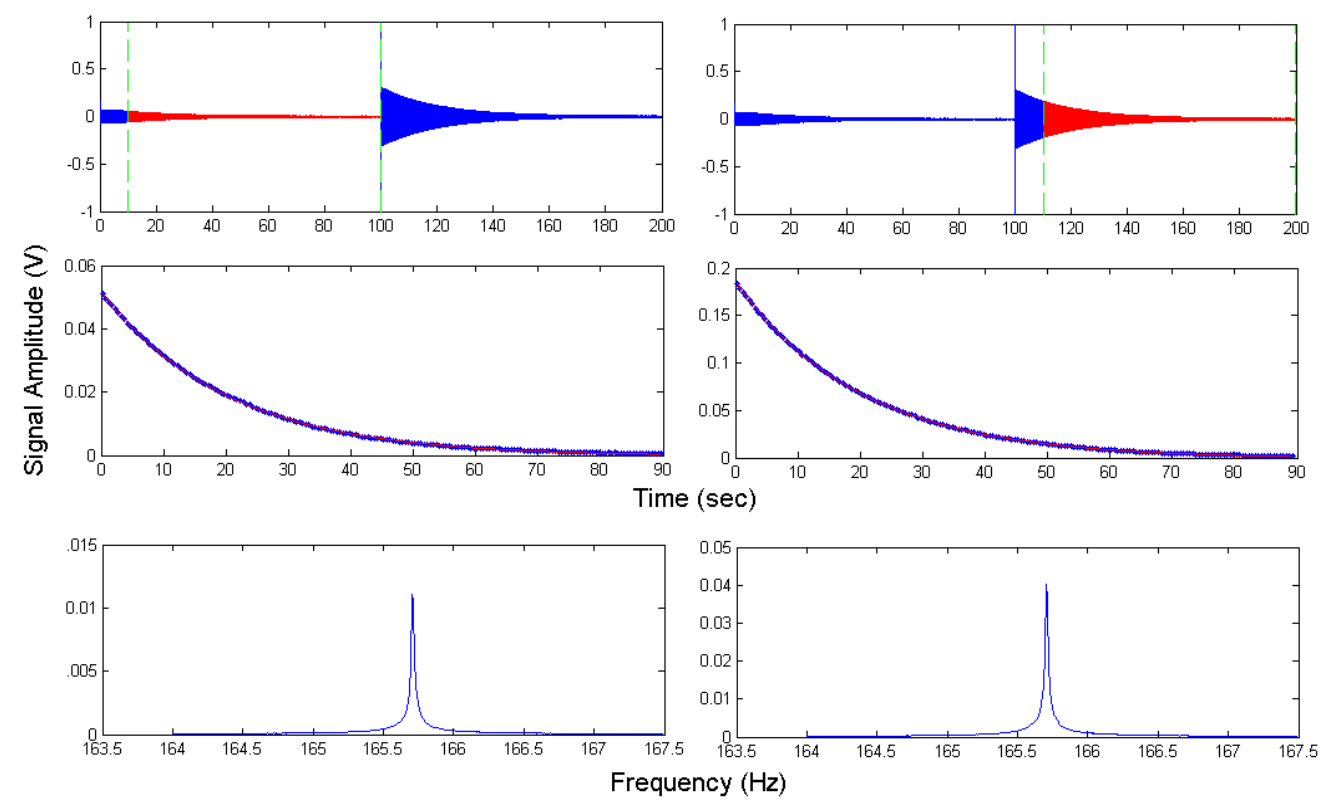

Figure 36. Results for ${ }^{129}$ Xe FID performed twice: first by removing a small sustained drive (highlighted on the left), second by $\pi / 2$ pulse (right). For data expressed this way, the red portion of the raw signal indicates the portion of the data from which the fitted profile was constructed. The vertical green dashed lines indicate the portion of the data from which the FFT was constructed to find the center frequency. 
Based on the fit solutions, the FID from the pulled sustained drive yielded a T2 of 20.032 seconds while that from the pulse gave 20.084 seconds: a difference of only $0.26 \%$. As expected, the two methods are essentially identical except for the significantly higher signal when using the pulse. However, this is apparently not the case when dealing with the electric-quarupole-coupled ${ }^{131} \mathrm{Xe}$ isotope, as presented in Table 4 and Figure 37 below.

Table 4. Values used to fit ${ }^{131}$ Xe decay curve shown in Figure 37 below.

\begin{tabular}{|c|c|c|c|}
\hline Variable & Drive Data Value & Pulse Data Value & Unit \\
\hline $\boldsymbol{A}_{\boldsymbol{C}}$ & 0.02981 & 0.1878 & Volts \\
\hline $\boldsymbol{T}_{\boldsymbol{C}}$ & 23.0116 & 15.7525 & Seconds \\
\hline $\boldsymbol{A}_{\boldsymbol{Q}}$ & 0.0213 & 0.096213 & Volts \\
\hline $\boldsymbol{T} \boldsymbol{2}_{\boldsymbol{Q}}$ & 8.5242 & 12.3098 & Seconds \\
\hline $\boldsymbol{\delta} \boldsymbol{f}$ & 0.039463 & 0.028556 & Hertz \\
\hline
\end{tabular}
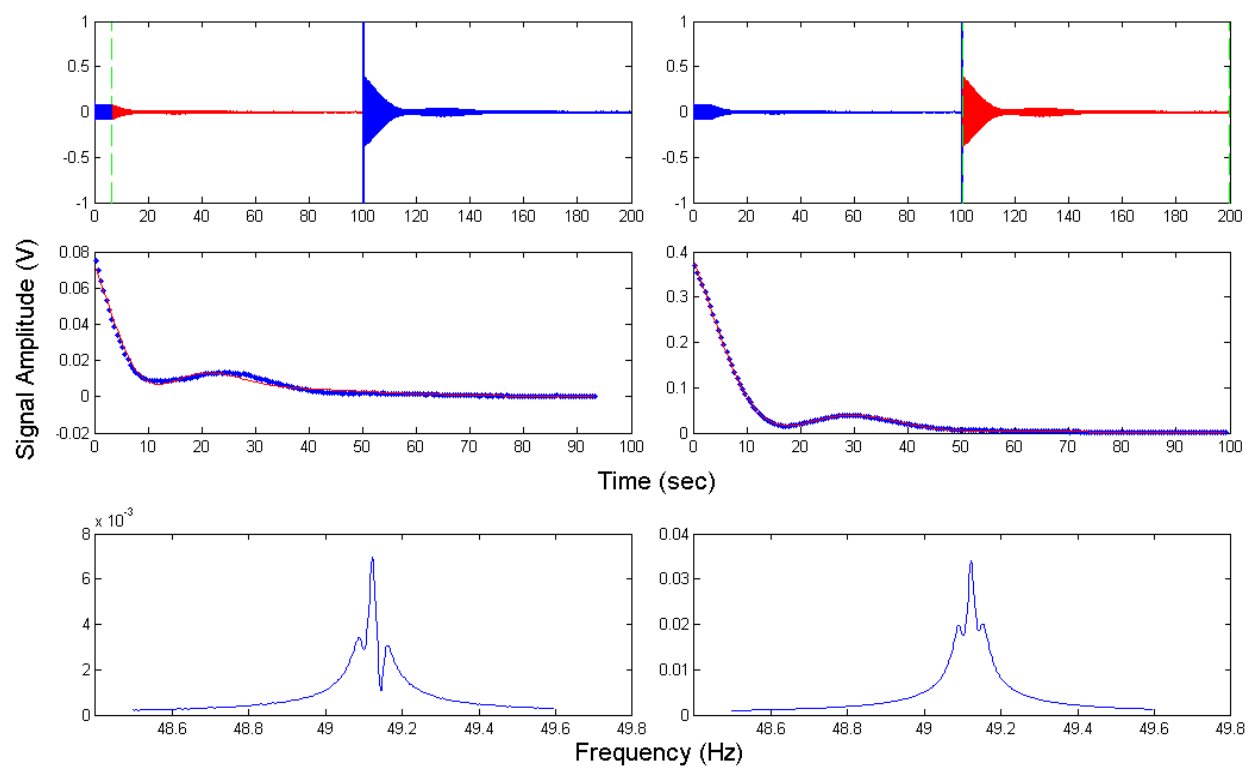

Figure 37. Raw data from FID of ${ }^{131}$ Xe performed both by pulling the sustained drive (left) and by pulse (right). Unlike with ${ }^{129} \mathrm{Xe}$, there is a large discrepancy between the two stimulation methods. 
Visually, we can immediately see a difference between the two curves, and clearly the fit does not match well for the decay using the pulled sustained drive. Taking the pulse data values to be correct (based on the clean fit), the sustained drive run gives us a reported $\mathrm{T} 2 \mathrm{C}$ that is $46.08 \%$ too high.

The FFT (bottom) offers some insight in that the center peak is shifted slightly to the left for the sustained drive test. This suggests that the sustained drive, which must have been at a slightly lower frequency than the natural Larmor frequency, had some residual effect on the ${ }^{131} \mathrm{Xe}$ population even after being disconnected. There should be no such residue; as soon as the drive is removed the atoms should have no recollection of it and should go on behaving in their natural manner. To investigate this further, we repeat the test using a sustained drive with off-resonance frequency (something like $0.2 \mathrm{~Hz}$ high), the result of which is shown in Figure 38 below. Note that the pulse was also set to the same offresonance frequency for this run. 

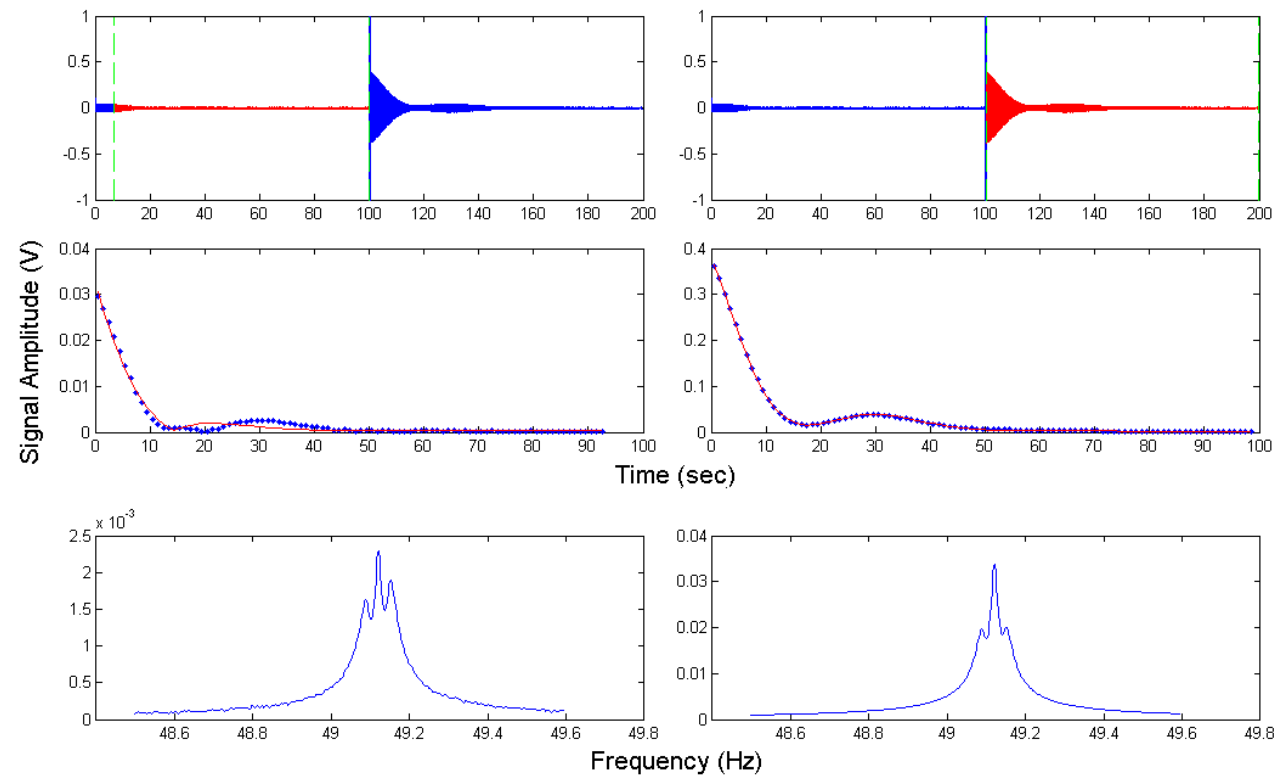

Figure 38. Repeat of ${ }^{131}$ Xe FID, this time using a drive frequency around $49.3 \mathrm{~Hz}$ (intentionally above resonance). Again, the small sustained drive was pulled (left) and then a pulse was used (right).

Going by the fit, the off-resonance pulse (shown on the right) made no difference, and quantitatively it yielded nearly identical results to the on-resonance test (within 0.1 seconds for both the center and quadrupole-induced T2 times). However, the pulled drive run again displays different behavior, and due to the large asymmetry in the FFT, the fit function fails as discussed earlier. There seems to be no residual population at the drive frequency (around $49.3 \mathrm{~Hz}$ ), which is expected and encouraging, but clearly the quadrupole-coupled side peaks have been effected in a way that remains after the drive is disconnected. Perhaps coincidentally, our drive frequency was higher than the natural resonance, and the higher-frequency side peak has been exaggerated. As a final query on this matter, we try the same test once more, this time using a drive frequency below the natural Larmor frequency, shown in Figure 39, to see if the FFT asymmetry flips. 

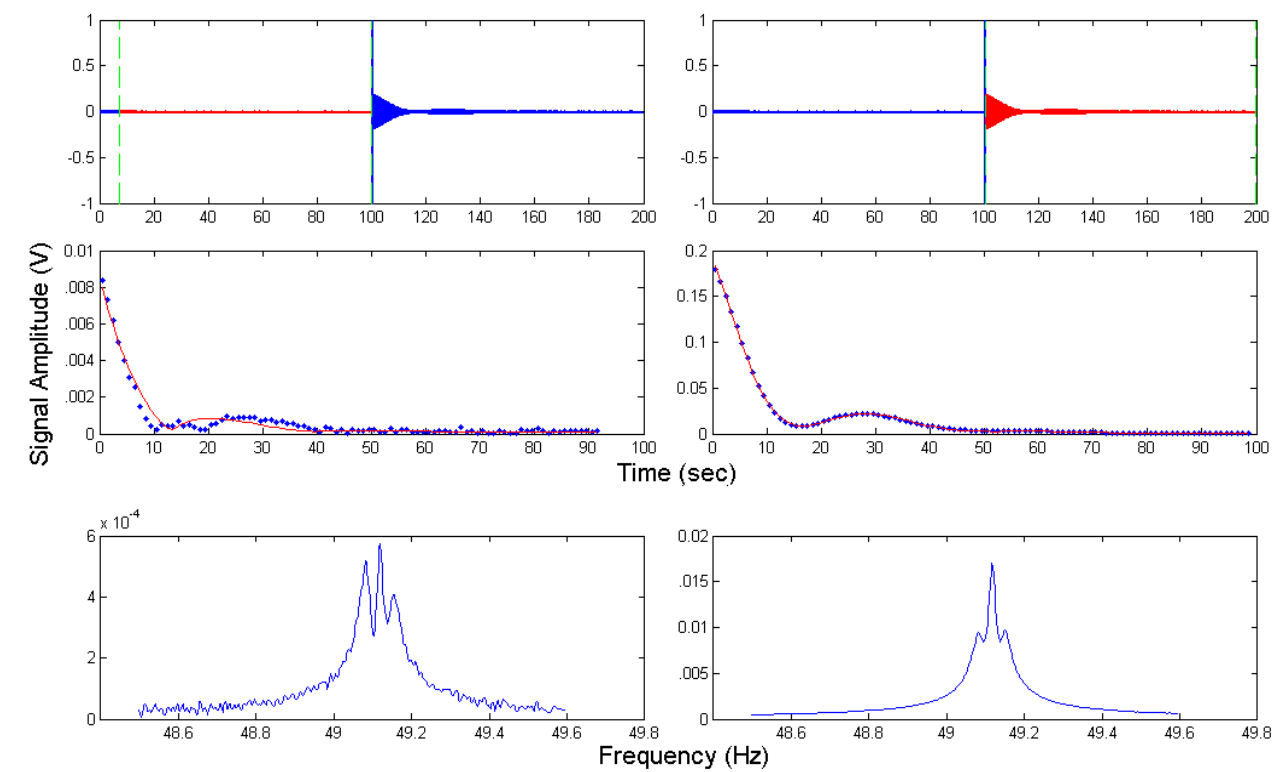

Figure 39. Repeat of ${ }^{131}$ Xe FID, this time using a drive frequency around $48.95 \mathrm{~Hz}$ (intentionally below resonance). Again, the small sustained drive was pulled (left) and then a pulse was used (right).

Indeed, the asymmetry of the peaks depends on the drive frequency relative to the natural resonance frequency. The explanation most likely comes down to the bandwidth of the drive. See, the small sustained drive is present for a long time before it is pulled (in order to bring the atoms to steady state), giving it a much narrower bandwidth than the pulse, whose duration is only about 0.2 seconds. The group of wall-adsorbed atoms whose quadrupole-induced natural frequency is closer to the drive frequency clearly gets more effectively stimulated by the narrow-band sustained drive, while the wide-band pulse performs much more uniform stimulation across the entire ${ }^{131} \mathrm{Xe}$ population.

Upon very close inspection of the pulsed data sets, the right peak is $.0003 \mathrm{~V}$ higher in amplitude than the left peak when driving to the right of resonance, and $.0002 \mathrm{~V}$ higher than the left peak when driving to the left of resonance, so there is a measureable 
difference. We can thus convince ourselves that drive bandwidth is in fact the source of the discrepancy between the two drive methods.

We conclude that the pulse drive works better for the FID T2 tests because it provides ease and consistency in determining start times, higher signal amplitude, and much more uniform stimulation of ${ }^{131} \mathrm{Xe}$ which allows our simplified fit function to do the job well. So that covers our industry standard method for measuring $\mathrm{T} 2$, but theoretically there is another way, and although we rarely actually use it, there are important principles we can discuss while introducing what we call the T2 Growth Method. We will get to that shortly, but first let us take a look at our industry standard technique for measuring T1: the Delayed Pulse Method.

\section{Measuring T1 Using the Delayed Pulse Method}

As discussed earlier, $\mathrm{T} 1$ is the lifetime of real importance to us during cell testing because the value of $\mathrm{T} 1$ is the upper limit for $\mathrm{T} 2$ for a given cell at a given temperature. The decay of the longitudinal spin alignment comes from collisions with either the cell walls or the other gas atoms in the cell which can destroy the spin states of the xenon atoms. The difference now is that those collisions should be the only contributing factors to the decay, as opposed to the transverse spin decay which also depends on magnetic field nonuniformities in the cell. As such, T1 must be longer than T2, but we still expect the T1 decay curve to follow equation 1 in some form. 
However, the question still remains: how do we measure the longitudinal lifetime if our signal decays with the shorter transverse lifetime? Well, take the system with no transverse drive, where the xenon atoms are polarized along the positive $z$ axis but there is no precession. If we use a $\pi$ pulse, which flips the xenon spins $180^{\circ}$, we know that the amount of time it takes for only 1/e of the xenon atoms to remain in the flipped orientation should be T1. Even still, we cannot monitor this transition of the $z$ polarization because there is no common transverse component precession, so there is no signal.

However, if we stimulate the precession using a $\pi / 2$ pulse at some time during this transition, then the phase of the precession for the atoms that remain negatively polarized at the time of the pulse will be shifted $180^{\circ}$ from those that have regained positive polarization. In other words, the two oppositely polarized atomic populations cause destructive interference which cancels out a portion of the signal following the pulse. Figure 40 illustrates this concept.

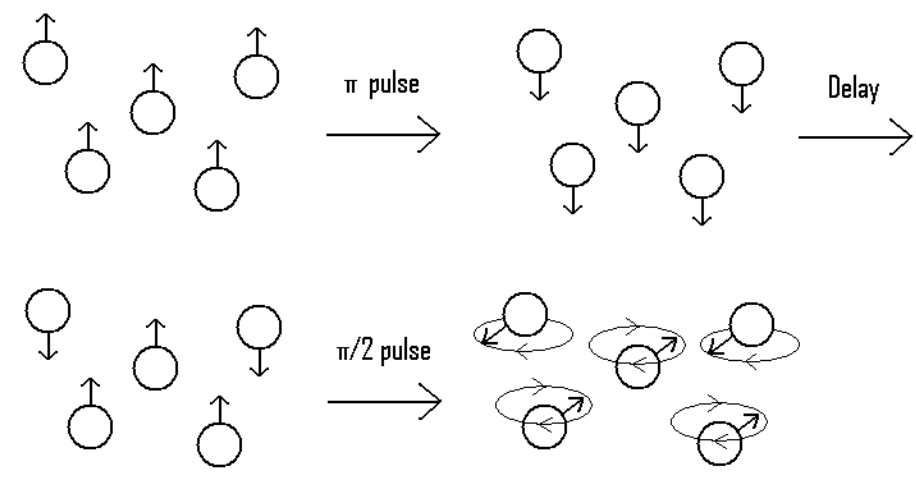

Figure 40. Following a $\pi$ pulse, a $\pi / 2$ pulse puts xenon atoms of opposing polarizations into precession 180 degrees out of phase from each other, resulting in destructive interference. 
So, we begin the test with a $\pi$ pulse and allow some time delay before applying the $\pi / 2$ pulse. If we vary our delay time for different tests and record the amplitude of the signal immediately following the $\pi / 2$ pulse for each, we will begin to see the longitudinal spin decay curve. With short delay times, the signal is generated by the dominant population in the negative polarization state. As the delay increases, the signal reaches a zero which should represent the half-life, both polarization states being equally populated at the time of the $\pi / 2$ pulse. If the delay is extended past the half-life, the signal grows back up as the population of positively polarized xenon atoms begins to outweigh the opposite spin state once again. Figure 41 shows a few sample data runs with varying delay times, as well as the overall curve after recording the amplitudes of all the data runs.
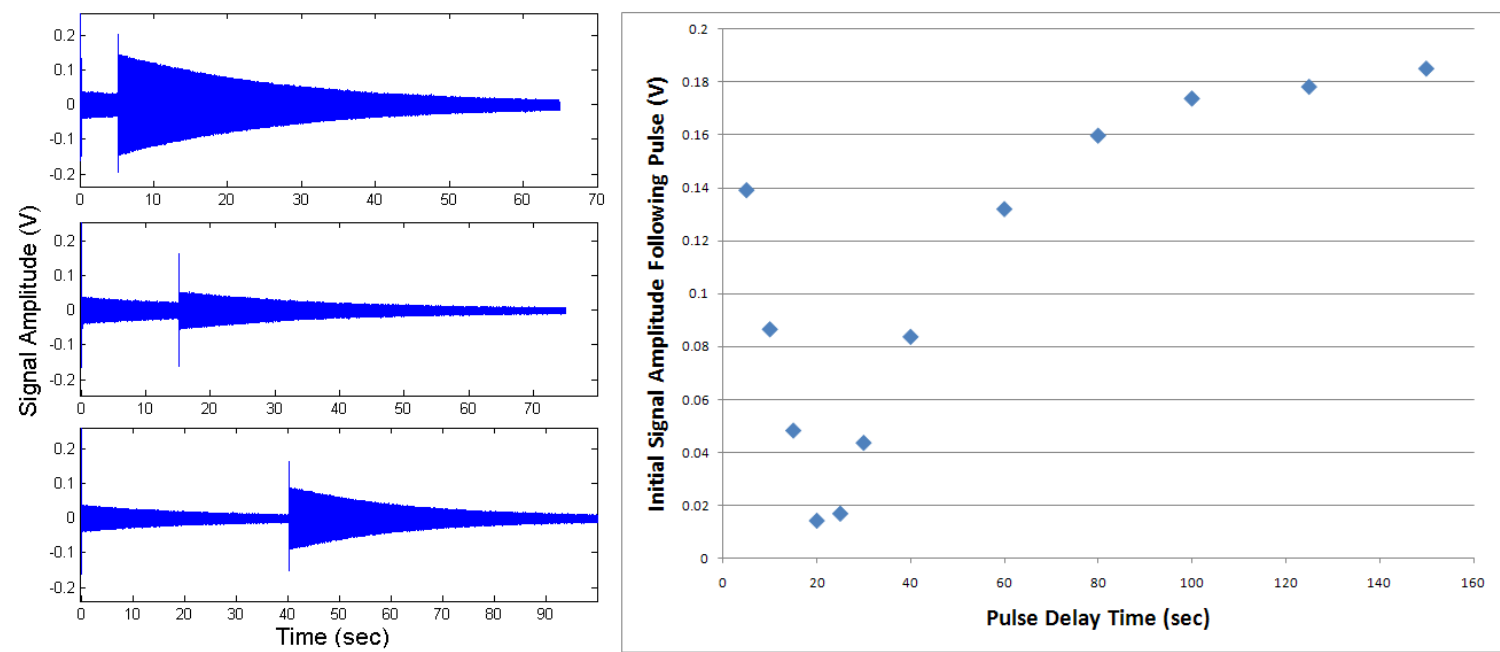

Figure 41. Sample data runs for the delayed pulse method (left) and the full curve from recording the initial amplitude after the pulse for each of the data runs performed (right).

Again, we have our two ways to derive the lifetime. The half-life corresponds to the zero crossing, which visually seems to occur at about 22 seconds, from which equation 2 gives 
us a T1 of 31.74 seconds. We can also fit the data; we just need to modify it a bit first. See, the reason the signal amplitude grows back up is simply because we cannot measure negative amplitudes, but conceptually the sign of the signal on either side of the half-life should be opposite if we think of the curve as representing the population of a particular polarization state over time. In this light, we can multiply the amplitudes of all the points after the half-life by -1 .

Now we have something that much more closely resembles our exponential decay curve described by equation 1, as shown in Figure 42 (left). We can fit this with equation 1 (noting that the vertical offset $v$ will be much larger than usual), and we get a T1 value of 30.69492 seconds. We can also choose to make the points before the half-life negative, effectively tracking the population of the other polarization state. The curve in that case should be described by subtracting the signal generated by the decaying population at any time from the signal that would occur if all the atoms were in the final state. More simply put, we subtract the decay curve from the steady state amplitude, giving us

$$
y=A\left[1-e^{\wedge}(-t / \tau)\right]+v
$$

which we will refer to as our growth equation. In this case $\tau$ represents T1. The result of this modification to the data is also shown in Figure 42 (right), and the fit using equation 5 yields a T1 of 30.69495 seconds. 

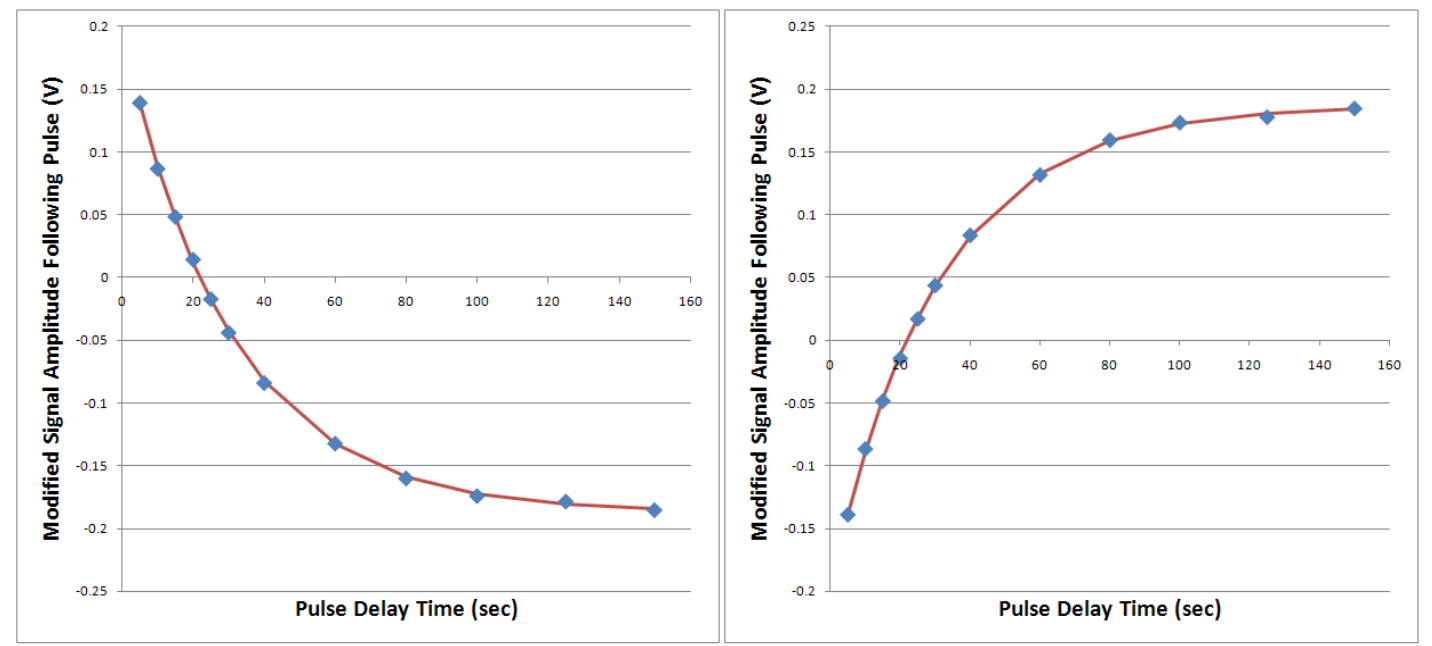

Figure 42. The shape of the delayed pulse curve has been modified to represent the populations of the particular spin groups; the left curve represents the decay of the spin-down group and the right curve the growth of the spin-up group following the $\pi$ pulse; both curves are shifted down due to the vertical offset inherent in measuring the absolute value.

The reason there is a discrepancy between the two solutions (albeit very small) is that there is noise in the signal, so the raw data never reaches a true zero, and that offset gets carried over when we flip the data points. In this particular example we would not worry at all about such a small noise level, but when dealing with lower signals the discrepancy can be significant. Either way, the fit results are much more reliable than our visual estimate of the half-life, and in this case it is sufficient to say the T1 is around 30.7 seconds.

Now, it is nice to see the data this way because it gives us a curve we are familiar with, but the fastest and most consistent way to pull results is to leave the data alone and instead modify our fit function. We know we essentially have a decay curve, and presumably the initial amplitude when all of the xenon atoms are spin-down should 
match the final amplitude when they have all returned to the spin-up state. For a decay curve, however, we are used to seeing some initial amplitude and a final value of zero. So what we really want to use to describe the delayed pulse curve is

$$
y=\left|A e^{\wedge}(-t / \tau)-V\right|+v
$$

where $V$ is the offset (something close to $A / 2$ ) that allows the initial and final magnitudes of the curve to be equal. We take the absolute value so that the curve is always positive and thus will match the data, and $v$ is our usual offset variable to account for any noise such that the data would never cleanly reach exactly zero. The results of this fit function are shown in Figure 43 below.

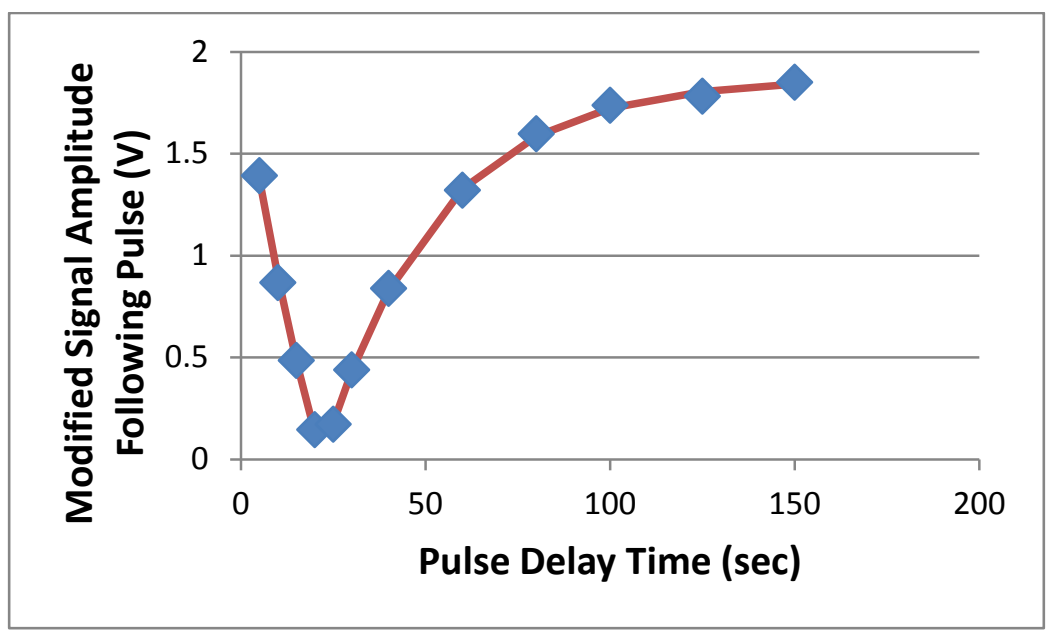

Figure 43. Fitted longitudinal decay curve obtained using the delayed pulse method. For the purpose of demonstrating the shape of the curve, this example contains more data points than are typically collected. 
Using equation 6 , we never have to touch the raw data, and we come out with an estimate for the longitudinal lifetime from the fit, which we will call $\mathrm{T} 1_{\text {fit }}$. On top of that, we can calculate the apparent half-life based on the fit values by setting $y=v$ (ideally zero) and solving for $t$ (which at that point is $T_{1 / 2}$ ):

$$
T_{1 / 2}=\left|T 1_{f i t} * L N(V / A)\right|
$$

and then we can solve for T1 from that value as well using equation 2, giving us another estimate of the lifetime based on the apparent zero crossing. We will call this value $\mathrm{T} 1_{0}$. So now we have very consistent means of determining T1 from both perspectives, and the solution values for this example are given in Table 5 below. What's more, we can get a reasonably precise approximation for the half-life with as little as four data points, saving a great deal of time.

Table 5. Delayed pulse solution values for curve in Figure 43 using equations 6, 7, and 2

\begin{tabular}{|c|c|}
\hline $\mathbf{A}$ & 3.804228 \\
\hline $\mathbf{T}_{\text {fit }}$ & 30.81729 \\
\hline $\mathbf{T 1}_{\mathbf{0}}$ & 31.55821 \\
\hline $\mathbf{V}$ & 1.863479 \\
\hline $\mathbf{V}$ & 0.007199 \\
\hline
\end{tabular}

Although intuitively it seems that results from a curve fit would be the most reliable, there are several reasons we prefer the value derived from the half-life. In order for a fit to be reliable we must have sufficiently many data points to define the shape of the curve, and since each data point can take up to ten minutes to generate (depending upon the actual value of T1) we rarely collect as many data points as in the previous example. We 
usually strive to get two data points on either side of the half-life, as shown in Figure 44 below. Of course, there is some guessing that goes on at first, but the slope defined by the first two data points gives us a rough idea of the half-life and helps us determine what delay times to try next.

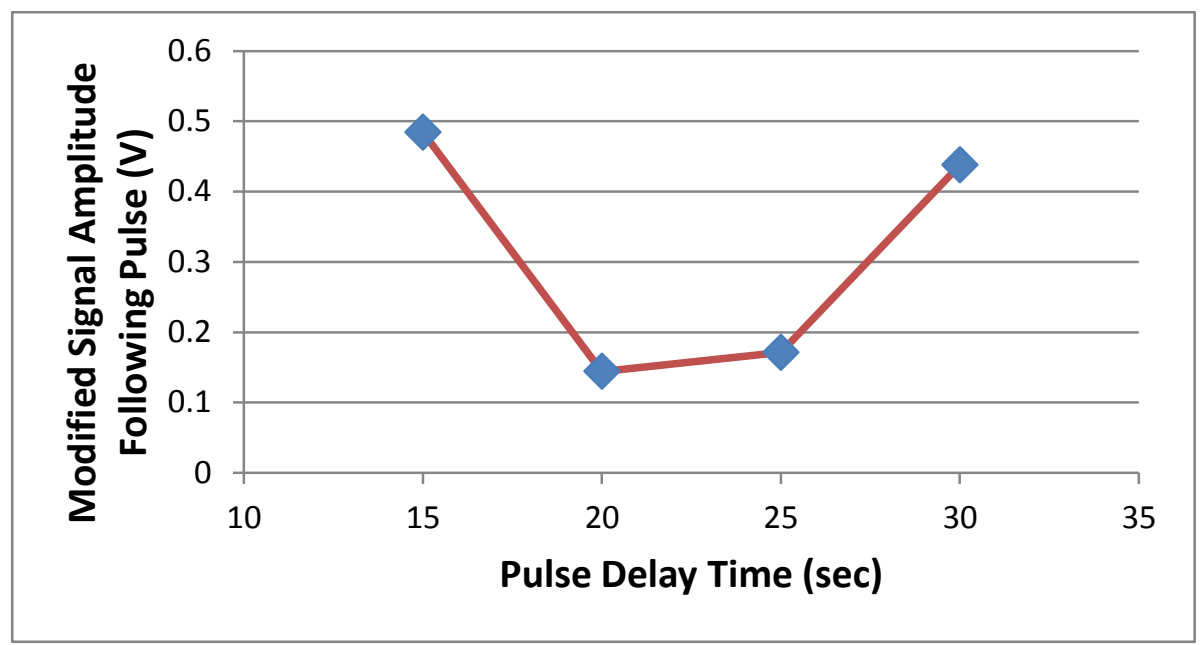

Figure 44. Typical results for the T1 delayed pulse method with only two data points on either side of the half-life. Although the shape of the decay curve cannot be resolved, a fairly accurate estimate of the half-life can be inferred from the apparent zero crossing.

Clearly, with so few points we cannot hope to resolve the shape of the curve, and so the fit-based $\mathrm{T} 1_{\text {fit }}$ is hardly ever reliable; the half-life-projected $\mathrm{T} 1_{0}$ value, on the other hand, cannot be very far off since the zero crossing must fall between the two minimum data points, and equation 7 yields relatively consistent estimates for the half-life even with such sparse data. Figure 45 below illustrates the outcomes from fitting this example longitudinal decay curve, varying the number of data points after the half-life that we use in the fit. 


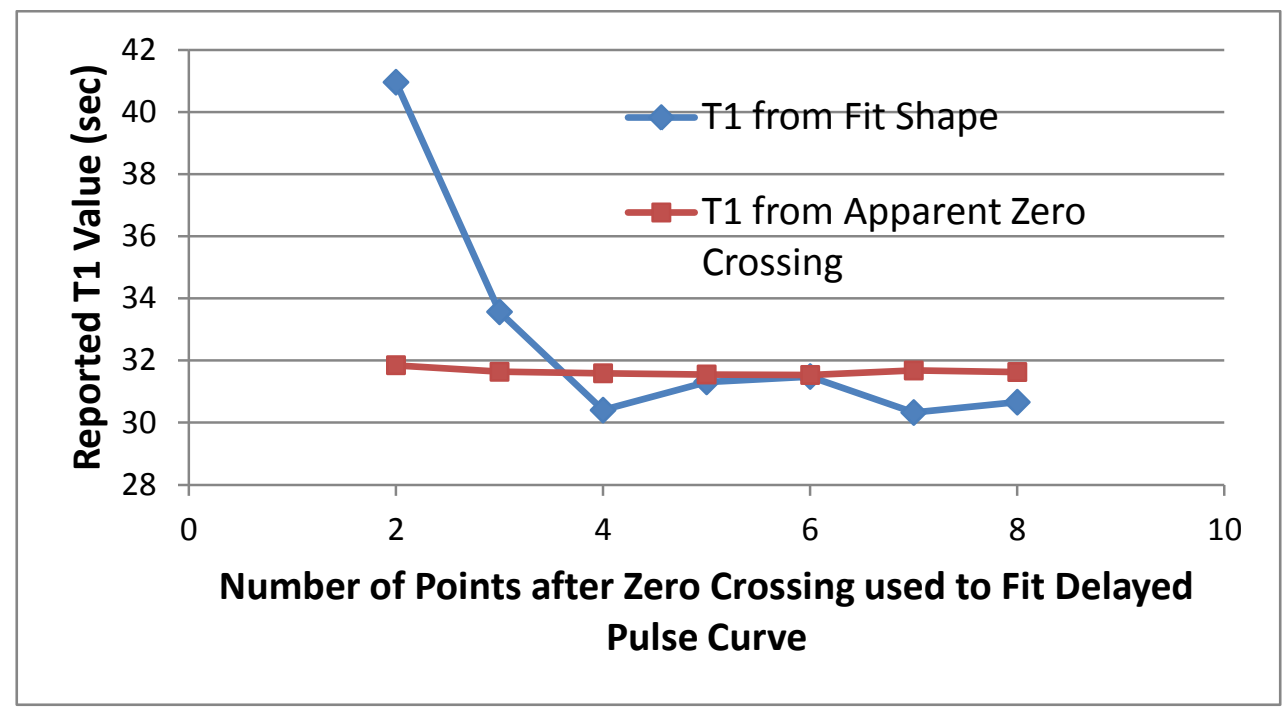

Figure 45. The $\mathrm{T1}_{0}$ value remains quite consistent regardless of how many data points are used in the fit, whereas the $\mathbf{T} 1_{\text {fit }}$ value is only reliable when many points are used.

Had we performed this test the way we usually do, using only four or so data points (two or three before the zero crossing and two or three after), the fit-based $\mathrm{T} 1_{\text {fit }}$ could have been as much as $33 \%$ off from the more accurate value we get by fitting the whole curve. Not to mention, the points closest to zero are inherently the noisiest, so the shape in that region will most likely always be distorted. On the other hand, we get highly consistent results by using equation 7 to find the half-life from the fit values and then putting that through equation 2 to find $\mathrm{T} 1_{0}$, regardless of the number of data points used. This, more than anything, is our motivation for relying on the half-life as our main point of analysis when performing the delayed pulse method.

Now, as with the free induction decay method for measuring T2, we must be a little more careful when measuring the longitudinal lifetime for ${ }^{131} \mathrm{Xe}$. Of course, we can try the same approach in analyzing delayed pulse data for the electric quadrupole-coupled 
isotope, and if nothing else we can usually obtain a clean enough curve to see the zero crossing. The issue is that at low signal amplitudes, as for the data points near the zero crossing, the asymmetries in the quadropule-coupled populations become more apparent, as shown in Figure 46 below, and therefore our fit function becomes less valid.

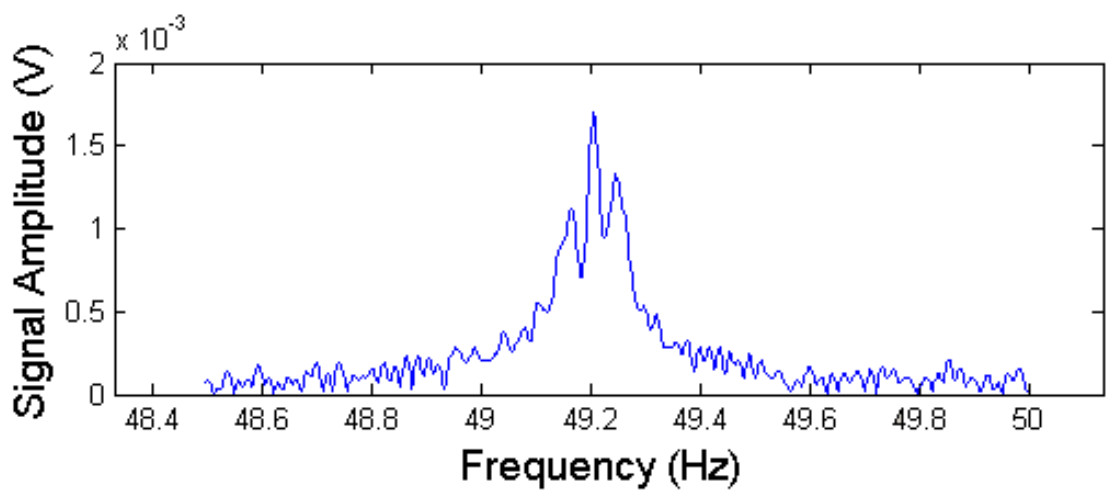

Figure 46. The asymmetry in the quadrupole-coupled ${ }^{131}$ Xe FFT becomes more exaggerated for lowamplitude tests like the delayed pulse method near the zero crossing. This causes difficulties for the fit function for ${ }^{131}$ Xe decay curves and thus more scatter in the overall delayed pulse curve.

To circumvent this issue we can simply take the first data point in the free induction decay to be the amplitude and ignore the fit altogether. Of course, we want to stick to using the fit whenever valid, but in situations such as this where the fit cannot find a reasonable solution, we usually end up with cleaner results by just ignoring the invalid fit. Table 6 and Figure 47 below compare the two analysis approaches for the same ${ }^{131} X e$ delayed pulse test. 
Table 6. Reported T1 values for ${ }^{131}$ Xe Delayed Pulse Method using Different Analysis Approaches

\begin{tabular}{|c|c|c|c|}
\hline Variable & Using Fit Amplitudes & Using Data Amplitudes & \% Difference \\
\hline $\mathbf{T 1}_{\text {fit }}$ & 20.24007 & 19.66101 & 2.902478 \\
\hline $\mathbf{T 1}_{\mathbf{0}}$ & 20.11638 & 19.98083 & 0.676123 \\
\hline
\end{tabular}
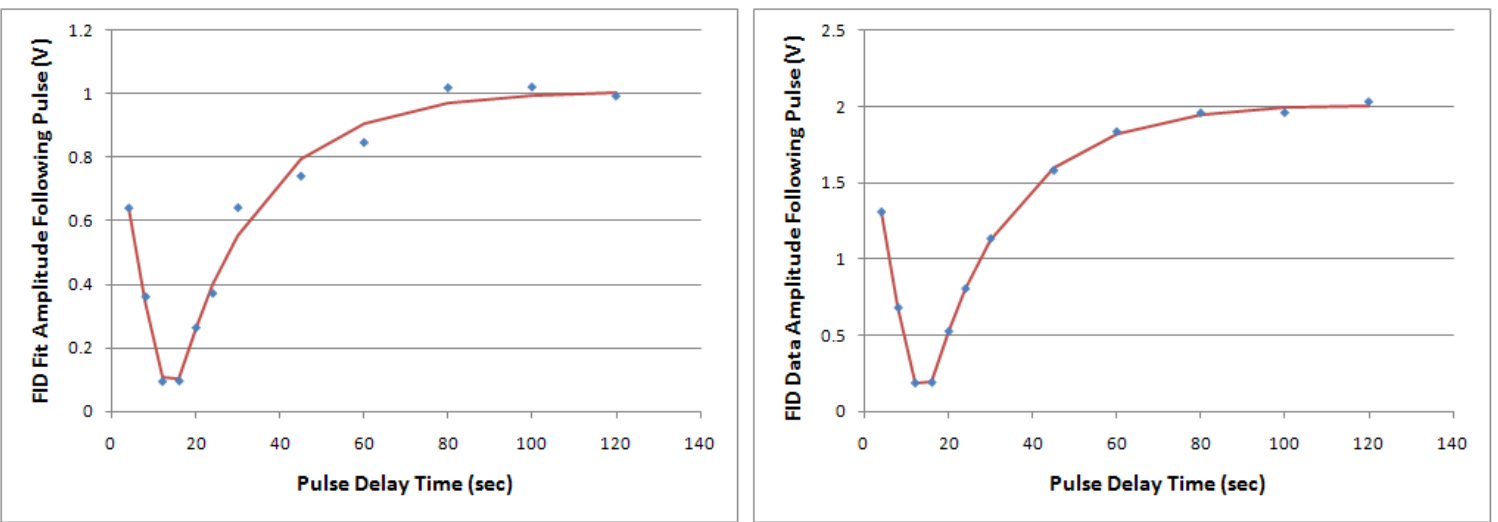

Figure 47. Comparison between analysis approaches for the delayed pulse method for ${ }^{131}$ Xe. On the left is the curve obtained by using the fit-reported amplitudes; on the right is the curve from simply taking the amplitude of the first data point in the decay curve for each delay run.

While the two analysis approaches yield very similar results, the curve obtained by taking the initial amplitude of the raw data (right in Figure 38) is clearly tighter and therefore assumed to be more reliable. Also note that the half-life based $\mathrm{T} 1_{0}$ values vary less than their fit-based counterparts, further supporting our decision to use $\mathrm{T} 1_{0}$ as the best option for the delayed pulse method.

The main drawback of the delayed pulse method is that it takes a long time to extract a value for T1. Whenever we apply a pulse, it takes time for the xenon group to return to steady state, and so we need to wait at least five T1 lifetimes between each delay run. Depending on the expected T1 value, it can take ten minutes to collect each data point, 
requiring at least 40 minutes to measure $\mathrm{T} 1$. This motivates us to collect as few data points as possible, which is not a typical sign of a good test method, so we would like to improve this situation.

Either way, that covers our industry standard methods for measuring both T2 and T1, and these methods have proven to be quite consistent and reliable. However, there are other methods that can work and can even be quite beneficial to us; they just have not been set up for use on our equipment specifically.

\section{B. New Methods for Measuring Spin Lifetimes}

Now, let us go over the basic premise for each and discuss some of the expected advantages and drawbacks of using these other methods before we get into their actual implementation.

\section{Measuring T2 Using the Stimulated Growth Method}

We already discussed the industry standard method for measuring T2 by monitoring the free induction decay following a $\pi / 2$ pulse. During that discussion we also covered the option of using a small-amplitude sustained drive instead of the pulse to stimulate the atomic precession and then pulling the drive at the start of the FID test. Well, what if we 
instead consider the beginning of the test to be the instant we first connect the smallamplitude sustained drive? In theory, the signal should grow up to some amplitude at which it reaches a steady state, and that growth should happen at a rate dependent upon $\mathrm{T} 2$.

We can look at this scenario in a similar light to the way we thought of the delayed pulse method; we essentially have two steady state conditions and we are interested in the rate of transition from one to the other. The difference is that in the case of the delayed pulse, both conditions output signal because we stimulate them in opposite polarization states. In this method and in the FID we have on one side the state where the atoms are polarized but not stimulated and on the other side the state where the atoms are fully stimulated. We already used equation 1 to describe the transition from stimulated to not in the free induction decay method, so we expect to use equation 5 to describe the transition going the other way.

If we have the system running (lasers and magnetic fields at the proper settings for NMR) but no transverse drive connected, then the xenon polarizations are aligned along $z$ but there is no precession about $z$ and therefore no signal. When we first apply the drive on $x$, all of the xenon atoms polarized along $z$ at that instant should begin to get stimulated and the signal should immediately grow up due to that. However, during the process of stimulation the atoms lose their coherence either by collisions which destroy their spin states or by passing through non-uniformities in the magnetic field. Eventually, they are 
again polarized through spin exchange with the alkali atoms, at which point they get stimulated once again.

So, in the first moments of the growth process it's all gain and almost no loss in stimulation, and thus the growth rate is fast. However, as time passes, atoms lose their spins and newly polarized atoms begin to get stimulated, adding to the overall signal but at a slower rate. This constant ebb and flow of the level of atomic stimulation should eventually reach a steady state where the rate of signal loss from spin destruction and decoherence is equal to the rate of signal gain from newly polarized atoms, and the signal profile from the total process over time should like the theoretical curve in Figure 48 below, generated by equation 5 .

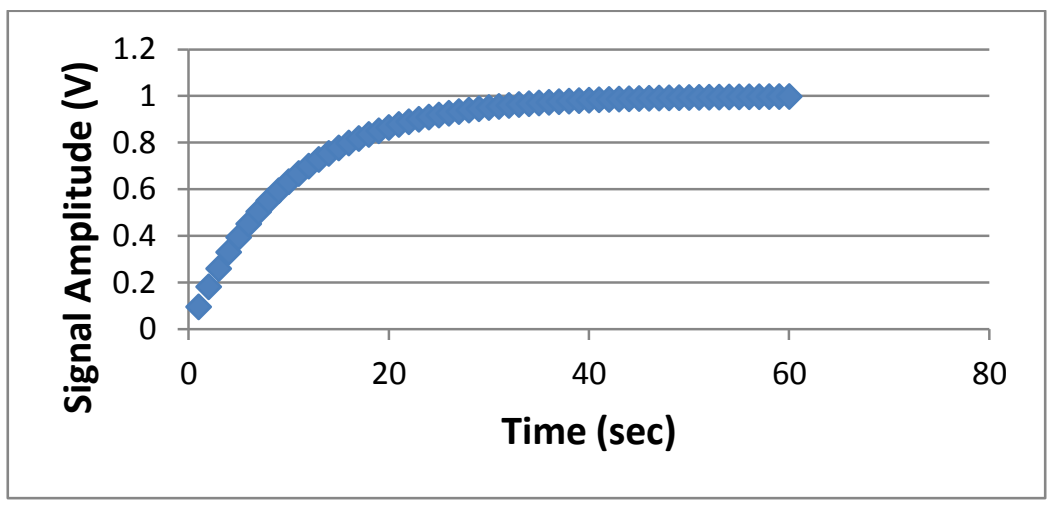

Figure 48. Ideal curve expected from the $\mathbf{T} 2$ growth method, constructed using equation 5.

We should be able to fit this curve using equation 5 and come out with a value for T2 just as we did for the FID method. 
The main advantage to this method would be that when we stimulate the atomic precession with a transverse drive, we force the atomic precession into the frequency of the drive, even if the drive does not exactly match the natural resonance. This will be an important tool for us as we will discuss shortly, but for now it means the drive should reduce or eliminate the quadrupole effects from the ${ }^{131} \mathrm{Xe}$ T2 test, which would speed up the analysis process slightly. On top of that, consistent successful demonstration of the growth method would provide a way to confirm our measurements from the FID method. If nothing else we can at least hope to gain further insight into the stimulation process.

Unfortunately, there are major complications in trying to run the growth method this way. At best, there is a great deal of inconsistency; often the shapes do not quite match from run to run, and usually the fit function cannot find a reasonable solution. Some example data sets from failed T2 growth tests are presented in Figure 49 below.

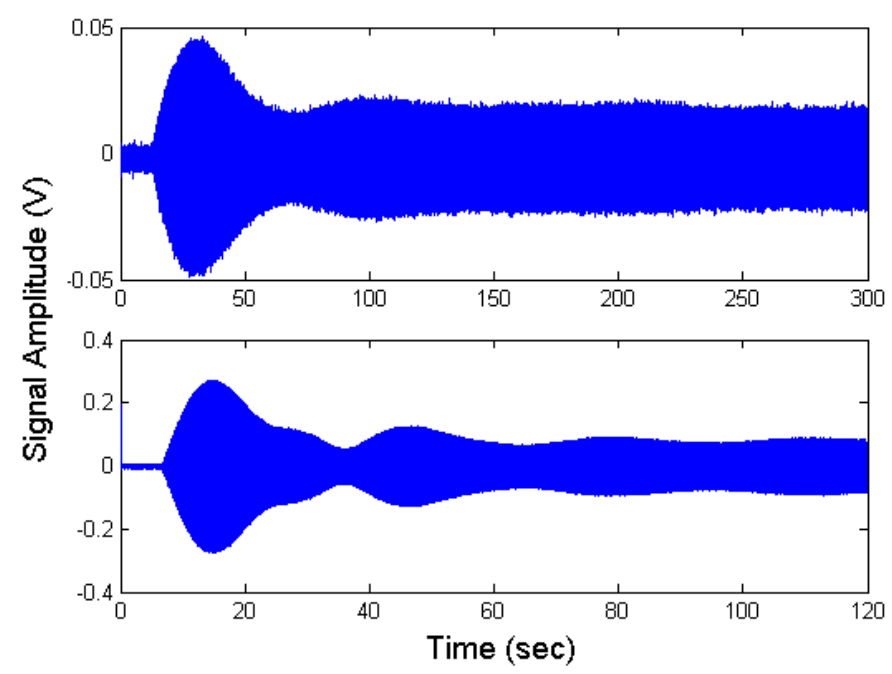

Figure 49. Example data sets exhibiting complications in the T2 growth method. 
Clearly, these look nothing like the trend described by equation 5, and these are not fluke mishaps but quite typical types of results. Even if we obtain exceptionally clean data (after many failed attempts), it usually looks something like the data in Figure 50 below. Although the first 50 seconds or so worth of data seem to follow equation 5 fairly well, there are certainly defects in the curve. The fit solution shown in the third chart returns a T2 value within one second of the FID results, which is indeed encouraging, but we still cannot justify using this test as a competitor to the FID T2 method.
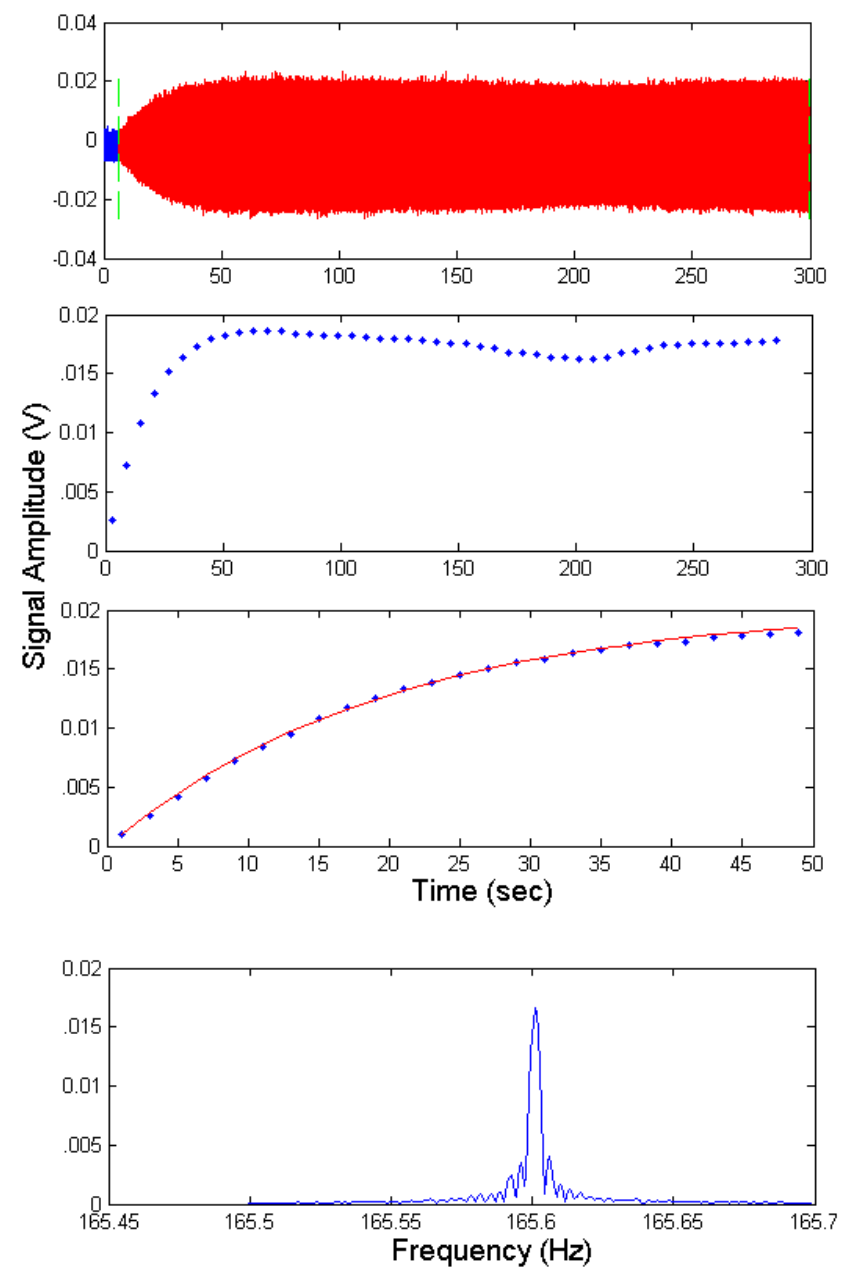

Figure 50. An exceptionally well behaved $T 2$ growth test still exhibits very clear shape deformities. 
To better understand the odd behavior of this method, we must explore the effects of changing some of the test conditions. The most obvious choices to begin with would be the drive parameters, namely the amplitude and frequency, since the stimulating drive is the newly introduced portion of the method.

\section{a. Shape Inconsistencies in the Growth Method}

The most common and frustrating source of inconsistency is that it is impossible to match the natural frequency exactly with the drive (at least with our open-loop test setup). If the frequency does not match the natural atomic resonance, the process of stimulating atomic precession is slightly more complex.

As soon as the drive pushes a xenon atom's spin off the $z$ axis, the atom immediately begins its natural precession about $z$ at frequency $f_{\text {Larmor }}$ based on the magnetic field strength. Over time, the drive pulls the atomic precession into its own frequency $f_{\text {drive }}$ and during that process both frequencies are present in all stimulated atoms. This process is difficult to visualize in three dimensions, but essentially the atom accumulates some wobble due to the discrepancy between $z$ DC field strength and drive frequency. The two frequencies will interfere with one another as the signal passes through the demodulator, adding to the overall amplitude when the two frequencies match in phase and subtracting when they oppose. This results in beating in the signal at a period of 


$$
T_{\text {beat }}=1 \Lambda f_{\text {drive }}-f_{\text {Larmor }} \mid \text {, }
$$

which will be very important to us shortly. For now, it explains some of the effects we see in the failed tests. The farther off the natural resonance we drive, the faster the beating.

The other pitfall to avoid is that the drive amplitude has a threshold above which the theory behind this method breaks down. If we drive too hard, we can actually temporarily push the atomic stimulation so far before collisions and magnetic field non-uniformities become a significant factor that the signal overshoots the steady state amplitude and then slowly settles back down. This probably partially explains the strange shape of the profiles in Figure 49 above. This also introduces insurmountable problems for our fit function. We avoid this issue by maintaining low drive amplitudes, which severely limits our SNR capabilities in this test and causes more random scatter in our data profiles.

All in all, the sustained drive complicates things, which is unfortunate since all three of our new test methods require it. However, we can craft quite a useful tool by intentionally driving off the resonance frequency. 


\section{b. Offsetting the Drive Frequency to Eliminate Beating}

If we perform a $\mathrm{T} 2$ growth measurement with the same procedure as discussed above but simply set our drive frequency to be something like $0.5 \mathrm{~Hz}$ higher than the natural Larmor frequency, we get data like that shown in Figure 51 below.

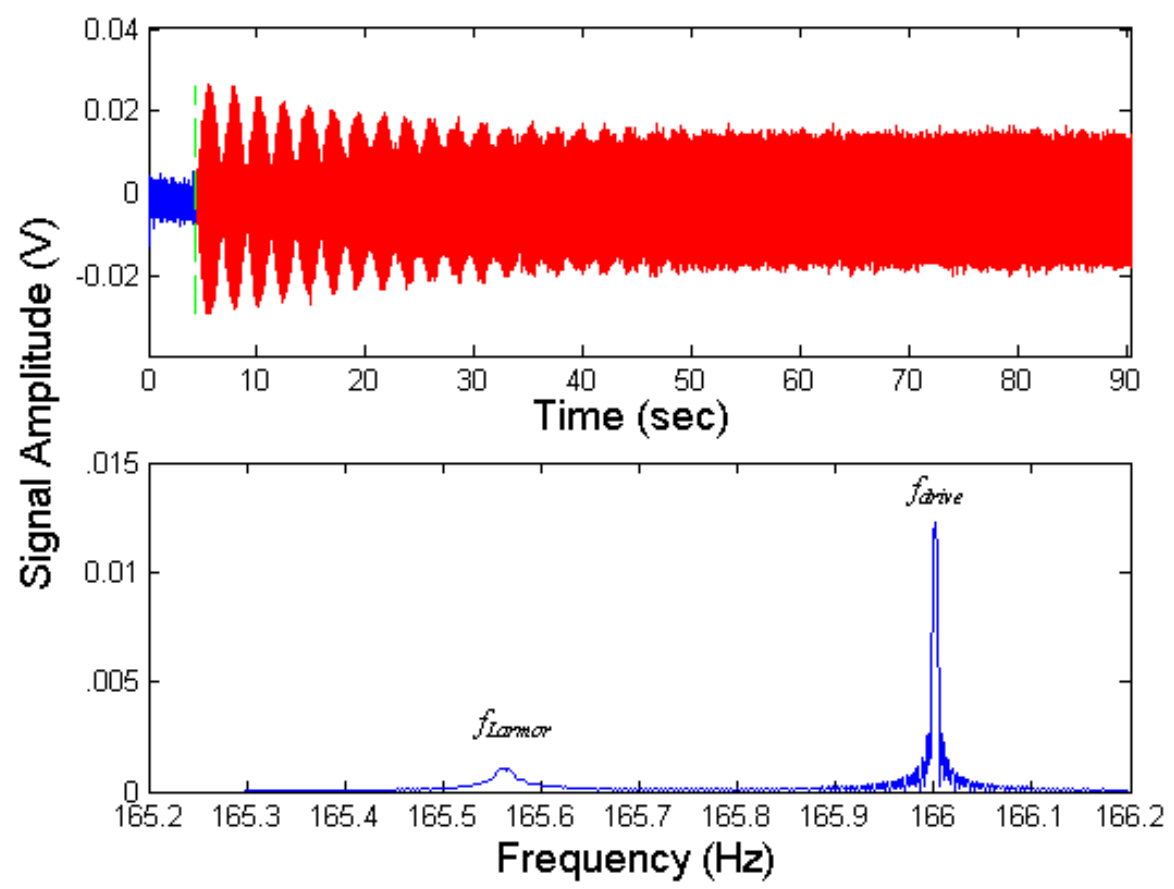

Figure 51. Raw data from a $T 2$ growth test with intentional drive frequency offset.

The FFT displays the two distinct frequencies, and the relatively high-frequency beating is quite apparent in the raw data. Notice that the peaks of the beats form a sort of decay curve throughout the run, and the valleys a growth curve. However, if we use an arbitrary window size, like our usual 0.5 seconds, it does not matter which frequency we choose to create our data profile; we end up with the useless scatter shown in Figure 52 below. 


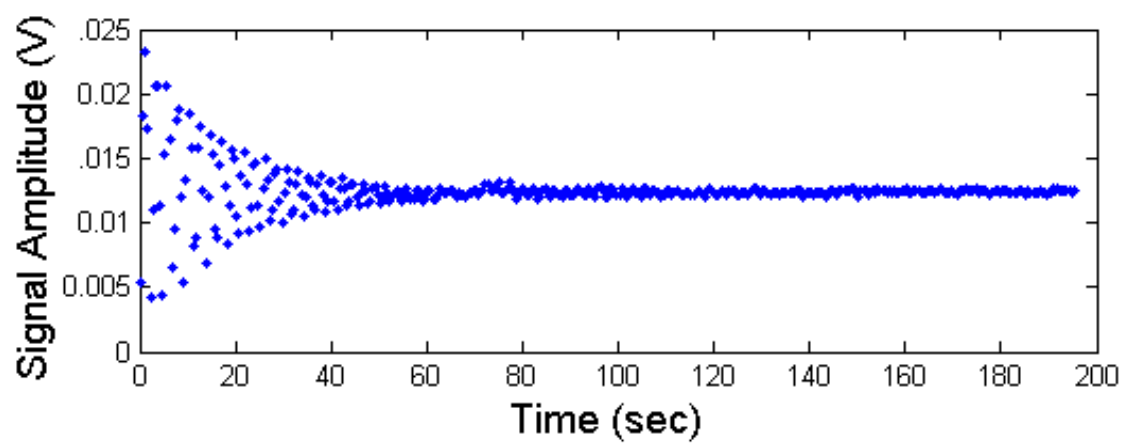

Figure 52. Signal profile using 0.5 seconds window size from the raw data in Figure 42 above.

Now, what we see here is the decay of the beating itself as the signal from the natural precession diminishes, but there is no obvious order to it and clearly we cannot fit a profile like this with equation 1 or equation 5 . The trick is to set our window size equal to the beat period so that each data point in our profile represents the amplitude of a single beat. We determine the two frequencies from the FFT and plug them into equation 8 to find our window size of

$$
T_{\text {beat }}=1 / 166.0013 \mathrm{~Hz}-165.5619 \mathrm{~Hz} \mid=2.27583 \text { seconds, }
$$

which we use to generate two profiles: one focused on the amplitude of $f_{\text {Larmor }}$ throughout the run and one on $f_{\text {drive }}$, both of which are displayed in Figure 53 . 


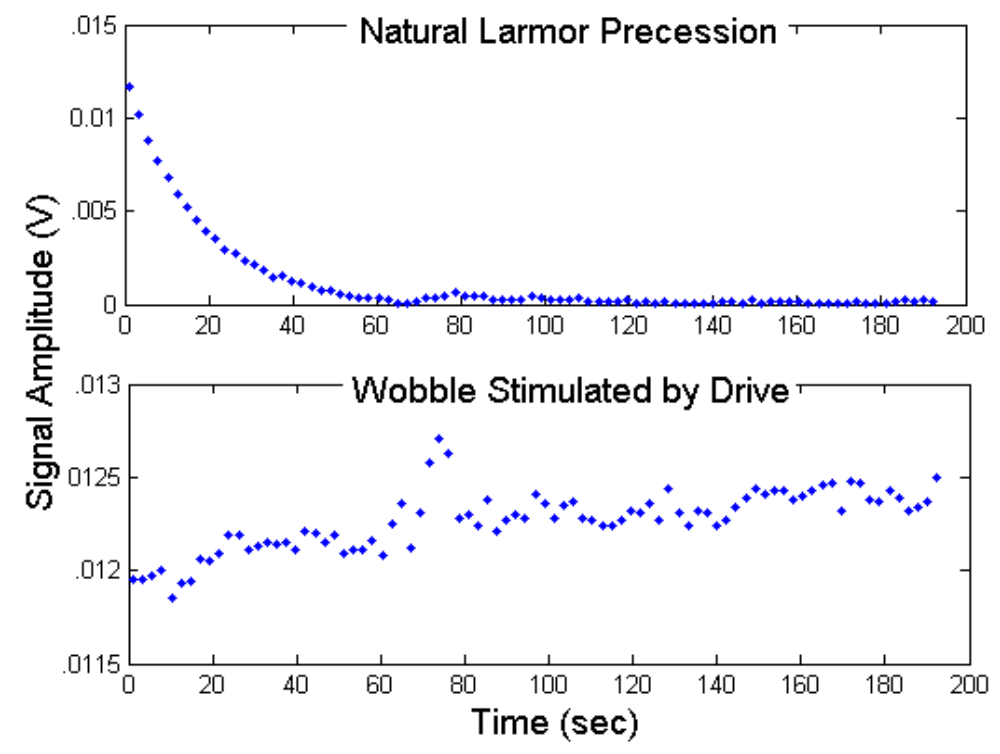

Figure 53. Signal profiles of data from Figure 51, generated using window size of $\mathbf{T}_{\text {beat }}$ focused on $f_{\text {Larmor }}\left(\right.$ top) and $f_{\text {drive }}($ bottom).

Now we have gotten to the heart of the matter. There are several key pieces of information to gather from these two profiles. First, notice that the natural precession decays down to zero, following quite a nice free induction decay curve (hopefully governed by T2, which we will get to shortly). Second, notice that the stimulated precession remains practically constant in amplitude, and what's more, maintains roughly the same amplitude that the natural precession begins with. This explains the behavior of the raw data. When the amplitudes of the so-called wobble and the natural precession are equal at the start of the test, the signal nearly cancels out in destructive interference and doubles in constructive interference; when the natural precession has disappeared, we are left with the constant-amplitude driven precession at the end of the run. 
So it seems that somehow the supposed growth is actually the result of subtracting the signal of the natural precession from that of the driven precession. We could probably get away with fitting the natural decay using equation 1 but since we had set out to use the growth method, we may as well go all the way and generate our growth curve by subtracting the two profiles. We then fit it using equation 5, as shown in Figure 54 below.

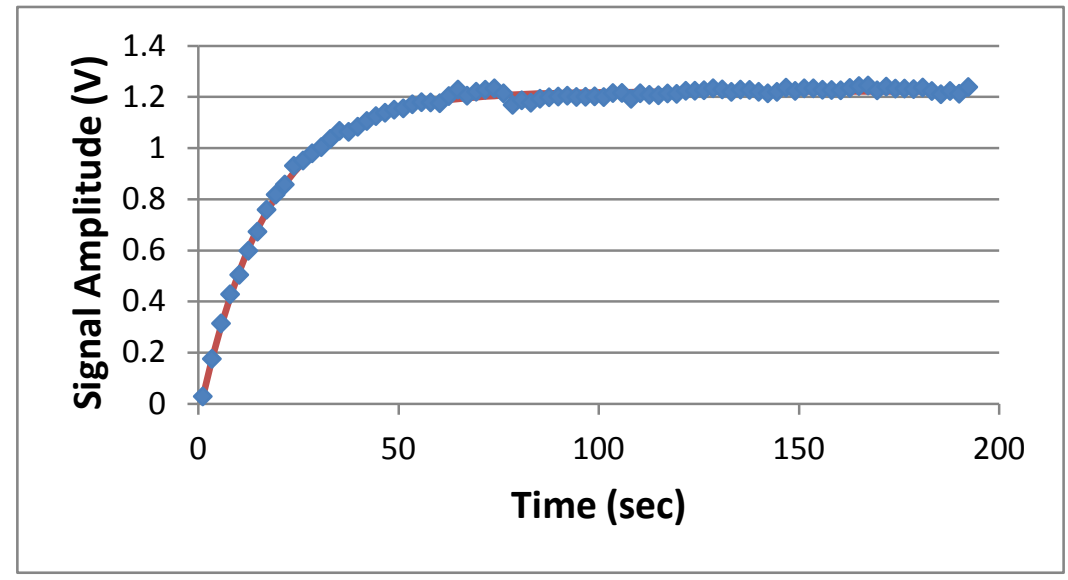

Figure 54. Fitted growth of driven precession matches T2 from FID within $10 \%$

We would like to get an idea of the statistical consistency of results when using this method. To do so, we perform this growth test using the same drive frequency five times, and we also perform an FID test by pulling the injected drive between each of these five tests. Now we can compare the two methods using the same setup parameters for both. We find the results presented in Table 7 below.

Table 7. Comparison of example results between growth and decay methods for measuring T2

\begin{tabular}{|c|c|c|}
\hline Method & T2 Value & Statistical Uncertainty \\
\hline Growth & 20.26597 & 0.389839119 \\
\hline Decay & 19.65804 & 0.178888498 \\
\hline
\end{tabular}


Comparing statistical uncertainties, the growth method has about half the precision of the decay method; if we take the $\mathrm{T} 2$ decay measurement to be our true value, the $\mathrm{T} 2$ growth measurement is about $3.1 \%$ off. To be sure, the growth value still seems to be a well behaved measurement, but we have no reason to favor it.

Now, it seems strange that we cannot monitor any physical signal growth but rather must construct it ourselves from the two separate profiles. Quite possibly, our visualization of the physics behind this method is slightly incorrect or incomplete. Still, this approach is sufficient to present the extremely important technique of stimulating precession by intentionally driving off the natural resonance, the significance of which will stand out prominently in our discussion of the next test method, the Flipped Polarization method. Before we use it for that, however, we should first try to understand the limits and consequences of doing so. Most importantly, what happens to our results as we drive farther off resonance, and are the effects symmetric about the natural frequency? We investigate by performing a series of growth measurements using various drive frequencies, the offset from the natural resonance sweeping from roughly $-1.0 \mathrm{~Hz}$ to +1.0 $\mathrm{Hz}$; the results are shown in Figure 55. Anything much farther off resonance will not stimulate enough signal to work with. 


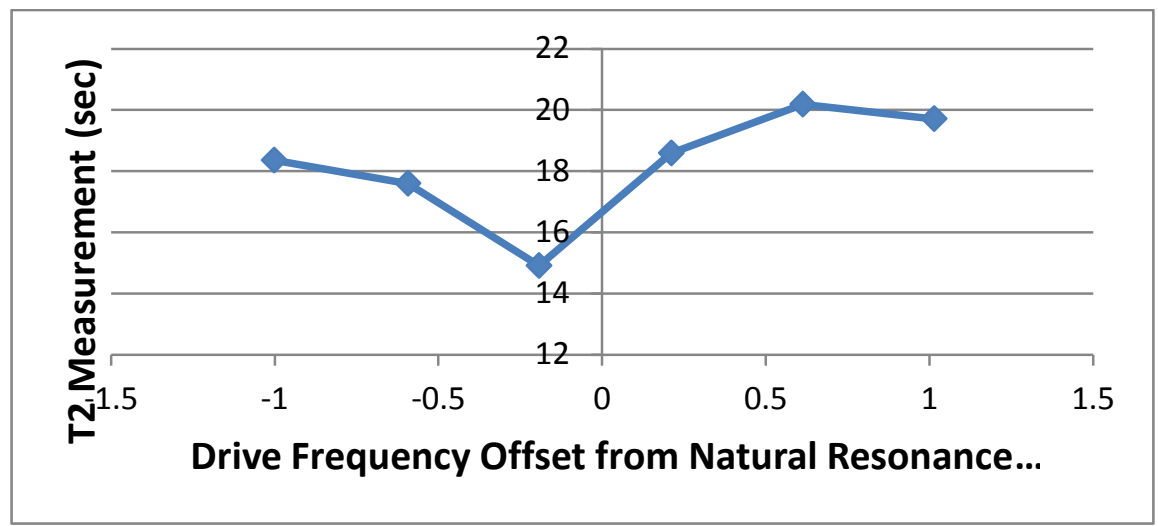

Figure 55. T2 growth measurement dependence on drive frequency offset.

The drive frequency offset certainly seems to have an effect on the results. The statistical uncertainty for these six measurements is about 0.839 seconds, which is more than double the uncertainty associated with repeating the measurement for a single drive offset as was presented in Table 7. Now, the true value of the $\mathrm{T} 2$ here may not necessarily be the 19.66 seconds determined by decay measurements earlier, given that this test was performed on a different day under different tune-up conditions. Still, the only regions of drive offset that seem to yield relatively consistent restults are out on the wings, when the offset is large. Under such conditions, the measured T2 values seem to converge towards something like 19 seconds.

It is possible that the large errors associated with the small-offset region may be due to overstimulation; since the drive is much more effective when closer to resonance, perhaps the drive amplitude was too high to yield accurate results in that region. Secondly, as described by equation 8 , the closer to resonance we drive, the larger we must make our window size, which limits our data resolution and the effectiveness of the fit function. So, as a rule of thumb we may as well try to use a fairly large drive offset 
when performing this type of test; usually we drive somewhere between 0.3 and $0.6 \mathrm{~Hz}$ above the resonance frequency.

Before we move on to the next test, we want to make note that our hope for this method to eliminate quadrupole effects in ${ }^{131} \mathrm{Xe} \mathrm{T} 2$ measurements will unfortunately not be realized using this approach. Since we construct our growth curve directly from the natural decay curve, we end up with the same quadrupole beating in the growth curve as in the decay curve, as shown below in Figure 56.

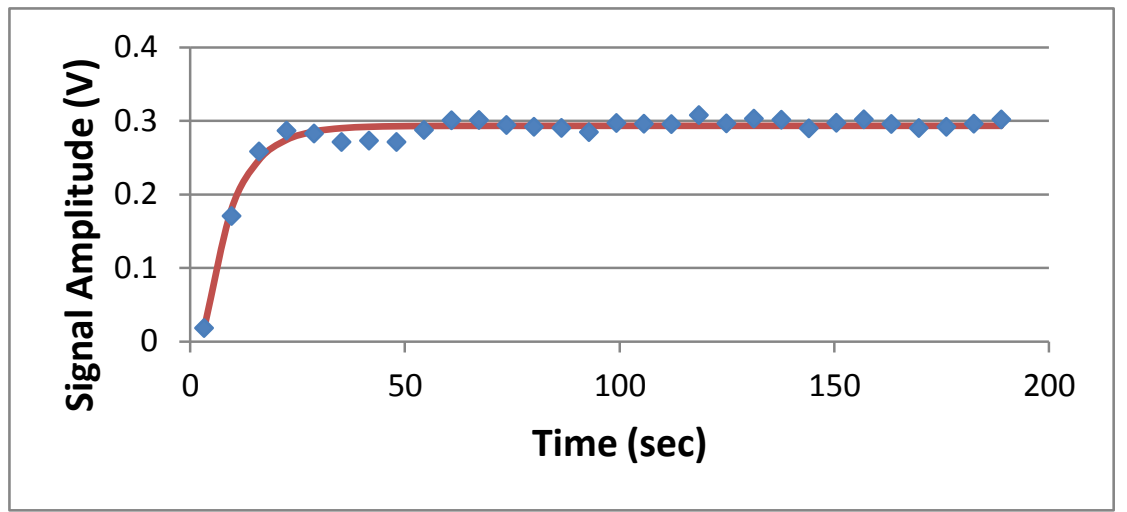

Figure 56 Growth method used to measure ${ }^{131}$ Xe T2. The quadrupole beating effects are not eliminated using this approach to the growth method.

Even though in this case we can still manage to fit the growth curve without including the quadrupole description, we have gained no advantage over the FID method. On top of that, the analysis required to construct the growth curve takes far longer than the FID method, and it is more difficult to determine the test start time, so we really have no justification to use the growth method as our primary T2 measurement technique. 
Still, we have gained some truly valuable experience from this method; plus, our real motivation in exploring these new test methods at all is to find a faster way to extract T1. The following two methods, if demonstrated successfully, could reduce the testing times needed for T1 measurements by as much as an order of magnitude.

\section{Measuring T1 Using the Flipped Polarization Method}

With the Delayed Pulse Method, we have to perform an entire data collection run (including the settling time in between each run, a minimum of five T1 lifetimes) for every single data point. With a bare minimum of four points needed to make a conclusive measurement, our industry standard T1 test usually takes at least 20 minutes for each isotope, whereas the Flipped Polarization Method could potentially yield a more precise measurement in a single run, taking at most a few minutes. As we look to the future and the prospect of mass production of high-quality NMR gyro technology, reducing cell testing times will become very important. From a science perspective, faster results provide the means to develop statistical data using repeated measurements as well as the ability to study drive transience characteristics more effectively. In other words, it would be a win-win situation to get this method running properly.

The basic idea of the flipped polarization method is to try to monitor the xenon shift from one polarization to the other in real time. There are actually two ways that we should be able to accomplish this. Our original idea involves altering the polarization of the pump 
laser. The light from the laser is linearly polarized before passing through a quarter-wave $(\lambda / 4)$ plate, angled appropriately, such that the outgoing pump light is circularly polarized. Turning the quarter-wave plate 90 degrees reverses the polarization of the outgoing light (from $\sigma^{+}$to $\sigma^{-}$or vice versa), as illustrated in Figure 57 below.
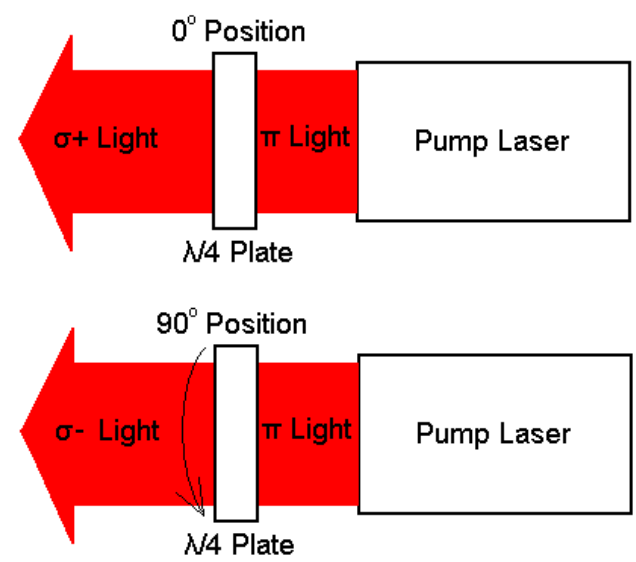

Figure 57. Rotating the quarter-wave plate 90 degrees reverses the direction of circular polarization of the outgoing pump laser light.

This in turn reverses the polarization of the alkali atoms and, through spin exchange, that of the xenon as well. We will refer to this implementation as the quarter-wave plate approach. A later-discovered implementation of the flipped polarization method keeps the lasers untouched and instead utilizes the $\pi$ pulse to flip the xenon spins; this we will call the $\pi$ pulse approach. 


\section{a. The Quarter-Wave Plate Approach}

It should be noted that the polarization direction of the alkali vapor measurably affects the $z$ magnetic field and thus the xenon precession frequencies. Fortunately, the alkali pumping process reaches steady state in a matter of milliseconds at most; the xenon lifetimes we are measuring are 3 to 5 orders of magnitude longer than that, so we can presumably neglect the transience of the alkali in the very start of the test.

To begin the test, we expect we'll want the system tuned up as usual, with the $z$ fields set for a specific alkali orientation and the transverse drive frequency set to match the xenon Larmor frequency for a specific laser polarization, say $\sigma^{+}$light. Once everything is tuned properly, we prepare the test by rotating the quarter wave plate so that the pump laser now emits $\sigma^{-}$light. We wait several minutes (at least five T1 times) to ensure that nearly all of the polarized xenon atoms are spin down before the test. Finally, after sufficient waiting, we begin recording data, wait a few seconds, and then quickly rotate the plate back to its original orientation.

We expect to see the xenon signal grow at first, since the alkali polarization now generates the correct magnetic field such that the natural xenon precession matches the $x$ drive frequency much more closely. Shortly thereafter, however, the signal should begin to look like a T1 delayed pulse curve; the alkali atoms, now spin-up from the newly reintroduced $\sigma^{+}$light, influence the longitudinal spins of the xenon atoms which then begin to cancel each other out. As usual, the signal should eventually grow back up as the 
spin-up xenon population begins to outweigh the spin-down. The theoretical process is illustrated below in Figure 58.

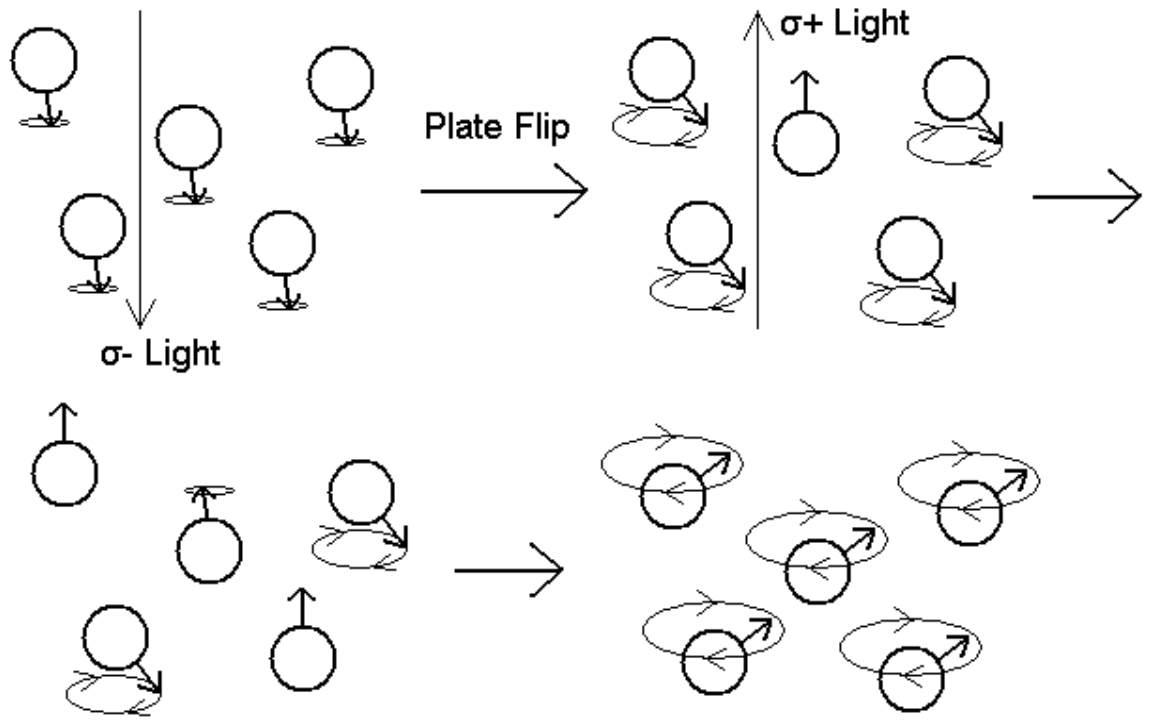

Figure 58. Visual representation of the quarter-wave plate approach to the flipped polarization method. The test is prepared with $\sigma^{-}$light until steady state precession is achieved. The quarter-wave plate is then flipped, introducing $\sigma^{+}$light and altering the polarization of the alkali. The xenon stimulation increases for the still negatively polarized atoms as the magnetic field from the alkali now brings the natural xenon precession frequency up to match the drive frequency. Meanwhile, the positively polarized alkali begins spin exchange with the xenon, resulting in simultaneous stimulated growth and polarization shift in the xenon population.

Unlike the delayed pulse method, in which our only indication of xenon spin populations comes from a $\pi / 2$ pulse sometime in the middle of the transition process, we can now monitor the relative populations of the xenon spin groups throughout the whole run and watch the shift in longitudinal polarization in real-time, including the zero-crossing 
marking the apparent half-life. Below in Figure 59 is an example data set for an attempt at this test method.
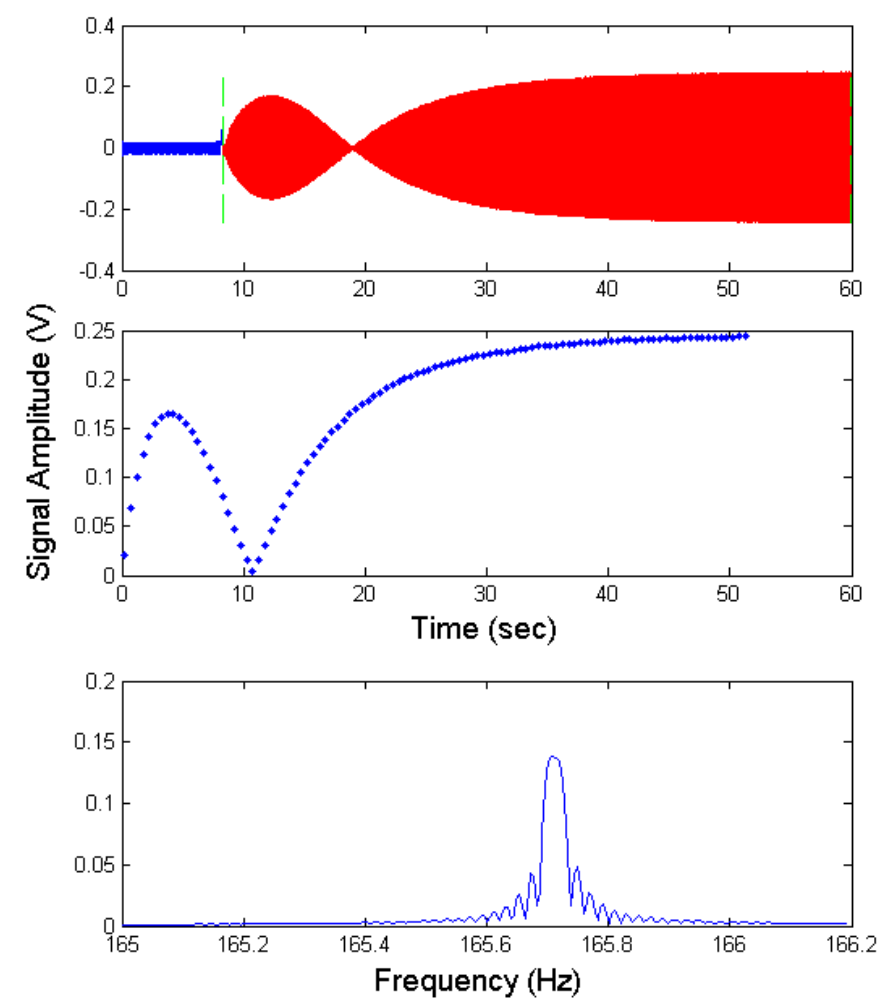

Figure 59. Typical data for the Flipped Polarization method by rotating the quarter-wave plate. As usual, the raw data is accompanied by the signal profile and the frequency-spectrum data used to create it.

Notice that in the beginning of the run before the plate flip, there is a small but still noticeable signal due to the off-resonance stimulation. As we expected, once the plate is flipped and the alkali vapor returns to the spin-up state, the drive frequency matches the xenon precession frequency much more closely and we see the signal grow up immediately before decaying due to longitudinal spin cancellation. 
Also notice that the FFT does not display a clean peak. This is most likely due to the drive being slightly offset from the natural Larmor frequency. However, it is possible that the xenon polarization level is high enough to also measurably affect the $z$ magnetic field (to a much lesser extent than the alkali), in which case the precession frequency would shift slightly as the xenon polarization changes. Whatever the cause, we cannot fix or eliminate the frequency spread for now, so we merely keep it in mind.

Now, if this method simply worked as we first expected, we should be able to calculate T1 by plugging the apparent half-life at about 10.9 seconds into equation 2 to find a longitudinal lifetime of about 15.7 seconds.

The bad news is the curve obtained from the delayed pulse method using this same cell for the same xenon isotope at the same temperature, shown below in Figure 60.

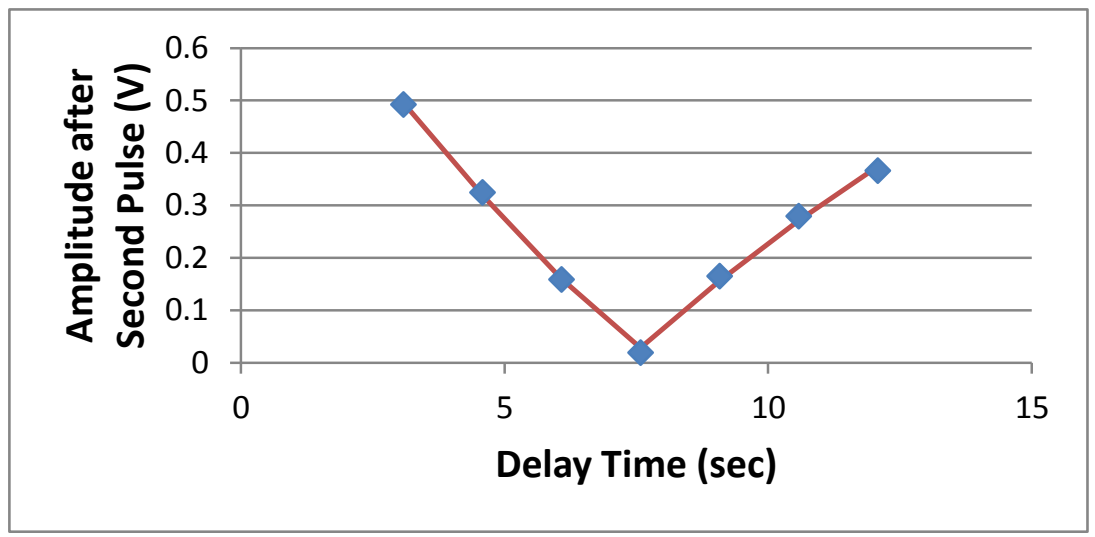

Figure 60. The delayed pulse curve exhibits an apparent half-life much lower than that of the flipped polarization curve in Figure 50. This discrepancy between the two methods is very common but not consistent in magnitude. 
Disappointingly, this implementation of the flipped polarization method almost always exhibits a half-life higher, usually significantly so, than the delayed pulse results. In this example, the delayed pulse yields a lifetime of 11.2 seconds, a discrepancy of about 40.2\%. To add to the frustration, we observe little to no consistency in the magnitude of the difference between the results from the two methods from cell to cell.

Still, if our understanding of the physics is correct, we should be able to fit the profile in Figure 50 by combining the $\mathrm{T} 2$ and $\mathrm{T} 1$ growth curves, or in other words multiplying equations 5 and 6 . This gives us

$$
y=A^{*}\left|e^{\wedge}(-t / T 1)-V\right| *\left[1-e^{\wedge}(-t / T 2)\right]+v
$$

for our fit function. After much trial and error for initial fit guesses, Figure 52 below shows the profile with the solved fit overlaid, and Table 8 shows the fit parameters associated with the solution.

Table 8. Values used to generate fit solution in Figure 61 below.

\begin{tabular}{|c|c|c|}
\hline Variable & Value & Units \\
\hline $\boldsymbol{A}$ & 1261.344 & Volts \\
\hline $\boldsymbol{T} 2$ & 8.146046 & Seconds \\
\hline $\boldsymbol{T} \boldsymbol{1}$ & 6.436635 & Seconds \\
\hline $\boldsymbol{V}$ & 0.190417 & Volts \\
\hline $\boldsymbol{v}$ & 10.43908 & Volts \\
\hline
\end{tabular}




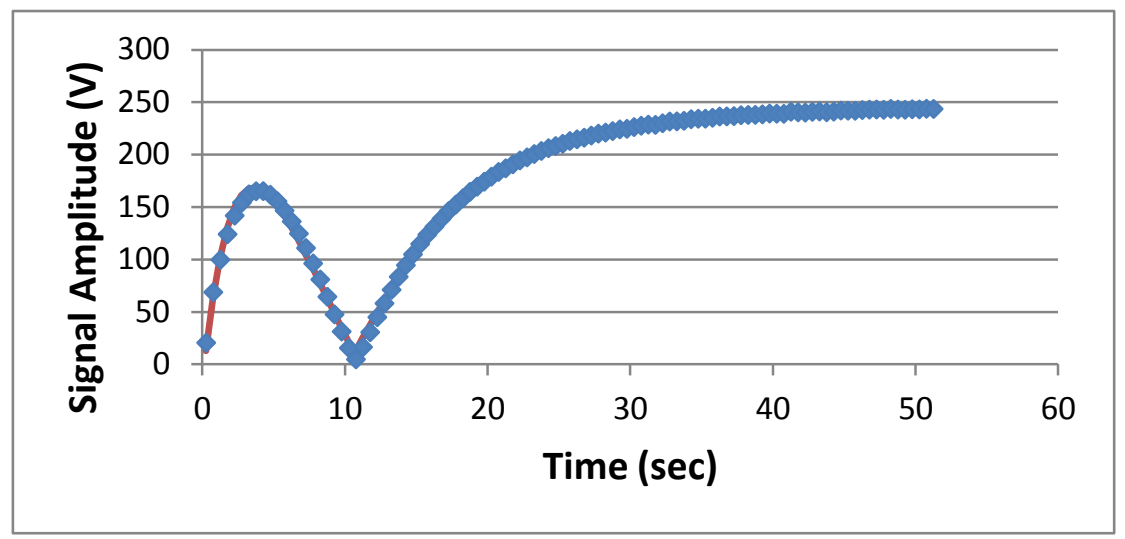

Figure 61. Fitted curve generated by the quarter-wave plate approach to the flipped polarization method. The parameters used to generate the fit solution are listed in Table 8 above.

While the fit function seems to be able to match the shape of the curve fairly well, there are several huge red flags. First of all, notice that the T2 value is larger than the T1, a physical impossibility. Second, the solved T1 value is $42.8 \%$ lower than the delayed pulse value, so not only is it still far off but it is also extremely inconsistent with the apparent half-life. Third, note that the relationship of $A$ to $V$ is far different from the 2:1 ratio we like to see for the delayed pulse curve. These are all warnings suggesting that, even though the fit looks pretty good, it is in fact useless to us as is. At best we can hope the problem lies in our experimental approach, and not in our fit function.

Fortunately, we can test this hypothesis because there is a second approach to the flipped polarization method - the $\pi$ pulse approach - of which we conceived well into our investigation of the flipped polarization method. 


\section{b. The $\pi$ Pulse Approach}

Of course, the goal remains the same, but the implementation is far simpler. In the plate approach we have to deal with the intrinsic T2 growth associated with the change in magnetic field strength, not to mention any inconsistencies in the physical rotation of the plate. In this approach we never alter the polarization of the light or the alkali. Instead, we allow steady state stimulation as usual and then, shortly after initiating data collection, we use a $\pi$ pulse to flip the xenon spins. This preserves any level of stimulation the xenon atoms had at the time of the pulse; it simply reverses the spins, as shown in Figure 62 below. The atoms then begin returning to the initial polarization and are again stimulated by the drive, as we are now quite used to seeing.

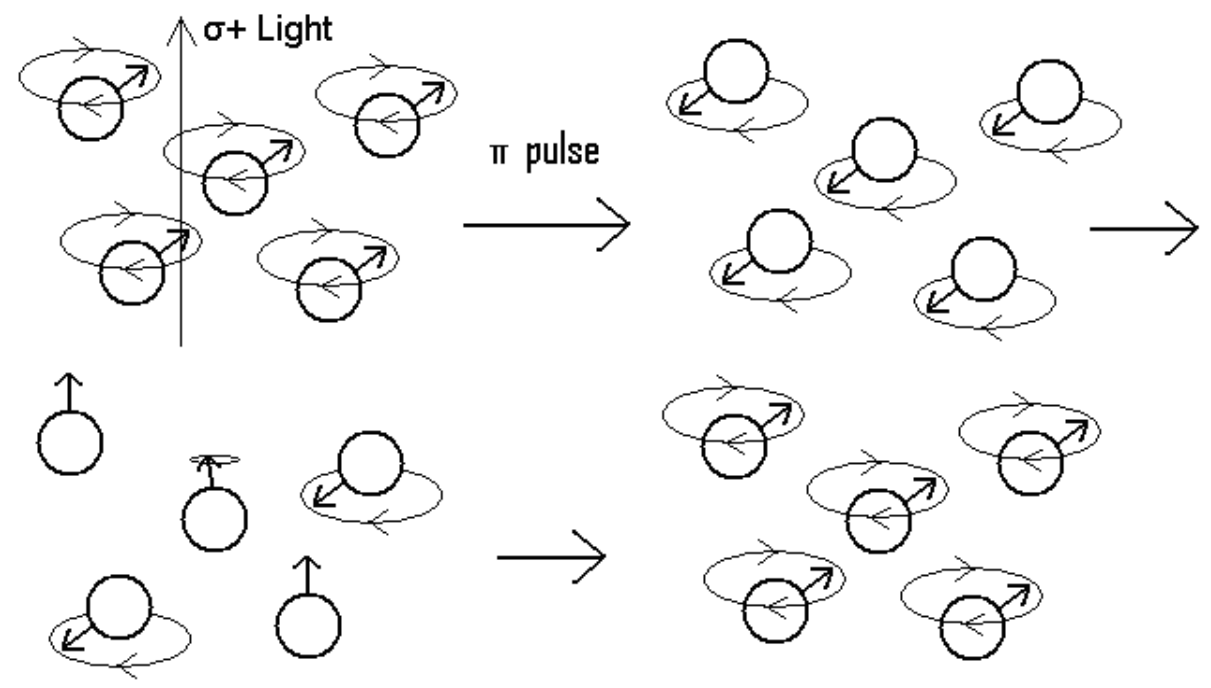

Figure 62. Visual representation of the pulse approach to the flipped polarization method. With the atomic precession in steady state from the drive, a $\pi$ pulse is applied which flips all of the polarized xenon spins by $180^{\circ}$. As time passes, the xenon atoms return to positive polarization through collisions with the pumped alkali and are again stimulated by the drive. 
To implement this method, we must inject the small transverse drive on $x$ as usual, on which we usually apply the pulse as well. What we realized is that for this test, the pulse can be injected on $y$. Of course, this means it is picked up very strongly in the data, but it should not matter since all of the data of interest comes after the pulse. An example data profile using this approach for the same cell and isotope is shown below in Figure 63.

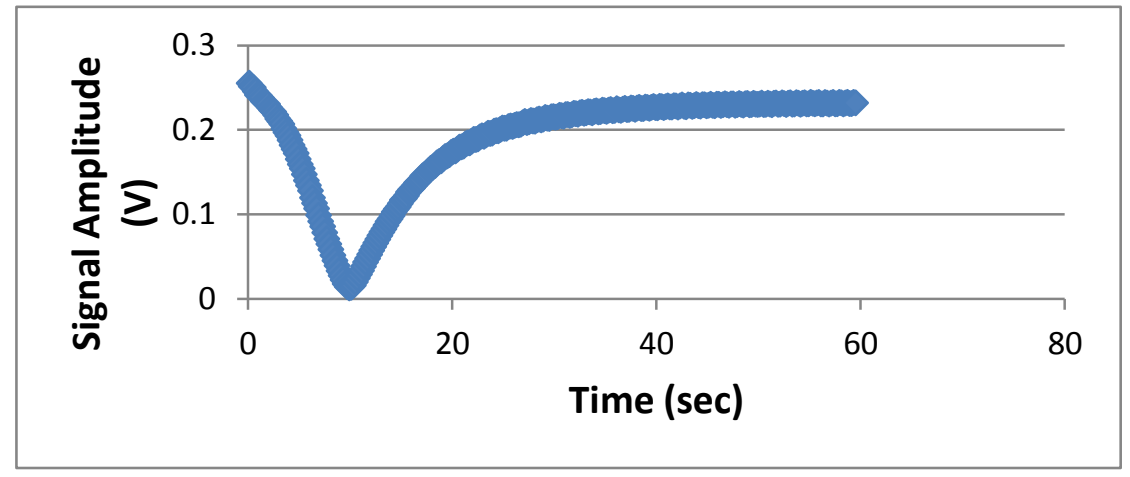

Figure 63. Data profile obtained using the pulse approach to the flipped polarization method.

This curve looks much more similar to the delayed pulse curve, but it still has some strange behavior at the start. Most likely, the $\pi$ pulse used was not exactly correct, causing slightly higher stimulation at the start which decays throughout the run. Still, it is somewhat comforting that the apparent half-life is quite similar between the two approaches; the method as a whole seems to be self-consistent. Whatever is causing the discrepancy in apparent half-life between this method and the delayed pulse method is a real effect and is universal across the two approaches to the flipped polarization method. We can investigate a little further by repeating the two approaches with a lower amplitude drive to see what, if any, effects the level of stimulation has on the shapes of the curves. The two profiles for these repeated tests are presented in Figure 64 below. 


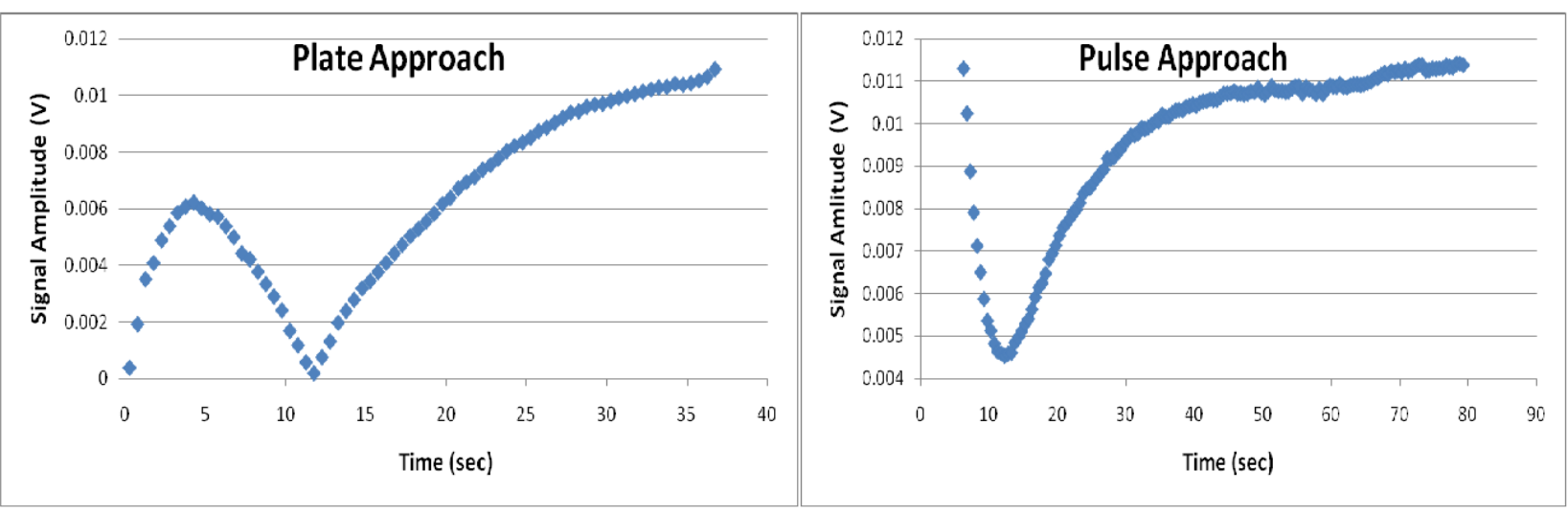

Figure 64. The two approaches to the flipped polarization method repeated with a low-amplitude drive

We often see shape deformities near the region of the zero crossing when the cross-axis rejection is low. It is impossible to align the coil axes perfectly, and so for these tests involving the transverse drive it is unfortunately common for the usually negligible $y$ component of the $x$ drive to bleed through on the Faraday detection. Still, even when the data never hits a clear zero we can usually estimate the half-life fairly well.

The important thing is that the apparent half-life seems unaffected by the drive amplitude, further convincing us that the $\mathrm{T} 1$ effects we are measuring here are real and consistent. The drive amplitude does seem to affect the shape of the pulse curve, however, which leads us to believe that the problem has to do with our understanding of the T2 effects or at least our representation of them in the fit function. Also, for both cases the SNR is clearly much lower as the scatter is more noticeable, as is the low-frequency beating from the inevitable slight frequency offset. 
For a long time I was stuck at this point, clearly able to see the shapes we expect but rarely finding results consistent with the delayed pulse method. I went to great lengths to verify every step of the experimental procedure and the analysis of the data several times. I even tried using the raw data profile instead of that constructed from the FFT amplitudes and found no improvements. I was close to giving up on ever finding a solution, and then I discovered the technique of driving off resonance, as we discussed for the T2 growth method.

\section{c. The Off-Resonance Flipped Polarization Method}

Recall, from the $\mathrm{T} 2$ growth example, the data profile constructed by focusing on the drive frequency in Figure 44; the amplitude of the profile shoots immediately up to roughly the steady state value and remains there throughout the test. We take this behavior as an indication that we can eliminate most of the $\mathrm{T} 2$ effects by driving off resonance and constructing the profile from the drive frequency. Of course, for the growth method the T2 is the only value of interest so eliminating its effects would have been useless; here, on the other hand, we would certainly prefer to look at a pure T1 curve to see if that resolves our problem.

We continue on, using a new cell whose delayed pulse results are shown in Figure 65 below. 


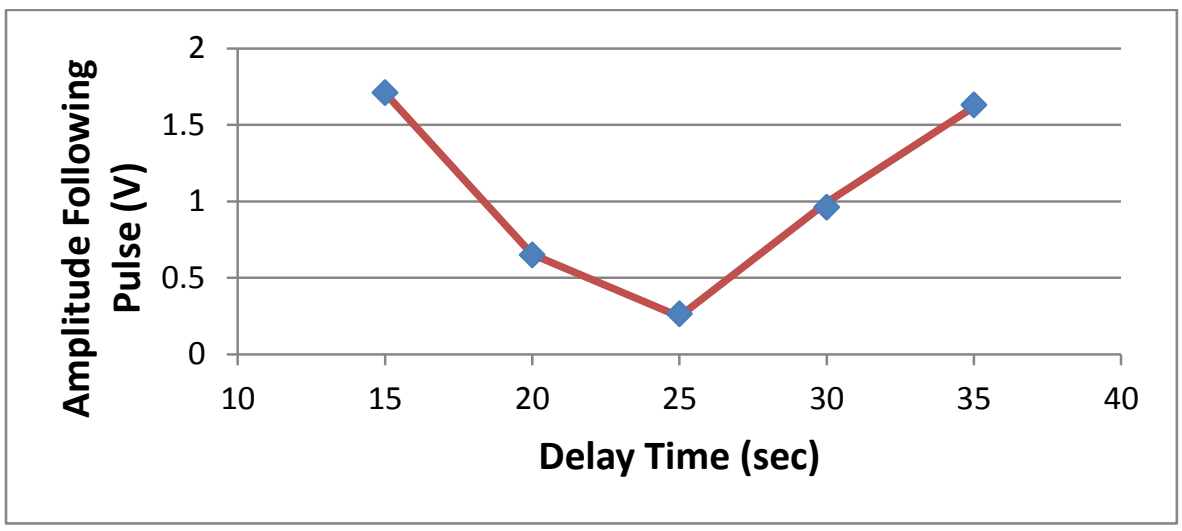

Figure 65. Delayed pulse curve for current cell on which to perform off-resonance flipped polarization tests.

So now we know to look for a zero crossing of about 24 seconds when trying to determine the success of the flipped polarization method using the new off-resonance drive technique. We perform both the plate approach and the pulse approach to the test, this time setting the drive frequency to $165.8 \mathrm{~Hz}$, about $0.2 \mathrm{~Hz}$ higher than the natural resonance. The data from both tests are shown in Figure 66 below. 

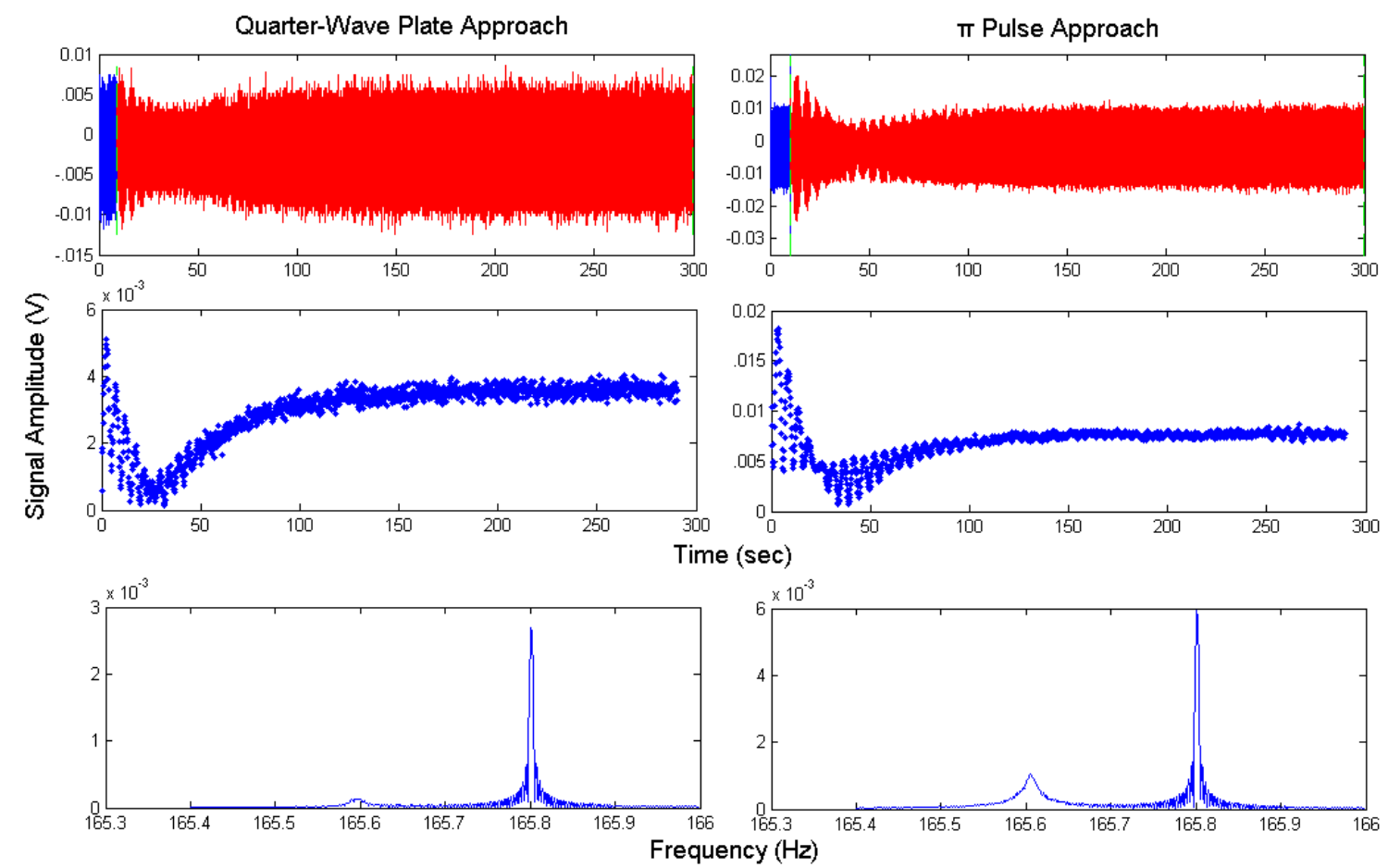

Figure 66. Raw data from both approaches to the flipped polarization method using a stimulating drive that was intentionally offset from the natural resonance frequency.

Notice the increased strength in the signal from the natural precession relative to the drive when using the pulse approach. This is due to the fact that the $\pi$ pulse is imperfect and almost always introduces some extra level of initial stimulation. For the plate approach, the $\mathrm{T} 2$ effects are so small in the profile that even with an arbitrary window size we can clearly see the $\mathrm{T} 1$ curve described by equation 6 , although it certainly carries some noise along with the beating. On the other hand, the pulse approach profile exhibits an entirely different shape in which the beating plays a severe role. Much like the on-resonance attempts at this method, the time at which the profile seems to hit zero occurs long after the half-life from the delayed pulse method. Interestingly though, the amplitude of the beating itself seems to come down to a minimum and then grow back up, and that minimum coincides with the expected half-life. 
In both cases, we use equation 8 to find the proper window size and construct our real data profiles along with the fit solutions using equation 6 , the details of which are presented in Table 9 and Figure 67 below.

Table 9. Summary of results comparing both approaches of the off-resonance flipped polarization (FP) method to the delayed pulse (DP) method.

\begin{tabular}{|c|c|c|c|c|}
\hline & FP Plate & FP Pulse & DP & Units \\
\hline $\boldsymbol{A}$ & 6.919296 & 13.92363 & 11.12021 & Volts \\
\hline $\boldsymbol{T 1}_{\text {fit }}$ & 36.4768 & 36.22239 & 28.77839 & Seconds \\
\hline $\boldsymbol{T 1}_{\boldsymbol{0}}$ & 35.66958 & 33.46045 & 33.98598 & Seconds \\
\hline $\boldsymbol{V}$ & 3.513126 & 7.339662 & 4.904676 & Volts \\
\hline $\boldsymbol{v}$ & 0.009721 & 0.255535 & 0.008523 & Volts \\
\hline
\end{tabular}
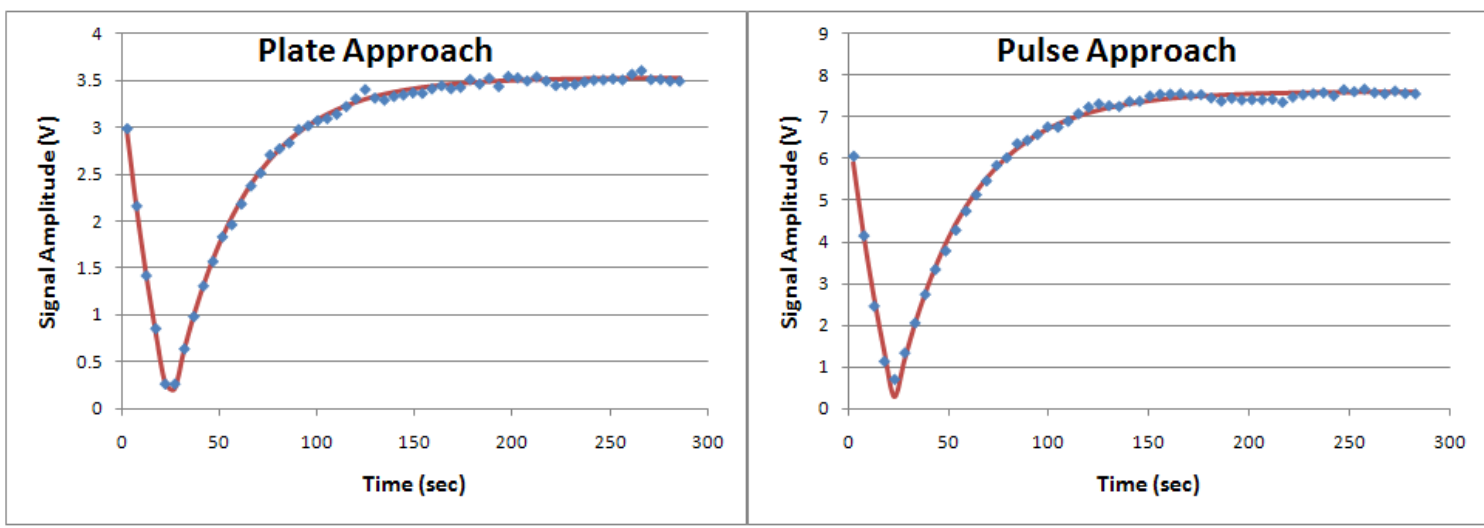

Figure 67. Signal profiles constructed from data in Figure 66 above, generated using the proper window size to eliminate dual-frequency beating and fitted with solutions using equation 6.

Much to our delight, the results from both tests match the delayed pulse $T 1_{0}$ value to within $10 \%$ ! They are also extremely similar to one another both in shape and zerocrossing. The results confirm that we can use either approach, so we usually choose the pulse approach given the ease of setup and the consistent data start time it provides. 
To get an idea of the level of consistency in this measurement, we repeat the pulse test ten times to find the statistics outlined in Table 10 below, displaying the mean and scatter associated with both $\mathrm{T} 1$ metrics as well as for the average of the two metrics for each run.

Table 10. Statistical results from ten repeated measurements of the ${ }^{129} \mathrm{Xe} \mathrm{T} 1 \mathrm{using}$ the off-resonance flipped polarization method.

\begin{tabular}{|c|c|c|c|}
\hline${ }^{\mathbf{1 2 9}} \mathbf{X e}$ & $\mathbf{T 1}_{\text {fit }}(\mathbf{s e c})$ & $\mathbf{T 1}_{\mathbf{0}}(\mathbf{s e c})$ & Average $(\mathbf{s e c})$ \\
\hline Statistical Mean & 32.24614605 & 31.930872 & 32.08851 \\
\hline Statistical Uncertainty & 0.16732932 & 0.45668 & 0.213586 \\
\hline
\end{tabular}

The $T l_{f i t}$ value seems to be the most precise, presumably because sometimes we get curves in which the initial and final amplitudes do not match. This tends to change the zero-crossing but not the overall shape of the curve, or in other words it seems to affect the relationship between the total amplitude $A$ of the signal and the vertical offset $V$ more than any other fit parameters in equation 6 . Even though $V$ seems to remain constant, if the ratio of $A / V$ strays far from a value of 2 , we will see significant changes in the time of the zero-crossing.

For some reason, however, when we use this method to measure the ${ }^{131} \mathrm{Xe} \mathrm{T} 1$ we see this effect occur more often and to a greater extent. It also does seem to affect the $T l_{f i t}$ value more noticeably for ${ }^{131} \mathrm{Xe}$. For example, compare the two fitted profiles below in Figure 68, whose solution parameters are given in Table 11. Both tests were performed for ${ }^{131} \mathrm{Xe}$ in the same cell under the same conditions, literally minutes apart from one another, and yet the results turned out quite different. 
Table 11. Comparison of solutions for differently shaped ${ }^{131}$ Xe T1FP curves shown below

\begin{tabular}{|c|c|c|c|}
\hline & Run 1 & Run 2 & Units \\
\hline $\boldsymbol{A}$ & 2.054513 & 1.366302 & Volts \\
\hline $\boldsymbol{T 1 _ { \text { fit } }}$ & 17.07681 & 23.41524 & Seconds \\
\hline $\boldsymbol{T 1 _ { \boldsymbol { 0 } }}$ & 25.34726 & 18.7411 & Seconds \\
\hline $\boldsymbol{V}$ & 0.734324 & 0.784527 & Volts \\
\hline $\boldsymbol{v}$ & 0.108032 & 0.079789 & Volts \\
\hline
\end{tabular}

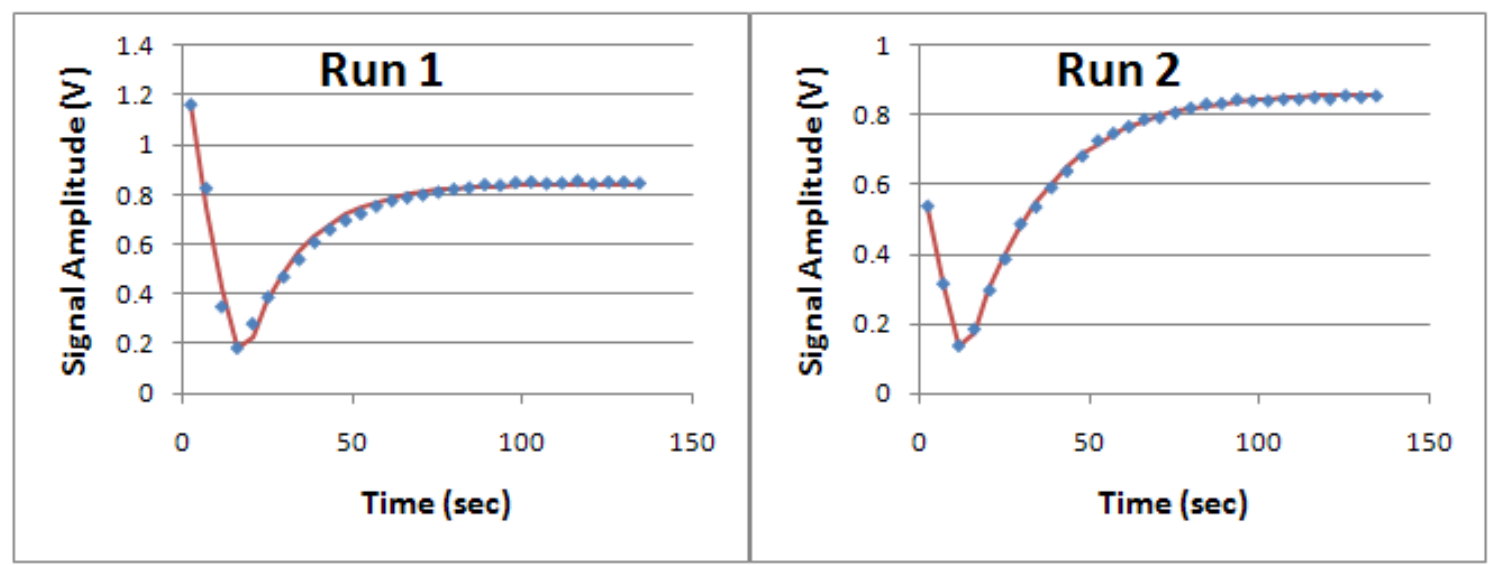

Figure 68. Comparison of differently shaped T1 flipped polarization curves for ${ }^{131} \mathrm{Xe}$.

Notice that when the initial amplitude is greater than the final amplitude, the $T 1_{0}$ value is greater than the $T 1_{f i t}$ value and vice versa. Also notice that there is quite a large discrepancy in the T1 metrics between the two data sets. The shape of the curve in Run 1 could be due to a $\pi$ pulse that introduced extra stimulation, but we would expect that to disappear with the T2 effects. The shape of Run 2 seems to indicate that the polarization had not fully recovered before the test was started, but we were careful to allow the same settling time between the data runs. When all is said and done, the statistics for ten ${ }^{131} \mathrm{Xe}$ flipped polarization tests are as listed in Table 12 below. 
Table 12. Statistics for ${ }^{131} \mathrm{Xe}$ off-resonance flipped polarization $\mathrm{T} 1$ results

\begin{tabular}{|c|c|c|c|}
\hline${ }^{{ }^{131} \mathbf{X e}}$ & $\mathbf{T 1}_{\text {fit }}(\mathbf{s e c})$ & $\mathbf{T 1}_{\mathbf{0}}(\mathbf{s e c})$ & Average $(\mathbf{s e c})$ \\
\hline Statistical Mean & 20.66189414 & 21.99977 & 21.33083 \\
\hline Statistical Error & 0.82087794 & 0.7528625 & 0.080835 \\
\hline
\end{tabular}

As we expected, given the strange inconsistencies in the shapes of the curves, the two T1 metrics have much larger error when used for ${ }^{131} X e$. Strangely, however, when taking the average of the two metrics we end up with an extremely consistent measurement of T1. It is unclear why the ${ }^{131} \mathrm{Xe}$ isotope exhibits less consistent behavior than ${ }^{129} \mathrm{Xe}$; it most likely has something to do with the quadrupole interactions with both the drive and the pulse simultaneously. We at least know that this technique has a measureable effect on the quadrupole population relative to the dipole population of ${ }^{131} \mathrm{Xe}$ atoms because the frequency-spectrum data from the flipped polarization method often differs from the FID FFT, as shown in Figure 69 below.

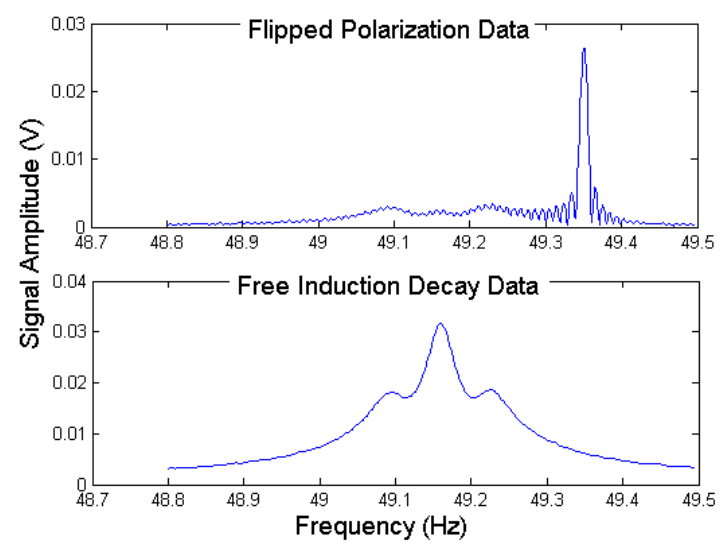

Figure 69. Comparison of FFT results for ${ }^{131}$ Xe using Flipped Polarization method and FID method. Notice that the quadrupole-induced side peaks seem to have the same frequency separation in both cases, but their amplitudes relative to the center peak are much higher in the case of the Flipped Polarization method. 
Although the frequency spacing seems to remain the same, the flipped polarization method seems to bring up the relative amplitudes of the side peaks to the point that they actually outweigh the central peak. This may explain the higher occurrence of strange shapes when measuring the ${ }^{131} \mathrm{Xe} \mathrm{T} 1$; since equation 8 is only capable of eliminating the T2 effects from the dipole population, quadrupole effects may still distort the curves. In any case, we at least seem able to produce reliable results using the average of the two metrics.

So, we have proven we can meet a pretty high level of accuracy with the flipped polarization method. However, if we want to replace our old method completely, we need to be confident that we can distinguish whether a given data run was successful or not. To do so, we must understand the sensitivities of the method and identify the most significant contributors to inaccurate results. As such, we want to explore some of the parameters responsible for shape defects even when using the off-resonance drive.

\section{d. Sensitivities in Flipped Polarization Results}

Experimentally, we suspect that the shape defects are caused by too large or too small a pulse, too high a drive frequency offset, insufficient settling time before the test, or a combination of these factors. On the analysis side, we wonder how precise our determination of the window size must be. 
We begin by trying the ${ }^{129} \mathrm{Xe}$ test over several pulse amplitudes ranging from a $\pi$ pulse down to nearly a $\pi / 2$ pulse. The results are actually quite interesting visually, so we present all of the curves below in Figure 70, labeled by pulse strength.

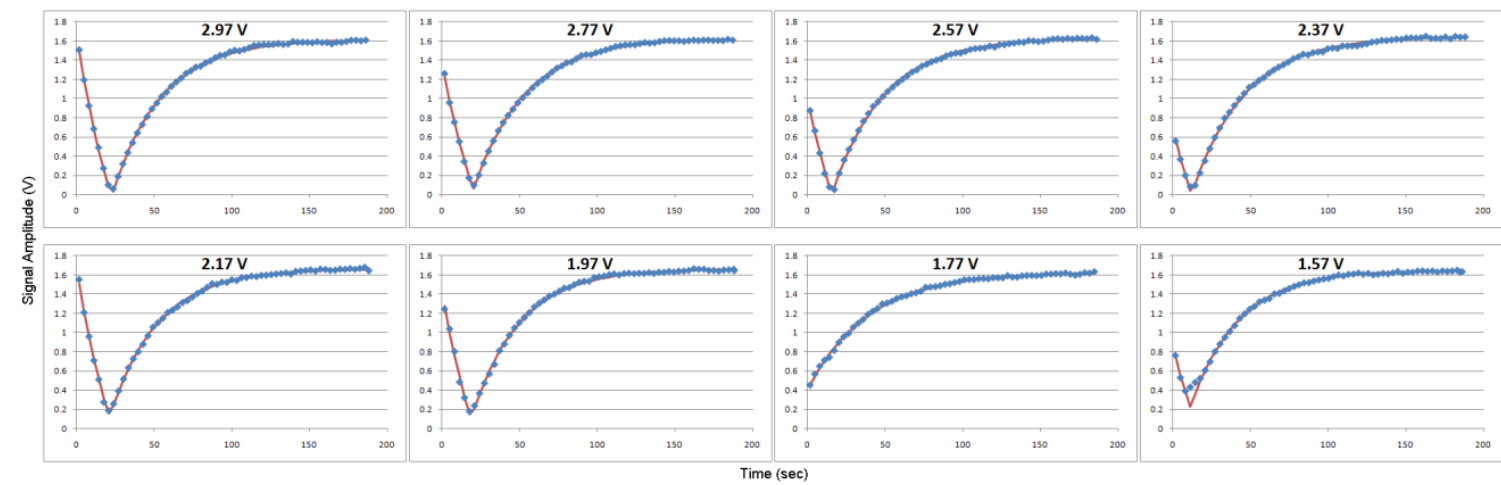

Figure 70. Flipped polarization curves obtained using various pulse strengths.

Notice that between $2.97 \mathrm{~V}$ and $2.37 \mathrm{~V}$, the initial and final amplitudes show greater discrepancies with smaller pulse strengths. Then, right around what would be a $3 \pi / 4$ pulse, the relationship between the two amplitudes seems to reset, although it definitely exhibits more extreme changes with the lower-strength pulses.

Of course, we also want to see the trend in fit solution parameters over this range of pulse strengths, so we plot the values of interest against pulse strength as shown in Figure 71 below. 

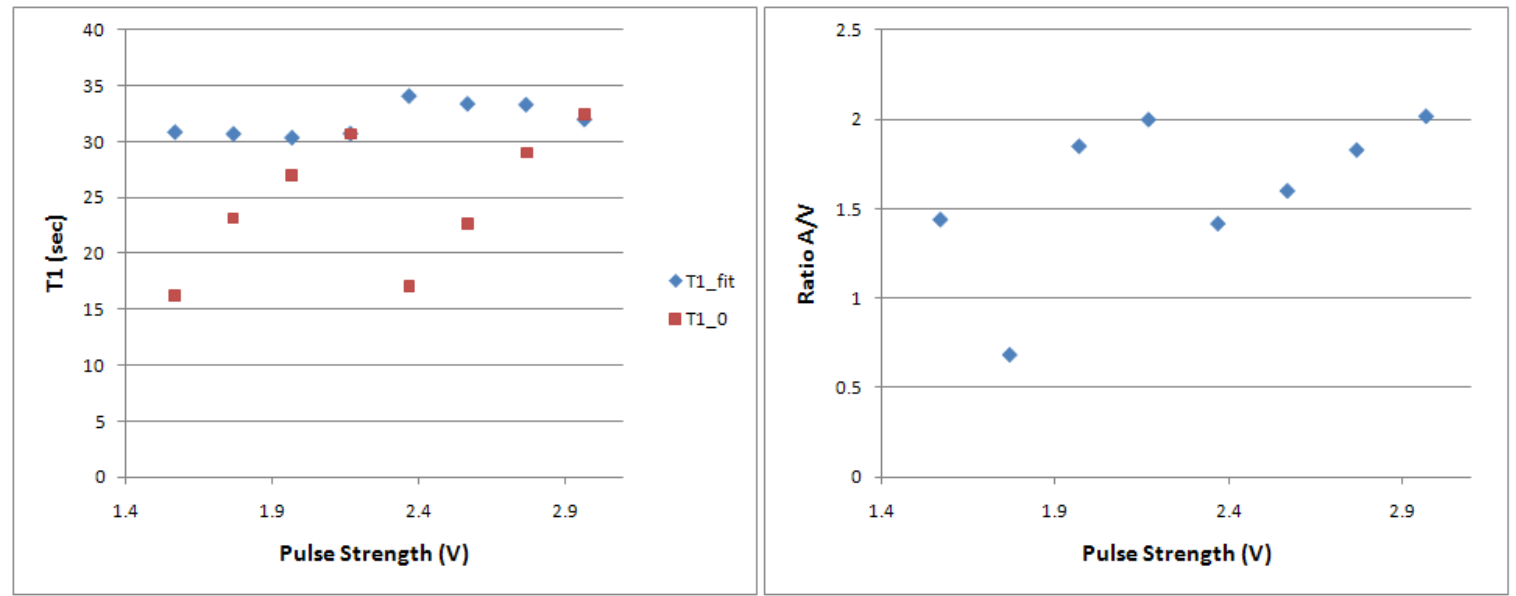

Figure 71. Relevant fit solution parameters plotted against pulse strength.

Again, we see a clear reset of the trends at the $2.17 \mathrm{~V}$ pulse mark. We find the most reliable results come from the tests in which the ratio of $A / V$ is close to 2 . With the exception of the amplitude ratio for the $1.77 \mathrm{~V}$ run, which probably had some external influence that was not accounted for, the two regions of pulse strength return extremely similar trends.

It is important to notice that the sensitivity to pulse strength varies greatly between the two T1 metrics. The $T 1_{\text {fit }}$ values only vary by about $5 \%$ of the maximum reported value, while the $T 1_{0}$ values vary by almost $50 \%$. For this reason, we tend to trust the $T 1_{f i t}$ value as the real measurement when using the flipped polarization method. So, for delayed pulse tests we use $T 1_{O}$ and for flipped polarization we use $T 1_{f i t}$. Either way, the two metrics should agree to high precision if the flipped polarization test is performed correctly, as indicated by the $2.97 \mathrm{~V}$ run. If that is not the case for a given run, we now know to repeat the test. 
We would generally expect the shape defects to become more prominent as we get farther off from a true $\pi$ pulse, so overall our predictions are correct. We would not expect a $3 \pi / 4$ pulse to return data that competes with the $\pi$ pulse for a clean shape, and we do not quite know how to explain why it does. Still, we have detailed the sensitivity to pulse strength for the flipped polarization method and proven the importance of using as true a $\pi$ pulse as possible.

Next, we would like to do the same investigation for drive frequency offset. We repeat the test several times with varying offsets, sweeping through the resonance frequency. The fitted curves are displayed below in Figure 72.
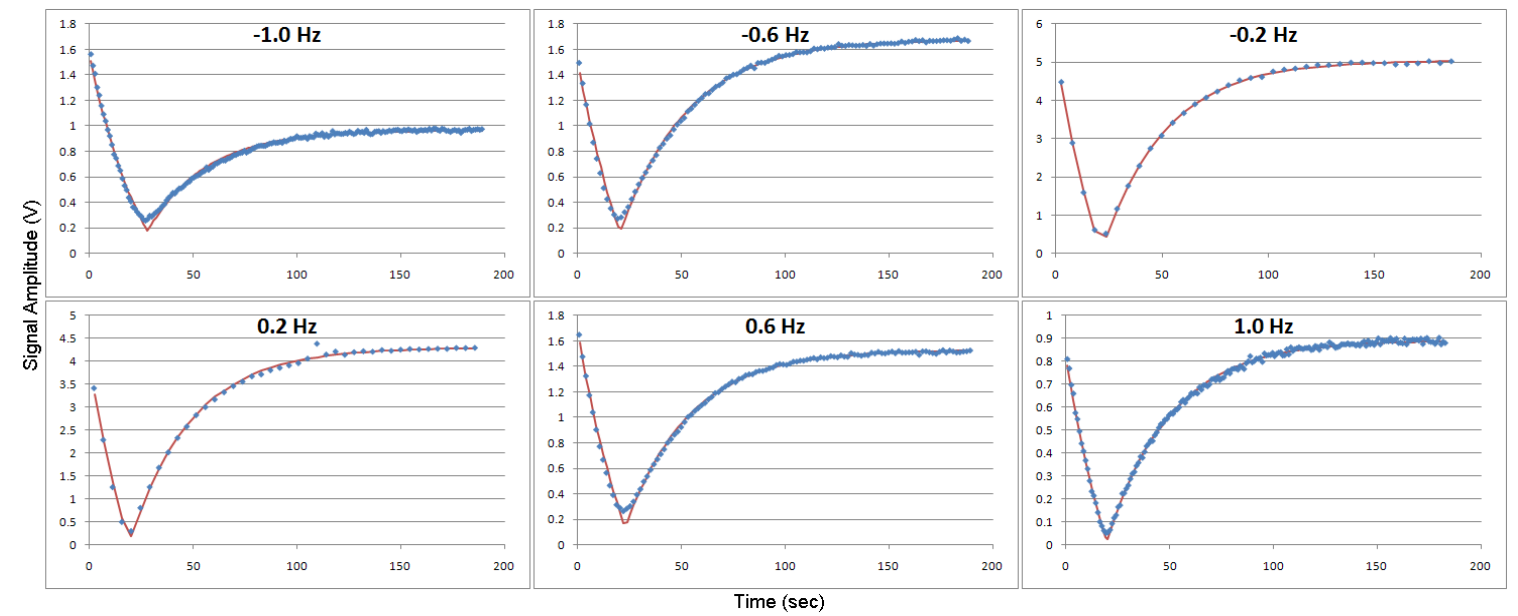

Figure 72. Flipped polarization curves using various levels of drive frequency offset.

The profiles all have fairly similar shapes with the exception of the $-1.0 \mathrm{~Hz}$ data run; the discrepancy between initial and final amplitude seems much less dependent on drive frequency than on pulse strength. However, there are some noticeable effects in the region of the zero crossing for all of the tests using greater than $0.5 \mathrm{~Hz}$ magnitude 
separation. These effects, as discussed previously, are evidence of poor cross-axis rejection; large frequency offsets result in very low signal amplitude and thus the $x$ drive actually comes through on the $y$ signal. Still, the cross-axis effects do not seem to hinder the fit function much.

Of course, just like with the T2 growth method, when we try to drive right on resonance we end up with some very strange effects, as shown in Figure 73.
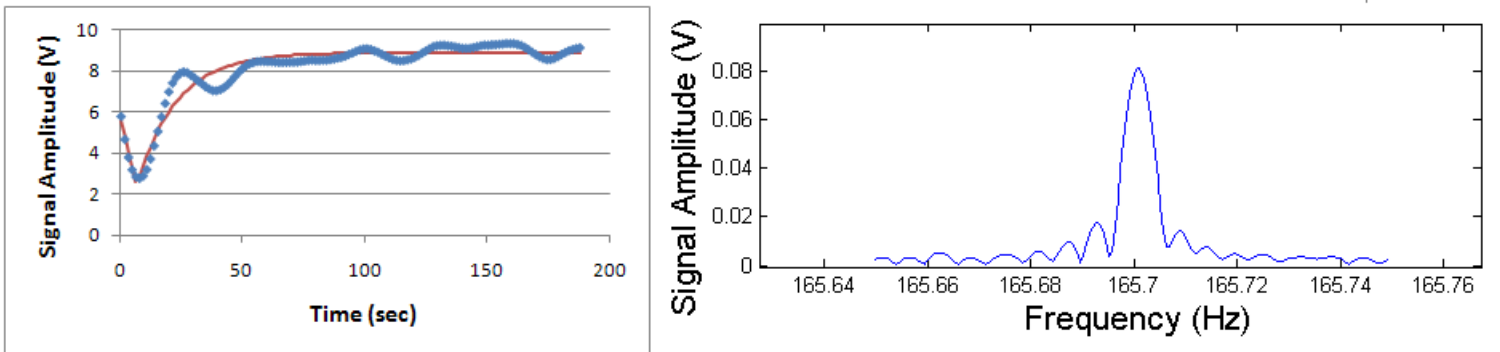

Figure 73. Data from a flipped polarization test using an on-resonance drive.

Ignoring the vastly different apparent half-life, which we already know to expect, the strangest part about the curve is that the beating occurs at a period of about 25 seconds, which should have a clear frequency separation of about $0.04 \mathrm{~Hz}$. Looking at the FFT, though, we see no such frequency separation, just the single distinct peak at resonance. The beating also seems to display some phase shifting throughout the run, especially apparent at about 76 seconds where there looks to be a sharp point in the curve. This complicates the issue even further. All we can really gather from this run is that we still know we cannot perform this test on resonance without incorporating the drive into our theoretical fit. 
Now we want to look at the trends in solution parameters over frequency offset, displayed below in Figure 74.

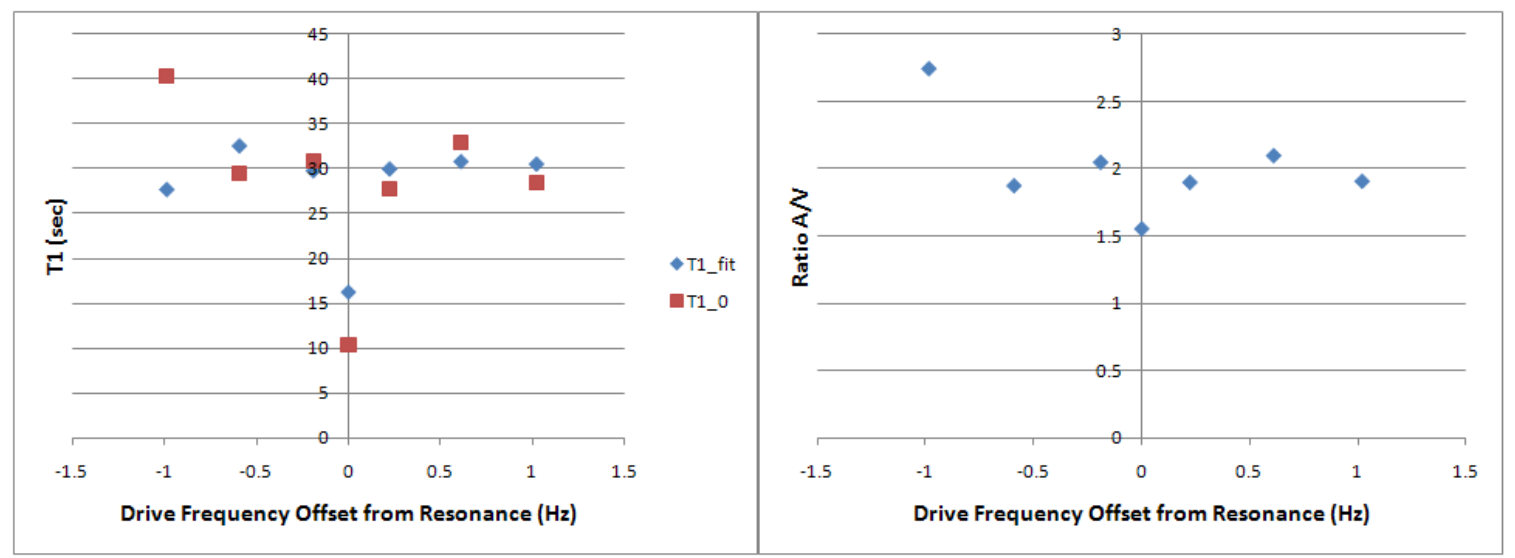

Figure 74. Sensitivities of the flipped polarization fit solution parameters to drive frequency offset.

Again, we see a clear correlation between the ratio of $A / V$ and the $T 1_{0}$ value, while the $T 1_{f i t}$ value seems to behave in the opposite way (increasing when $A / V$ decreases and viceversa). Both $\mathrm{T} 1$ metrics remain quite precise over the whole range of tested frequencies (with the obvious exception of the on-resonance case), but again $T 1_{f i t}$ is the clear winner in terms of consistency. This is highly valuable information because now we know we need not worry much about hitting any specific value for frequency offset when setting up this test. As long as we allow a large enough separation to yield the data point resolution we need, as governed by equation 8 , the test should work just fine.

As we discussed, we can also see shape defects if we do not allow sufficient settling time before starting a test. If such is the case, we expect to see a smaller initial amplitude than final amplitude since the test begins with some longitudinal spin cancellation already 
taking place. The data ends up looking like the beginning portion of the test gets cut off and everything else gets shifted backward to zero seconds. This problem can be avoided every time by simply waiting longer than needed; even if it does occur it should not affect the $T 1_{f i t}$ value. Also, it would be quite difficult to set up a repeatable experiment on this issue, so we will not spend time exploring it. We do, however, keep in mind that if ever a test turns out strangely and we cannot explain why, we should try repeating it with a longer settling time.

Now, there is one more factor that we expect can affect the results, and this one is part of the analysis process; what if our determination of the two frequencies is off and we use an incorrect window size? This is an easy problem to outline; we take the data from one of the previous tests that turned out well and alter the window size to see how it affects the results, as shown in Figure 75. The true window size should be 3.225 seconds. 

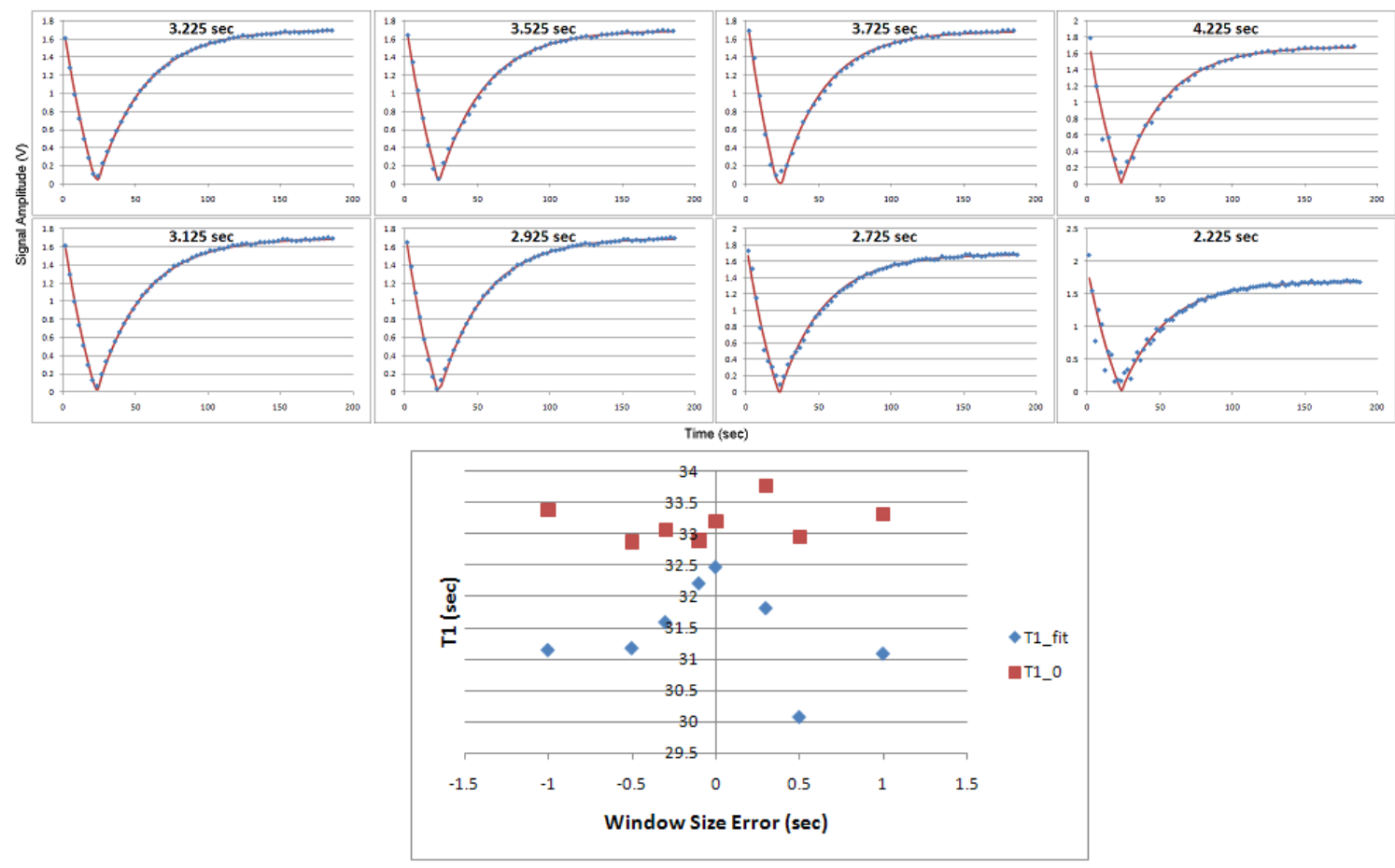

Figure 75. Fitted profiles and reported $\mathrm{T} 1$ values from varying window size for a single test run whose true window size should be 3.225 seconds.

Small errors in window size seem to have almost no effect on the profile shape unless the error is greater than 0.5 seconds in magnitude. Even then, the T1 metrics are both very consistent. We do see a larger discrepancy between the two T1 metrics with larger window size errors, but even if we go as far off as a whole second they never disagree by more than $10 \%$. If the window size was significantly off for a given test we would clearly see problems in the profile, like the high level of scatter in the 4.225- and 2.225-second cases, before the reported $\mathrm{T} 1$ values would become unreliable; plus, it is hard to believe we would ever be as far off as a whole second on the window size anyway.

So we have explored the major factors that we suspect would disrupt the flipped polarization test. We have determined that as long as the profile exhibits an $A / V$ ratio 
close to 2 and the two T1 metrics agree (say, within $10 \%$ of each other), the test was almost certainly performed successfully. At the very least, we can identify when something goes terribly wrong, and we can most likely determine the cause if so.

Now, we can finally argue with confidence that we can replace the delayed pulse method with the flipped polarization method as our primary T1 test. We can demonstrate the success of this test over a wide range cells; Table 13 below compiles the relevant information to compare the two methods over the most recent sample of our growing database and to assert the superiority of the flipped polarization method.

Table 13. Compiled results comparing the flipped polarization and delayed pulse methods

\begin{tabular}{|c|c|c|c|c|c|c|}
\hline $\begin{array}{c}\text { Cell } \\
\#\end{array}$ & $\begin{array}{l}\text { Inner Diameter } \\
(\mathrm{mm})\end{array}$ & $\begin{array}{c}\text { Cell } \\
\text { Geometry }\end{array}$ & $\begin{array}{c}\text { xenon } \\
\text { Isostope }\end{array}$ & $\begin{array}{l}\text { T1FP Error } \\
\text { (\%) }\end{array}$ & $\begin{array}{l}\text { Testing Time } \\
\text { Factor (FP/DP) }\end{array}$ & $\begin{array}{c}\text { Data Resolution } \\
\text { Factor (FP/DP) }\end{array}$ \\
\hline 1 & 1 & Cube & ${ }^{129} \mathrm{Xe}$ & 5.05 & 0.23 & 0.63 \\
\hline 2 & 2 & Cube & ${ }^{129} \mathrm{Xe}$ & 0.54 & 0.21 & 2.05 \\
\hline 3 & 2 & Cube & ${ }^{129} \mathrm{Xe}$ & 2.68 & 0.20 & 1.71 \\
\hline 4 & 2 & Cube & ${ }^{129} \mathrm{Xe}$ & 6.47 & 0.25 & 0.98 \\
\hline 5 & 2 & Cube & ${ }^{129} \mathrm{Xe}$ & 1.88 & 0.54 & 1.43 \\
\hline 6 & 4 & Cube & ${ }^{129} \mathrm{Xe}$ & 4.35 & 0.14 & 2.29 \\
\hline 7 & 4 & Cube & ${ }^{129} \mathrm{Xe}$ & 1.35 & 0.17 & 3.17 \\
\hline 8 & 8 & Sphere & ${ }^{129} \mathrm{Xe}$ & 0.09 & 0.20 & 3.65 \\
\hline 9 & 1 & Cube & ${ }^{131} \mathrm{Xe}$ & 0.00 & 0.20 & 0.52 \\
\hline 10 & 1 & Cube & ${ }^{131} \mathrm{Xe}$ & 5.94 & 0.21 & 0.41 \\
\hline 11 & 2 & Cube & ${ }^{131} \mathrm{Xe}$ & 4.11 & 0.38 & 1.02 \\
\hline 12 & 8 & Sphere & ${ }^{131} \mathrm{Xe}$ & 8.53 & 0.20 & 0.63 \\
\hline & & & Averages: & 3.42 & 0.24 & 1.54 \\
\hline
\end{tabular}

The T1FP Error column displays the error of the flipped polarization measurement for each cell, assuming the delayed pulse measurement to be the true value. The Testing Time Factor is the amount of time the T1 measurement took using the flipped polarization 
method divided by the amount of time it took using the delayed pulse method. The actual test times were first scaled by dividing each by its associated T1 value; otherwise, the settling time causes the delayed pulse method to take disproportionately longer for cells with high T1 times, and we do not want to artificially enhance the already impressive time reduction provided by the flipped polarization method. Finally, the Data Resolution Factor is a measure of the difference in data point density (number of points per unit time) between the two tests; it is generated by taking the number of data points divided by the length of time the data spans for each test, and then dividing that number for the flipped polarization run by the number for the delayed pulse run.

If we asked a Magic 8 Ball whether we should replace the delayed pulse method with the flipped polarization method as our primary T1 test, it should surely read, "All signs point to yes!" The new method is more than four times as fast, which means an average of about 30 minutes saved for every T1 measurement, producing results with $54 \%$ better data resolution and an average $\mathrm{T} 1$ error of only $3.42 \%$. Plus, we have the added bonus of statistical measurement if desired, which opens a completely new aspect of study for us. Finally, with such quick results we can more easily measure the T1 profile over temperature for every cell, which is important when studying the effects of cell wall properties.

Of course, there are still pitfalls of which to beware. First of all, the off-resonance drive puts significant SNR limitations on the method. The $\pi / 2$ pulse of the delayed pulse method already provides significantly higher signal than the on-resonance sustained 
drive; as we move off resonance the SNR difference widens quickly. Still, we have yet to encounter a cell where we could not achieve the signal necessary for a flipped polarization measurement but could for the delayed pulse. Presumably, this would only happen at relatively low temperatures where the magnetometer response is poor, or for cells with inherently low signal in which we would have little interest anyway.

The other main issue to remember is that the delayed pulse method essentially has a data resolution related to the amount of time taken to produce the data. On the other hand, the flipped polarization method has a data resolution related to signal strength since higher data point density requires a larger drive frequency offset. This means that for cells with very short lifetimes (under 10 seconds, say), it can be difficult to obtain a flipped polarization curve with sufficient data before the zero crossing to fit it reliably. However, when the $\mathrm{T} 1$ is that short, the time saved by the flipped polarization method is minimal, so we can make the exception to use the delayed pulse method in such a situation.

Overall, the flipped polarization method is a great success, and we intend to use it as the main $\mathrm{T} 1$ test henceforth. This means we have accomplished our goal to improve the lifetime test methods for NMR gyro cells, and quite authoritatively so! Still, there remains one more method we would like to explore - the Pump Growth Method - which could potentially measure both spin lifetimes in a single test run. 


\section{Measuring T2 and T1 Simultaneously Using the Pump Growth Method}

This last method is sort of a combination of the T2 growth and T1 flipped polarization methods. We are looking for a growth curve, but now the growth rate reflects both xenon spin lifetimes; although the setup is slightly more involved than the other methods, the opportunity to measure everything we need in one data run is quite tempting. Also, as with any new type of measurement, the results could reveal previously hidden information about the nature of the NMR test station.

The pump growth method is similar to the flipped polarization method in that our goal is to monitor the nuclear transition between longitudinal spin states while simultaneously stimulating the nuclear precession. The difference is that for this test the initial polarization state is the natural equilibrium. Instead of altering the pump direction, we want to monitor the atomic behavior from the instant that we first introduce the pump light at all.

Technically, the sense beam also performs some pumping, and although that is to a much lesser extent we may as well cut off all laser light through the cell when preparing the test. We usually do so by placing a solid block in front of each of the two lasers. We leave the magnetic fields and transverse drive running because they should have no measurable effect until the atoms are polarized by the pump beam. After enough time has passed with the blocks in place to allow any and all effects of the pump light to dissipate, we begin collecting data and pull up the laser blocks as quickly and synchronously as 
possible. Presumably, the alkali would be pumped very quickly and begin polarizing the xenon atoms as usual. At the same time, the transverse drive begins stimulating any xenon atom as soon as it is polarized. Unlike the flipped polarization method, in which the xenon stimulation is already at steady state by the start of the test, the pump growth exhibits $\mathrm{T} 2$ growth dependent upon $\mathrm{T} 1$ growth throughout the entire run. This complicates issues because, as we have seen, the $\mathrm{T} 2$ growth can behave in strange ways. Still, if everything goes as planned, we expect something like the process illustrated in Figure 76 below.

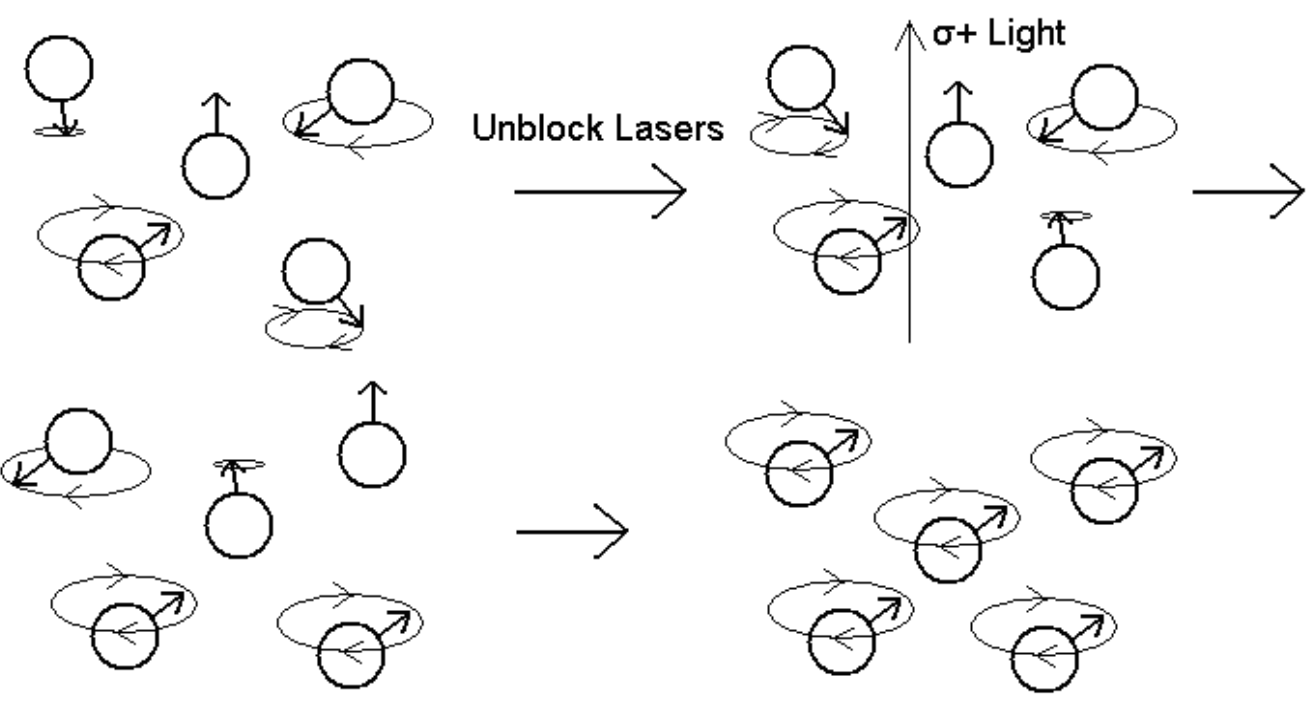

Figure 76. Visualization of the physical process monitored during the pump growth method. With the lasers blocked, there is no order to the polarization or the phase of precession among the xenon atoms. Once the lasers are unblocked, the atoms begin to get polarized by the alkali and stimulated by the drive until the steady state precession is reached.

Before the lasers are reintroduced, the xenon spin states are completely chaotic and should generate no gyro signal. By the end, we should see the signal generated by the 
steady state stimulation that we are used to. The process of the pump growth is the interesting part because, unlike any other method we have yet discussed, in this case the maximum level of overall stimulation is constantly changing throughout the run as the spin-up group becomes more and more populous. We expect to see a growth curve described by

$$
y=A *[1-\exp (-t / T 2)] *[1-\exp (-t / T 1)]+v
$$

since the T1 growth directly effects the amplitude of the T2 growth.

The general shape of this curve can change quite significantly based on the ratio of T2 to T1. Figure 77 below shows several different theoretical pump growth curves using various T2:T1 values.

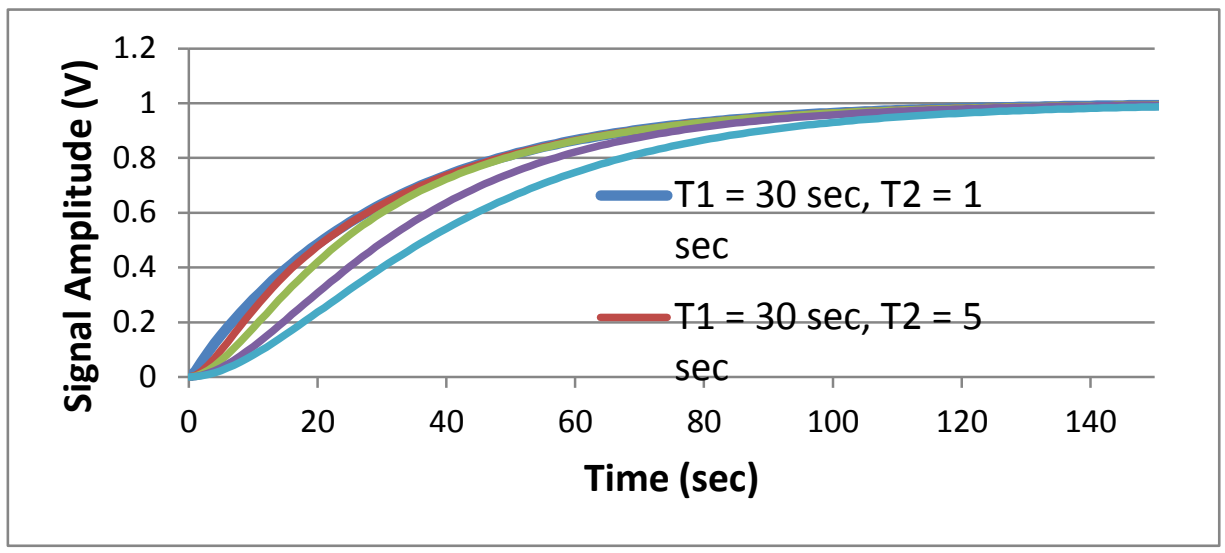

Figure 77. Theoretical pump growth curves using various ratios of T2:T1.

There is a clear difference in shape as the ratio of the two lifetimes changes. It makes sense that shorter $\mathrm{T} 2$ times would make the curve approach the final amplitude more 
quickly at the start of the run, as seems to be the case. The interesting part is the shift in concavity at the start of the run that emerges as T2 approaches T1. This gives us a sort of template with which to compare our actual data profiles, so that we can at least assess whether the shape of a profile seems correct given the lifetimes measured by the other methods.

Now, let's look at some real data; Figure 78 below shows the raw and processed data for a typical attempt at a pump growth test using the on-resonance drive for stimulation.
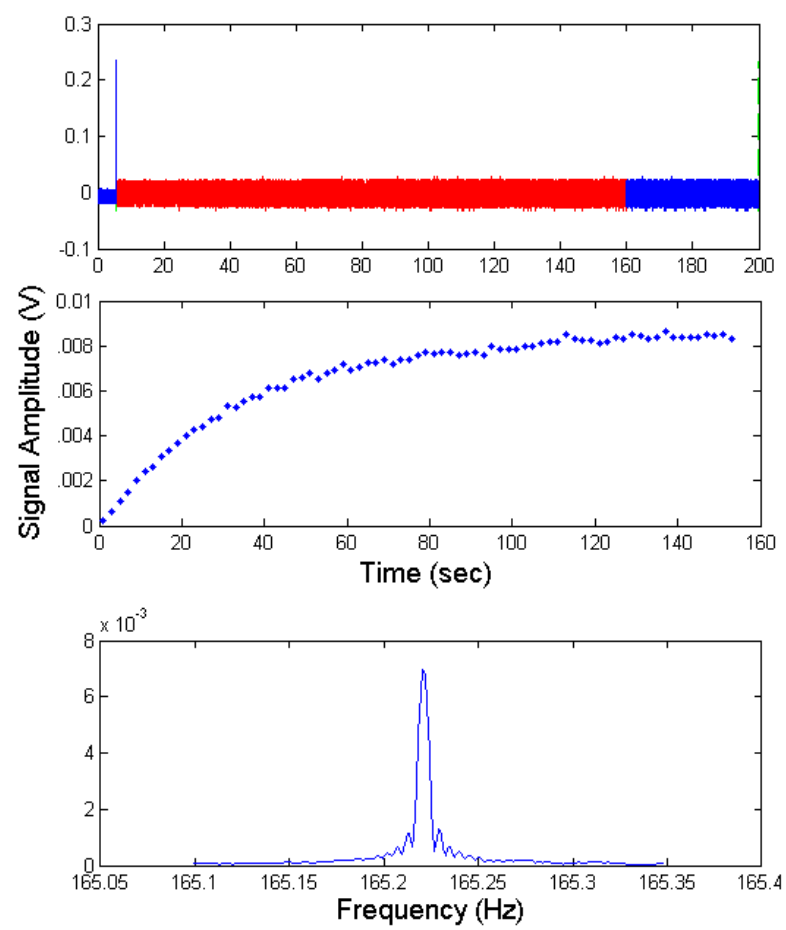

Figure 78. Example on-resonance-drive Pump Growth test data.

The time that the laser blocks were pulled up is indicated in the raw data by the one-sided spike in amplitude at about 5.6 seconds, which most likely comes from the electronics 
compensating for the large and fast change in light at the detectors. Fortunately, it shows up every time and allows us to identify the start of the test. Notice also that the apparent amplitude of the raw data shoots up immediately when the blocks are removed, presumably because the faraday detection picks up the scattered spins of the entire atomic population and effectively increases the noise level. However, the profile shows that the signal from the coherent precession of xenon atoms is effectively zero at the same instant, which hopefully means the test went well.

Now, the two lifetimes had been measured using the industry standard methods, as shown in Figure 79, reporting a T2 of 3.31 seconds and a T1 of 31.8 seconds.
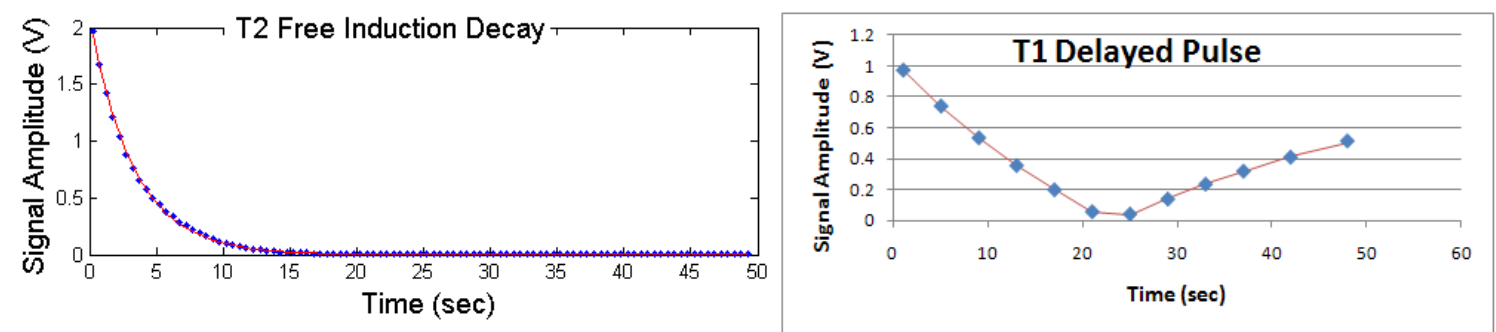

Figure 79. T2 and T1 measured by industry standard methods for the pump growth example cell.

So what do we find when we fit the profile from the pump growth test using equation 9 ? Figure 80 shows the fitted solution, which yields 3.77 seconds for T2 and 35.22 seconds for $\mathrm{T} 1$. So we have a $13.9 \%$ error in $\mathrm{T} 2$ and a $10.7 \%$ error in $\mathrm{T} 1$; the values are not perfect but they are not terribly far off, either. 


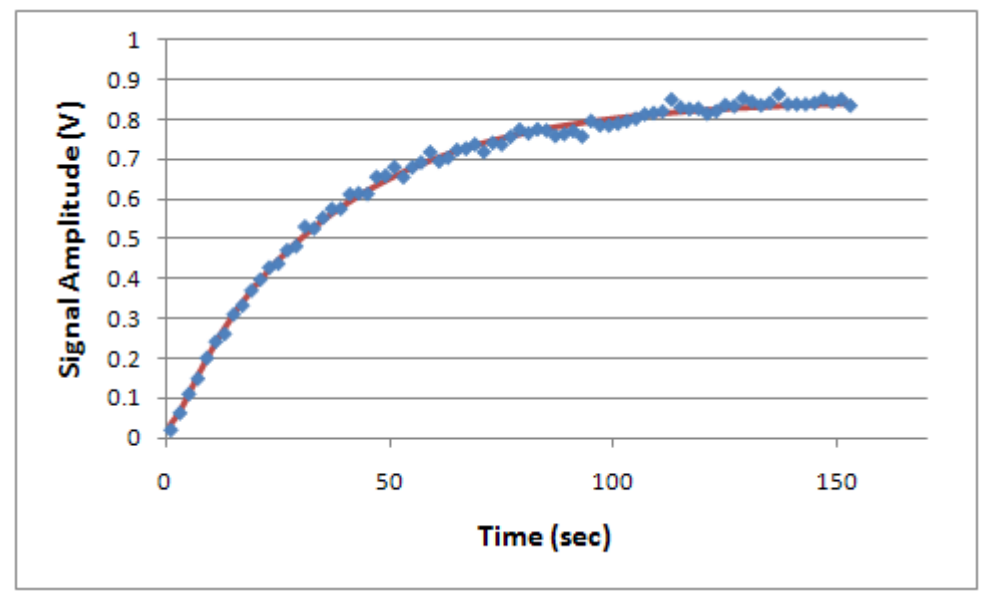

Figure 80. The fitted solution for the pump growth profile yields results that agree with the industry standard methods.

We could certainly make a case for this method if it worked this well every time, but of course it does not. This particular test turned out nicely because it was performed on an 8-mm-diameter spherical cell, rather large compared to our 2-mm and 1-mm cubes used as actual gyro cells. The larger size means more alkali vapor which means larger signal, which allows us to use a much weaker drive than we normally could, and unfortunately this method seems to be extremely sensitive to both drive amplitude and frequency, as shown in Figure 81 below. For the test over amplitude, the frequency was held close to resonance at $165.4 \mathrm{~Hz}$, and for the frequency test the drive amplitude was held at $1 \mathrm{mV}$. 


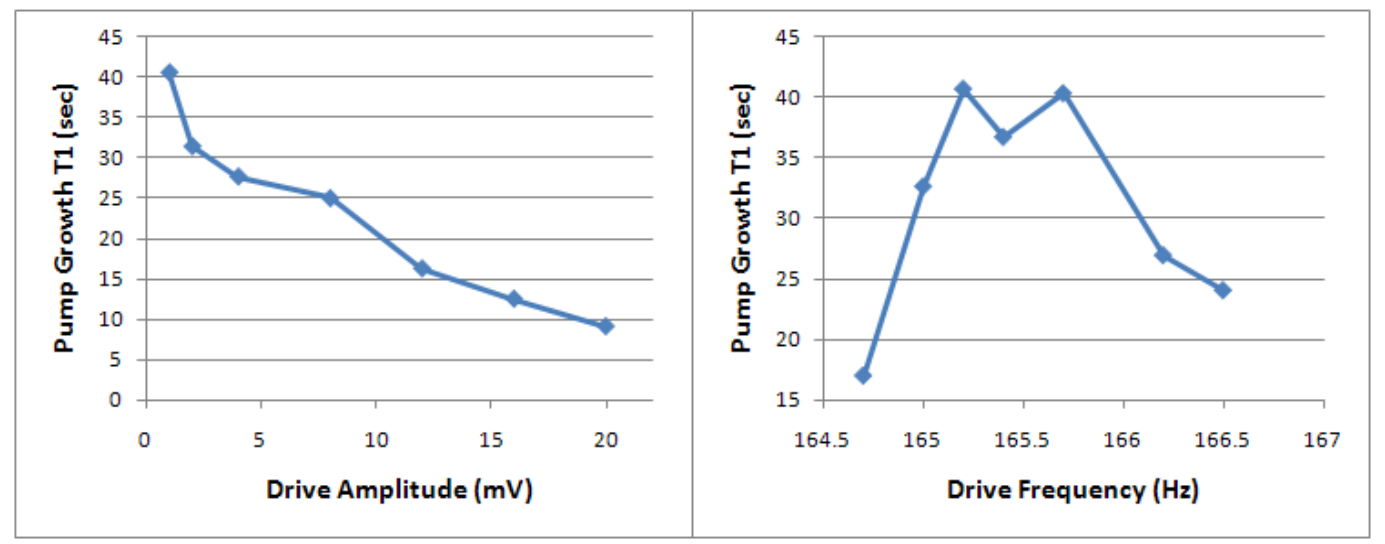

Figure 81. Pump growth T1 results over course range of drive parameters.

The $\mathrm{T} 2$ values in the fit consistently remain around 2 to 3 seconds; with the T1 hovering around ten times that value, the T2 barely affects the pump growth curve at all, so the T2 values are not displayed. The T1 results, on the other hand, show clear trends across the two drive parameters. The trend over drive amplitude sort of makes sense; as the drive amplitude increases we can convince ourselves that the overall stimulation process should speed up, though we would expect that to show up in the T2 rather than the T1. We would also expect to see some amplitude threshold below which the growth rate remains fairly constant, but we do not see such behavior.

The trend over drive frequency seems quite bizarre. Based on the amplitude behavior, we would expect to see the $\mathrm{T} 1$ increase as we move farther off resonance since that translates to lower effective drive amplitude. Perhaps that is the cause of the dip in the direct neighborhood of the resonance frequency; as we begin to move slightly off resonance we see the lifetime increase as the effective drive amplitude decreases. Then, some other unexplained effect takes over as we move too far off, which begins to drop the lifetime significantly. 
Whatever the reason, the values seem most similar to the delayed pulse results when the drive is very low and very close to resonance. Noting this, we want to repeat the tests with finer resolution in those regions. Unfortunately, our typical setup relies on an offthe-shelf function generator whose minimum output amplitude is $1.0 \mathrm{mV}$; for this test we had to insert an inline attenuator between the generator and the coils so that we could reduce the amplitude down to $0.1 \mathrm{mV}$. The results are presented in Figure 82 below.

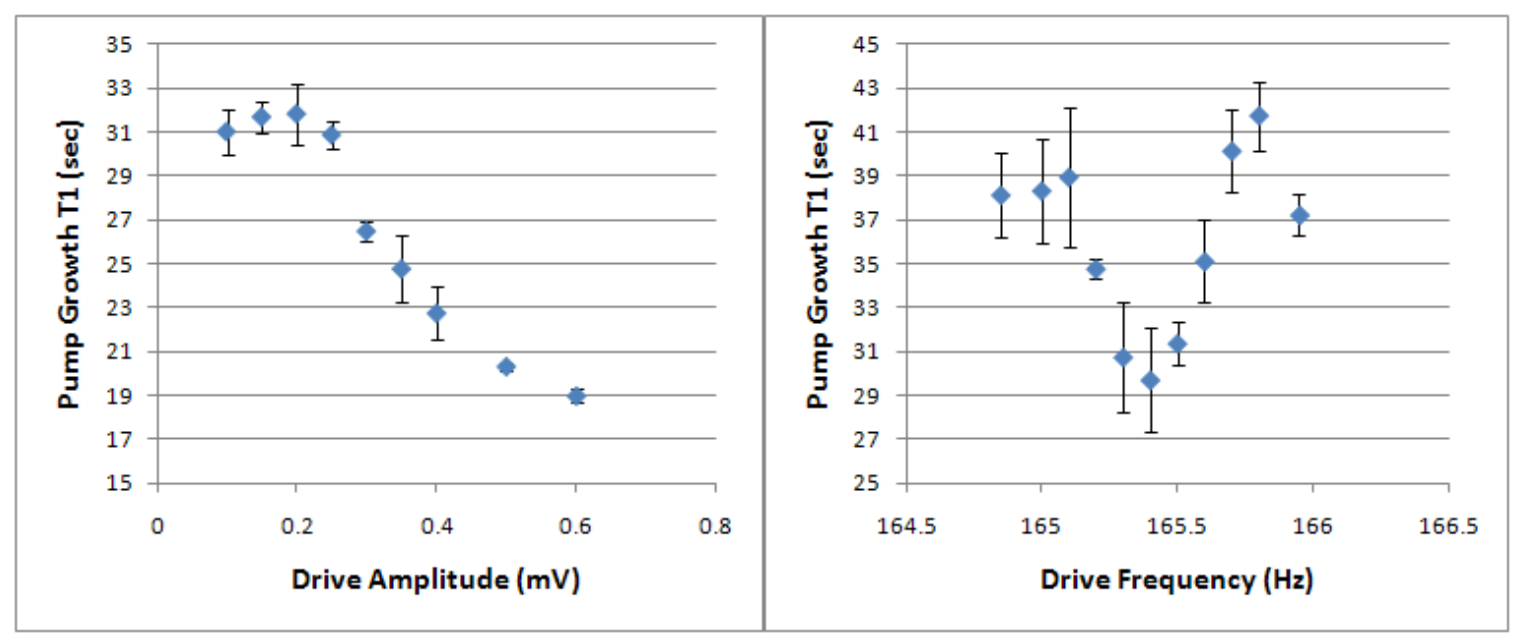

Figure 82. Pump Growth $\mathrm{T} 1$ results over fine range of drive parameters

To ensure that we are not simply seeing scatter disguised as a trend, we perform each run three times and fit each of the three curves five times. Each data point is an average of the three T1 values gathered from averaging the results from the five fits on each run. The error bars represent the statistical uncertainty for each point based on the standard deviation of the three $\mathrm{T} 1$ values for each case. Now, we can be quite certain that these effects are real. The reported $\mathrm{T} 1$ does indeed dip down at frequencies very near resonance, and presumably would again fall out on the wings of the frequency test. 
The strangest part is that the $\mathrm{T} 1$ follows almost the same trend over this narrow range of very low drive amplitudes that it did over the wide range of larger drive amplitudes in Figure 72 above. Perhaps there is a reset somewhere around 0.8 to $1.0 \mathrm{mV}$ for some reason, as we saw in the flipped polarization test over $\pi$ pulse amplitudes.

The good news is the consistency in $\mathrm{T} 1$ values with the drive between 0.1 and $0.3 \mathrm{mV}$, apparently the threshold we had hoped to see earlier. The bad news is that it ends at such a low drive amplitude, meaning we will never achieve strong signal with this method; most small gyro cells will not even produce a reliable curve at all with such little stimulation.

Still, all hope is not yet lost; we can attempt this method, as we have the others, using the intentional off-resonance drive. We take a new cell, a 2mm cube this time, with FID T2 measured at 21.9 seconds and FP T1 measured at 36.3 seconds. The natural resonance frequency was measured at around $165.63 \mathrm{~Hz}$. Below in Figure 83 is the data from an offresonance pump growth test driven at $165.8 \mathrm{~Hz}$ and $0.05 \mathrm{~V}$. 

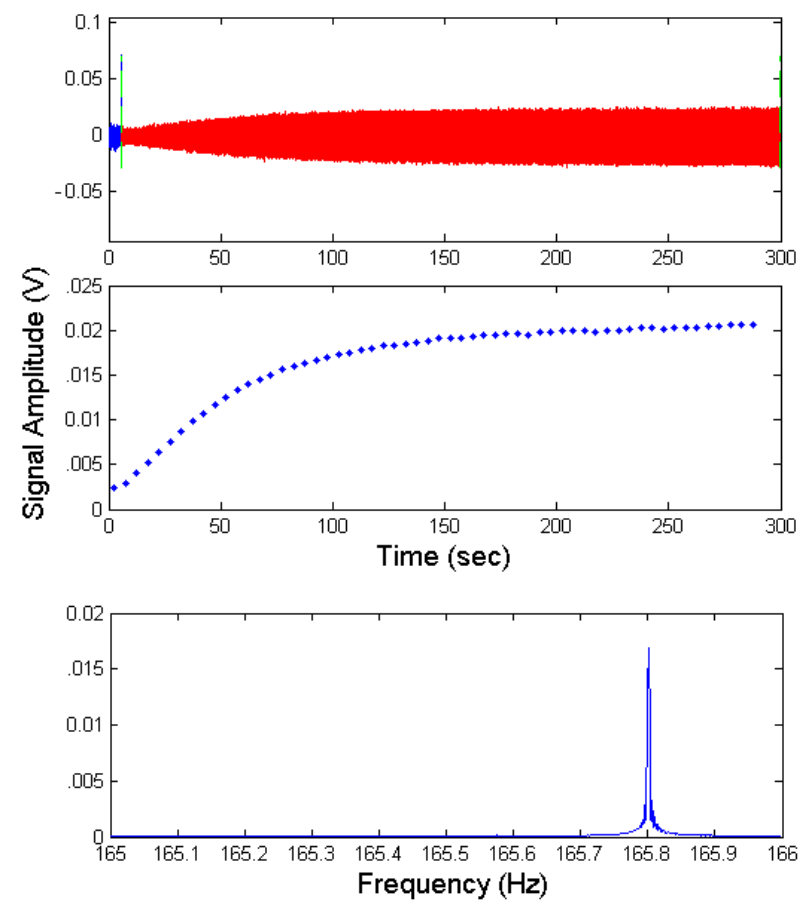

Figure 83. Data from off-resonance-stimulated pump growth test

Notice that the natural precession frequency is simply non-existent in the FFT data. Apparently, the pulse is solely responsible for stimulating the natural precession, so without said pulse there is no natural precession to be observed. It still seems like there should be some sign of the natural frequency since the $z$ field is still there and the atomic spins are offset from it, as we saw with the $\lambda / 4$ plate approach to the flipped polarization method, but the data here suggests otherwise.

Unfortunately, that also means that driving off resonance is not likely to help us since there is no beating to set the window size to; we are stuck with any and all drivedependent effects. To prove this, we repeat the off-resonance test at several different drive amplitudes, the results of which are shown below in Figure 84. 


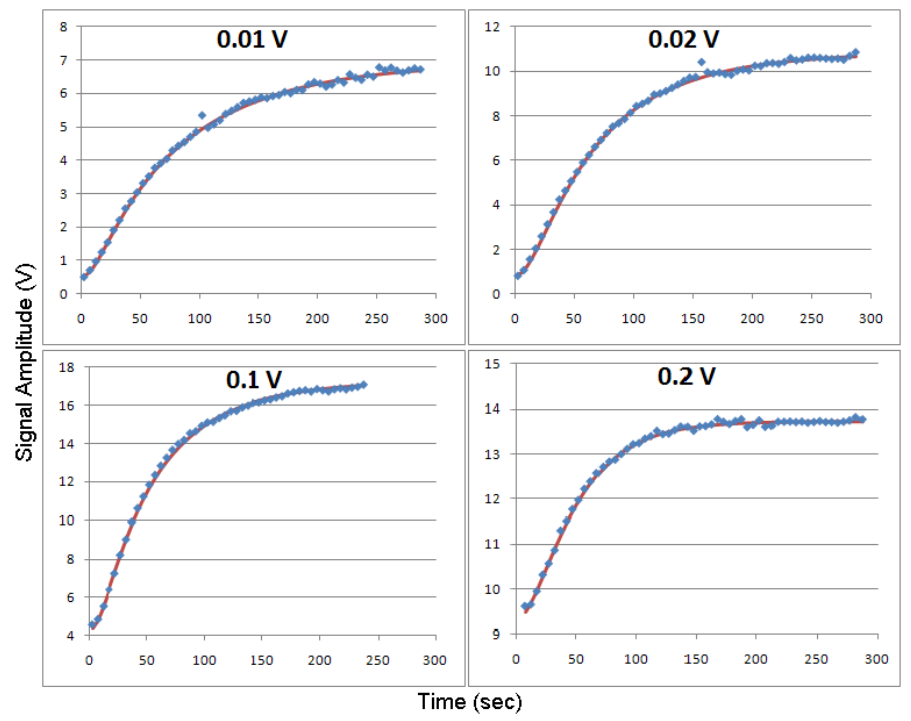

Figure 84. Off-resonance pump growth runs at various drive amplitudes

There is still a clear dependence on drive amplitude, even just by judging the shapes of the curves. We can certainly still get a good fit for each using equation 9, but the growth rate increases dramatically as the drive amplitude increases, and so does the vertical offset (most likely due to cross-axis pick up).

We can plot the $\mathrm{T} 1$ and $\mathrm{T} 2$ results from these tests over drive amplitude, as below in Figure 85 . Again, the T2 values remain fairly consistent and even accurate to the FID measurement to about $10 \%$. On the other hand, the T1 values are off by almost $300 \%$ at the lower-amplitude end. Oddly, the very high drive amplitude of $0.2 \mathrm{~V}$ yields quite an accurate $\mathrm{T} 1$ value, but a rather high $\mathrm{T} 2$ value as well. The $\mathrm{T} 1$ is most likely a coincidence, and even if not we find it difficult to justify relying on a curve whose vertical offset is nearly twice as large as the overall amplitude of the curve. 
In short, we cannot feel comfortable using a method that has so many inherent inconsistencies (or at best, many dependencies we do not yet understand).

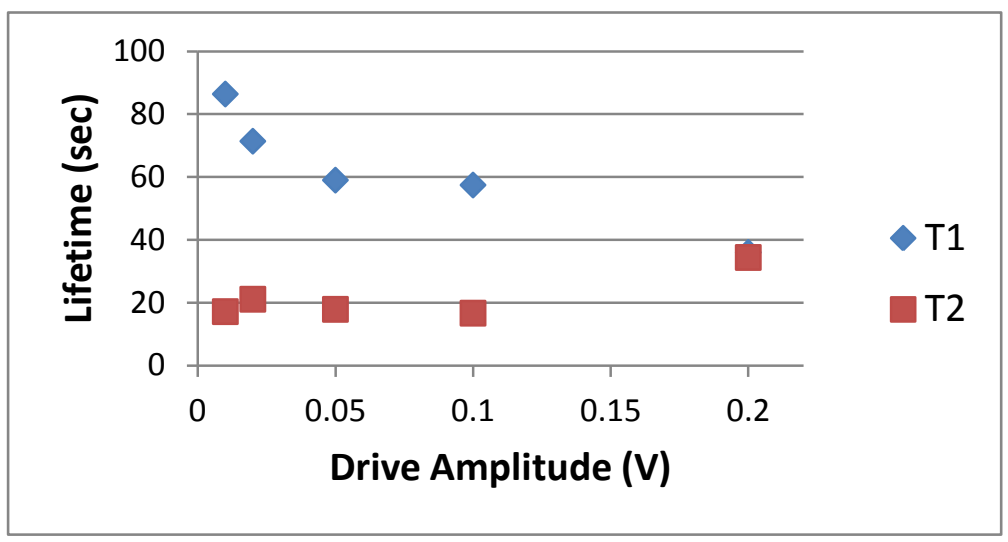

Figure 85. T1 and T2 results from off-resonance pump growth tests over drive amplitude

It seems strange that the shapes of the curves are so well described by equation 9 and that the $\mathrm{T} 2$ values are so accurate, even though the $\mathrm{T} 1$ values are so far off. There may be some valuable information to be gained by studying this method more closely; perhaps these curves reflect a real quality about the atomic system not visible by the other methods. More likely, however, it is merely a product of our inability to include the drive in our theoretical description of the curves, and so the fit function compensates for missing components by skewing the T1 value. In either case, the solution lies beyond the scope of our current investigation.

As it stands, we know the pump growth method can work because we have seen it from time to time, but the conditions need to be ever so perfect that we have no reason to prefer this method. With that said, it will be important to continue to study the pump growth behavior because it obviously exhibits effects that we do not yet understand. We 
may be able to gather valuable information about the atomic pumping process in our system by developing the means to accurately describe the pump growth curves. For now, though, it shall remain an exercise for the future. 


\section{Conclusions}

We opened our discussion with the basic motivation of improving navigation sensors to support the future of aerospace technology, and eventually we narrowed our focus to a very specific type of sensor - the NMR gyro - which is still in the research phases of development but has the potential to dominate the gyroscope industry in the next decade or two. After building up a cursory understanding of the operation of the device, starting with the basic atomic physics behind magnetic resonance in general, we centered our discussion on identifying the main parameters that affect NMR gyro capabilities and implementing the common techniques to test those parameters.

We found that the free induction decay method, the industry standard technique for measuring transverse spin coherence lifetimes in our test system, is extremely precise, with statistical errors on the order of 10 milliseconds for lifetimes in the 10 to 30 second range. We also explored the complications involved when working with the electricquadrupole-sensitive ${ }^{131} \mathrm{Xe}$ atom and established ways to effectively neutralize such complications, giving us reliable means to test the coherence of both xenon isotopes crucial to the gyro system.

Our other industry-standard technique, the delayed pulse method for measuring longitudinal spin lifetimes, we found very slow to produce results; a typical test takes about 40 minutes, and even longer tests are not uncommon. However, we outlined ways to minimize the time required by collecting only the necessary data points. Also, by 
establishing a standard fit function, we provided the means to extract more consistent results from this test than was previously possible.

The growth methods, both the stimulated growth for measuring T2 and the pump growth for measuring the two lifetimes simultaneously, were mostly unsuccessful. Although we found clear evidence that the growth tests can be made to yield accurate results, the difficulty of setup, sensitivity to drive parameters, and inconsistency of results associated with these methods convinced us to seek better options.

Finally, after months of frustration, the flipped polarization method for measuring T1 proved to be highly successful and, in fact, quite superior to the industry-standard delayed pulse technique for several reasons. Primarily, the new method cuts down the time required to make a $\mathrm{T} 1$ measurement by over $75 \%$ on average, saving us as much as 30 minutes on each cell we test, while yielding results within $3.5 \%$ of the industrystandard measurements. It is generally insensitive to drive amplitude and drive frequency (as long as the frequency offset is sufficient to provide the data resolution necessary), and we were able to outline the other major sensitivities to help guarantee successful implementation on a consistent basis. The statistical error for the new method is in the range of 100 to 200 milliseconds, well within our tolerance, and even just the ability to provide statistical data gives the flipped polarization a huge advantage over the delayed pulse because it would literally take days to gather the data necessary to make a comparable statistical assessment of the industry-standard technique. As rare an 
occurrence as it is, there truly seems to be no downside to the new method, unless you count the fact that we spent nearly a year attempting to implement it successfully. If time and money permit, we could develop automated loops for these and similar cell tests to build up our assessment of cell trends and of our system as a whole as quickly as possible. As we continue to gather new data more quickly, we could develop better software capabilities to automate the test procedures and to extract the relevant numbers from batches of files. Much of the analysis software along these lines has been developed in house, but further tailoring would certainly help debug the code and amplify the effectiveness of the work presented here.

We have little doubt that NMR-based sensor technology will flourish over the next few decades, and likely it will lead the market for certain applications. However, vapor cell design remains one of the major obstacles for NMR product development due to the level of inconsistency associated with cell production; by expediting the cell test procedure, we have not only provided the means to locate gyro-grade cells faster in a production line scenario, but have also opened the door for better research in the area of cell design. With the promise of faster results, more widespread cell filling and gas mixing techniques can be explored, which should lead to greater capabilities in NMR cell manufacturing. Plus, now with the added ability to measure gas pressures after sealing the cells, we can easily correlate cell performance improvements to specific cell manufacturing processes to help optimize the filling and sealing methods. 
As the field of study matures and NMR starts becoming a standard for sensor systems, it will be interesting to see just how far the technology can be pushed. Personally, I look forward to the day that the first commercial NMR gyro makes its way into orbit, and hopefully we'll be able to trace some of its successes back to our work here. If nothing else, I hope this dissertation has brought readers to the level of fascination in which I found myself when I began my studies in NMR. There is much to gain from learning about a cutting-edge science, and even more so when given the opportunity to be a part of its development. For this I am grateful, and I hope to contribute much more in the years to come. 


\section{REFERENCES}

${ }^{1}$ Barbour, Dr. Neil. Navigation: What happens inside a spinning gyroscope? Access Science. The McGraw-Hill Companies. April 22, 2004. Image credit: Dr. Neil Barbour and The Charles Stark Draper Laboratory, Inc.

${ }^{2}$ Griffiths, David J. Introduction to Quantum Mechanics. Upper Saddle River, NJ: Pearson Prentice Hall, 2005.

${ }^{3}$ Saleh, Bahaa E. A. and Teich, Malvin Carl. Fundamentals of Photonics. USA: John Wiley \& Sons, Inc., 1991.

${ }^{4}$ Einstein, Albert. On the Quantum Theory of Radiation, Received March 3, 1917. Published by Van Der Waerden, B. L. in Sources of Quantum Mechanics. Ontario, Canada: General Publishing Company, Ltd., 1968.

${ }^{5}$ Happer, Jau, and Walker. Optically Pumped Atoms. Federal Republic of Germany: Wiley-VCH, 2010.

${ }^{6}$ Greenwood and Simpson. Fundamental Noise Limitations in Magnetic Resonance Gyroscopes. Little Falls, NJ: The Singer Company, Kearfott Division, 1977.

${ }^{7}$ Kwon, Mark, and Volk. Quadrupole Nuclear Spin Relaxation of ${ }^{131}$ Xe in the Presence of Rubidium Vapor. Physical Review A: The American Physical Society, October 1981.

${ }^{8}$ Slichter, Charles P., Ph. D. Principles of Magnetic Resonance. Springer-Verlag, Germany: 1992 